\begin{abstract}
Duke, Kelly Scott. Productivity and Ergonomic Investigation of Bent-Handle Pliers. (Under the direction of Dr. Gary A. Mirka.)

Musculoskeletal disorders (MSDs) cost industry billions of dollars in workers' compensation costs each year. Ergonomics is concerned with understanding the causes of MSDs and developing solutions to reduce these costs. Awkward postures have been implicated as a risk factor for the development of some MSDs, and a design principle to "bend the tool, not the wrist" has been advocated in many ergonomic textbooks. However, despite numerous laboratory investigations showing positive outcomes of application of this design principle to various hand tools, there is indication of lack of acceptance in industry for these bent-handle tools. In an attempt to understand the lack of industry acceptance, this investigation sought to determine if this design principle imposed constraints on users and/or negatively affected productivity, which may explain why they are not being widely used in industry.

The experiment used two different tasks (a computer-jumper installation task, and a spring assembly task) to compare the use of bent-handle pliers versus straight-handle pliers. Additionally, the effect of work surface orientation (vertical versus slanted at $45^{\circ}$ ) was evaluated, as was the effect of constraining the user's coupling of the tool. The dependent variables in the experiment were productivity and postural outcomes (arm elevation, wrist deviation in the radial/ulnar plane, and wrist deviation in flexion/extension).
\end{abstract}

An important point that must be made is that overall the results clearly suggested that the expected outcomes (both productivity and postural) are very task specific. This in 
itself says a lot about the general recommendation to "bend the tool", that being that the recommendation cannot be made without clearly understanding the other task characteristics involved, and that it should therefore not be proposed as a general design recommendation.

For the computer-jumper task the bent-handle pliers resulted in $5.3 \%$ faster task performance compared to the straight-handle pliers, while for the spring assembly task the performance was $4.9 \%$ faster with the straight-handle pliers. The explanation provided is that the bent-handle pliers seem to be preferable for tasks that require minimal or no tool rotation out of the sagittal plane, losing their advantage when multiplane rotation is required. When subjects were constrained to holding the pliers with a power grip or oblique grip (modified power grip) arm elevation was reduced $50 \%$ and ulnar deviation was reduced by $12 \%$ when using the bent-handle pliers on the computerjumper task, while on the spring assembly task ulnar deviation was reduced $22 \%$. These results suggest that there are postural advantages to the bent-handle pliers (for the tasks used in this experiment) when the pliers-coupling is restricted to these grips. In the test of constrained versus unconstrained grip the results showed that for the computer-jumper task the postural advantage of the bent-handle pliers over the straight-handle pliers was lost when the method of gripping was left to the subjects. In addition to showing that postural benefits may only be seen when the pliers are held in a specific way, these results (along with others discussed in the paper) illustrate that expectations associated with this design concept are very task-specific. Finally, removal of the coupling constraint also showed that subjects were more likely to grip straight-handle pliers in alternative ways compared to the bent-handle pliers, which were more likely to be held 
with a power or oblique grip, even when not constrained. In summary, it appears that the specificity of the bent-handle tool design, may make it better in specific circumstances, but if the use requires increasing degrees of manipulation, it is less likely to be superior to a simpler design. 


\title{
Productivity and Ergonomic Investigation of Bent-Handle Pliers.
}

by

\author{
Kelly Scott Duke
}

A thesis submitted to the Graduate Faculty of North Carolina State University in partial fulfillment of the requirements for the Degree of Master's of Science.

INDUSTRIAL ENGINEERING

Raleigh, North Carolina

June 2002

Approved by:

Chairperson of Advisory Committee 


\section{BIOGRAPHY}

Kelly Scott Duke was born in Calgary Canada in 1970 to Paul and Carol Duke. He has three older brothers, Steven, Marty, and Robert. He lived, and loved living, in Calgary throughout his childhood, adolescence, and into adulthood - leaving in 1993 to move to Vancouver, British Columbia to attend Simon Fraser University. At SFU he attained a Bachelor's of Science degree in Kinesiology in 1996.

Travel and adventure have played an important role in Kelly's life. Before attending SFU, Kelly backpacked around the South Pacific, going to the Cook Islands, Fiji, New Zealand, and Australia. After SFU, Kelly backpacked around Central America, going to Costa Rica, Panama, Nicaragua, Honduras, Guatemala, and Mexico.

Kelly moved to "the South" in 2000 to attain a Master's of Science in Industrial Engineering. At North Carolina State University he worked as a Research Assistant to Dr. Mirka, while taking classes in Industrial Engineering (Ergonomics) and Psychology (Human Factors). 


\section{TABLE OF CONTENTS}

LIST OF TABLES............................................................... vi

LIST OF FIGURES............. vii

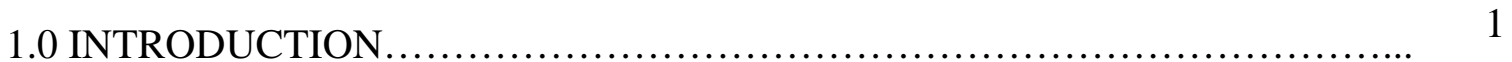

1.1 Cost to Business............................................................ 1

1.2 Causes of Musculoskeletal Disorders................................. 1

1.2.1 Posture as a Physical Risk Factor..................................... 3

1.3 Implications for Hand-Tool Design................................... 5

1.3.1 "Bend the Tool"............................................... 5

1.3.2 Previous Research on Bent-Handle Tools.......................... 5

1.3.2.1 Hammers........................................... 5

1.3.2.2 Files.................................................. 8

1.3.2.3 Knives............................................. 9

1.3.2.4 Pliers.................................................... 10

2.0 BENT-HANDLE TOOLS IN INDUSTRY ................................. 14

2.1 Proposed Rationale for Lack of Acceptance............................... 14

2.2 Hypotheses...................................................... 15

3.0 MATERIALS AND METHODS.......................................... 16

3.1 Experimental Tasks................................................. 16

3.1.1 Jumper Task................................................... 16

3.1.2 Spring Task.................................................... 17

3.2 Apparatus............................................................. 19

3.3 Independent Variables............................................. 20

3.3.1 Pliers........................................................ 20

3.3.2 Constraint................................................. 21

3.3.3 Work Surface Orientation..................................... 21

3.4 Dependent Variables............................................... 22

3.4.1 Productivity ..................................................... 22

3.4 .2 Posture ...................................................... 22

3.4.2.1 Wrist............................................. 22

iii 
3.4.2.2 Shoulder.......................................... 22

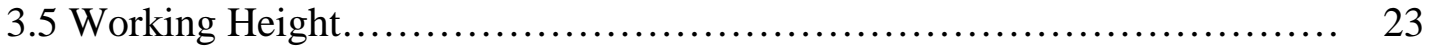

3.6 Subjects........................................................... 24

3.7 Protocol............................................................ 25

3.7.1 Anthropometry ............................................ 25

3.7.2 Instrumentation............................................ 25

3.7.3 Neutral File Collection........................................ 25

3.7.4 Trial Randomization........................................... 27

3.7.5 Subject Instructions.......................................... 27

3.7.6 Subject Practice ............................................... 27

3.7.7 Trial Data Collection............................................. 28

3.8 Data Analysis....................................................... 29

3.8.1 Data Processing ............................................... 29

3.8.1.1 Productivity ........................................... 29

3.8.1.2 Wrist Posture....................................... 29

3.8.1.3 Shoulder Posture....................................... $\quad 30$

3.8.2 Statistical Analysis......................................... 30

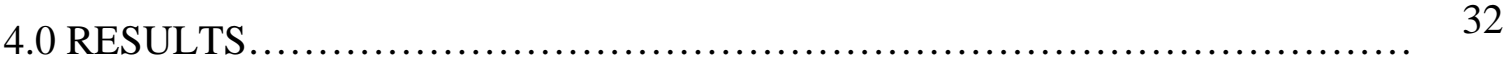

4.1 Tests of Assumptions................................................ 32

4.1.1 Test for Assumption of Normal Distribution of Residuals........... 32

4.1.1.1 Jumper Task........................................ 32

4.1.1.2 Spring Task......................................... 37

4.1.2 Test for Assumption of Equal Variance.............................. 41

4.1.2.1 Jumper Task........................................... 41

4.1.2.2 Spring Task......................................... 45

4.2 ANOVA Results................................................... 50

4.2.1 Jumper Task............................................... 50

4.2.2 Spring Task ............................................... 59

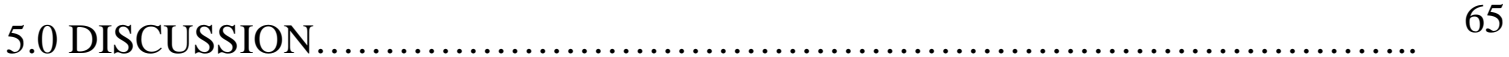

5.1 Results Directly Addressing Hypotheses............................... 65

5.2 Other Significant Results........................................... 68 
5.2.1 Flexion/Extension............................................... 68



5.2.3 Arm Elevation (Shoulder Posture) ................................ 69

5.2.4 Radial/Ulnar Deviation.................................... $\quad 70$

5.3 Further Discussion Regarding Tool Coupling............................ 70

5.4 Relating This Study to Previous Works................................. 74

5.5 Future Research...................................................... 76

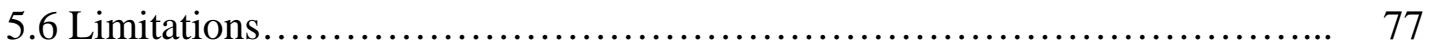

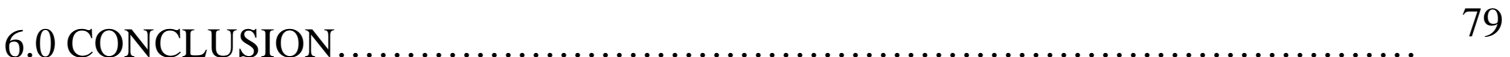

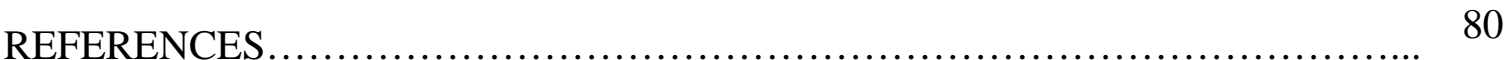

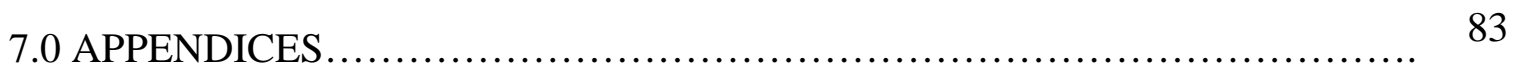

7.1 Work Surface Height Determination Data.............................. 83

7.2 Sample Data Collection............................................. 85

7.3 Informed Consent Form.............................................. 86 


\section{LIST OF TABLES}

Table 3.1: Work Surface Heights for both Tasks by Orientation................... 24

Table 3.2: Anthropometric Data for Subjects.............................. 24

Table 4.1: Productivity Results (Jumper)............................... 50

Table 4.2: Arm Elevation Results (Jumper).............................. 50

Table 4.3: Wrist Flexion/Extension Results (Jumper)........................ 51

Table 4.4: Wrist Radial/Ulnar Deviation Results (Jumper).................... 51

Table 4.5: Productivity Results (Spring) ................................ 59

Table 4.6: Arm Elevation Results (Spring).............................. 59

Table 4.7: Wrist Flexion/Extension Results (Spring)........................ 60

Table 4.8: Wrist Radial/Ulnar Deviation Results (Spring).................... 60

Table 5.1: Breakdown of alternative couplings during unconstrained conditions (data

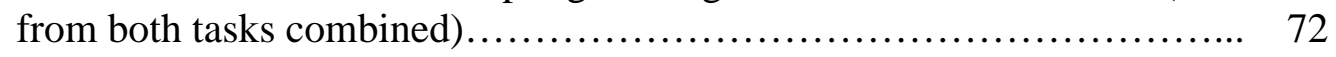

Table 5.2: Number of trials for which a different coupling was used during the unconstrained condition on the two orientations (data from both tasks

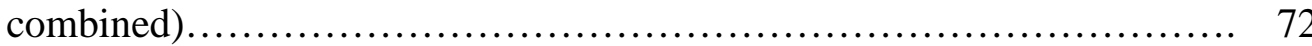

Table 7.1: Pilot Subject Anthropometry Data.............................. 83

Table 7.2: Pilot Subject Preferred Work Heights and Averages for Jumper Task..... 83

Table 7.3: Pilot Subject Preferred Work Heights and Averages for Spring Task....... 83

Table 7.4: Differences Between Task/Orientations Average Height $\left(\mathrm{H}_{\mathrm{avg}}\right)$ and Three Body Landmarks for each pilot subject........................... 83

Table 7.5: Differences between subjects (body landmark $-\mathrm{H}_{\mathrm{avg}}$ ) and rank by least difference between subjects..................................... 84

Table 7.6: Example of major steps for a subject data-collection period............. 85 


\section{LIST OF FIGURES}

Figure 1.1: Sagittal view of straight-handle pliers and bent-handle pliers........... 10

Figure 3.1: Initial starting arrangement for Jumpers Task....................... 17

Figure 3.2: Spring Task with first four springs connected as required.............. 18

Figure 3.3: Wrist goniometers........................................ 19

Figure 3.4: Subject instrumented with wrist goniometers and magnetic sensors....... 19

Figure 3.5: Straight-handle pliers and bent-handle pliers...................... 20

Figure 3.6: Vertical and $45^{\circ}$ slanted work surface orientations used in experiment.... 21

Figure 3.7: Neutral-wrist data file collection posture........................... 26

Figure 4.1: Plot of Residuals for Variable = Productivity $($ Jumper $) \ldots \ldots \ldots \ldots \ldots \ldots \ldots . \ldots \ldots$

Figure 4.2: Normal Probability Plot for Variable = Productivity (Jumper)........... 33

Figure 4.3: Plot of Residuals for Variable = Arm Elevation (Jumper)............. 34

Figure 4.4: Normal Probability Plot for Variable = Arm Elevation (Jumper)......... 34

Figure 4.5: Plot of Residuals for Variable $=$ Wrist Flexion/Extension $($ Jumper $) \ldots \ldots \ldots .35$

Figure 4.6: Normal Probability Plot for Variable $=$ Wrist Flexion/Extension (Jumper) ................................................ 35

Figure 4.7: Plot of Residuals for Variable = Wrist Radial/Ulnar Deviation (Jumper).. 36

Figure 4.8: Normal Probability Plot for Variable = Wrist Radial/Ulnar Deviation

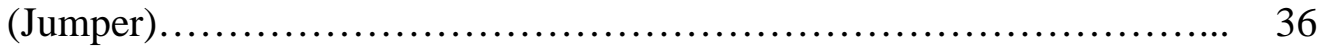

Figure 4.9: Plot of Residuals for Variable $=$ Productivity $($ Spring $) \ldots \ldots \ldots \ldots \ldots \ldots \ldots . \quad 37$

Figure 4.10: Normal Probability Plot for Variable = Productivity $($ Spring).......... 37

Figure 4.11: Plot of Residuals for Variable = Arm Elevation (Spring)............. 38

Figure 4.12: Normal Probability Plot for Variable = Arm Elevation (Spring)........ 38

Figure 4.13: Plot of Residuals for Variable $=$ Wrist Flexion $/$ Extension $($ Spring $) \ldots \ldots . . \quad 39$

Figure 4.14: Normal Probability Plot for Variable $=$ Wrist Flexion/Extension

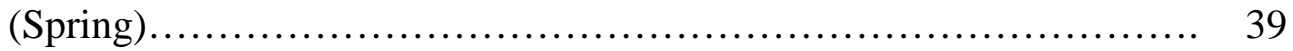

Figure 4.15: Plot of Residuals for Variable $=$ Wrist Radial/Ulnar Deviation (Spring). 40

Figure 4.16: Normal Probability Plot for Variable = Wrist Radial/Ulnar Deviation

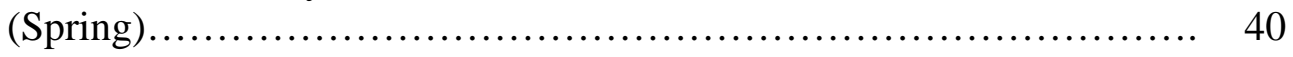

Figure 4.17: Time Order Plot for Productivity Residuals (Jumper)............... 41

Figure 4.18: Time Order Plot for Arm Elevation (Jumper)..................... 42

Figure 4.19: Time Order Plot for Flexion/Extension Residuals (Jumper)............ 43 
Figure 4.20: Time Order Plot for Radial/Ulnar Deviation Residuals (Jumper)........ 44

Figure 4.21: Time Order Plot for Productivity Residuals (Spring)................ 45

Figure 4.22: Time Order Plot for Arm Elevation (Spring).................... 46

Figure 4.23: Time Order Plot for Flexion/Extension Residuals (Spring)............ 47

Figure 4.24: Time Order Plot for Radial/Ulnar Deviation Residuals (Spring)......... 48

Figure 4.25: Interaction Effect of Pliers x Orientation on Arm Elevation (Jumper)... 52

Figure 4.26: Interaction Effect of Pliers x Constraint on Arm Elevation (Jumper)..... 52

Figure 4.27: Interaction Effect of Orientation x Constraint on Arm Elevation (Jumper)................................................... 53

Figure 4.28: Interaction Effect of Pliers x Orientation on Wrist Extension (Jumper)... 53

Figure 4.29: Interaction Effect of Pliers x Constraint on Wrist Extension (Jumper)... 54

Figure 4.30: Interaction Effect of Orientation x Constraint on Wrist Extension (Jumper) ................................................ 54

Figure 4.31: Interaction Effect of Pliers x Orientation on Ulnar Deviation (Jumper)... 55

Figure 4.32: Interaction Effect of Pliers x Constraint on Ulnar Deviation (Jumper)... 55

Figure 4.33: Interaction Effect of Orientation x Constraint on Ulnar Deviation

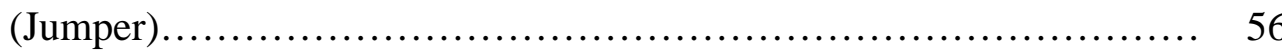

Figure 4.34: Main Effect of Pliers on Productivity (Jumper).................... 57

Figure 4.35: Main Effect of Orientation on Productivity (Jumper) ................ 57

Figure 4.36: Main Effect of Constraint on Productivity (Jumper)................. 58

Figure 4.36: Main Effect of Constraint on Wrist Extension (Jumper).............. 58

Figure 4.38: Interaction Effect of Pliers x Orientation on Productivity (Spring)....... 61

Figure 4.39: Interaction Effect of Orientation x Constraint on Ulnar Deviation

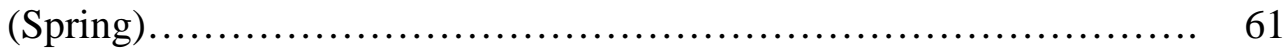

Figure 4.40: Main Effect of Pliers on Productivity (Spring) $\ldots \ldots \ldots \ldots \ldots \ldots \ldots \ldots \ldots . \quad 62$

Figure 4.41: Main Effect of Orientation on Productivity (Spring)................ 62

Figure 4.42: Main Effect of Constraint on Productivity (Spring)................. 63

Figure 4.43: Main Effect of Orientation on Arm Elevation (Spring).............. 63

Figure 4.44: Main Effect of Orientation on Wrist Extension (Spring).............. 64

Figure 4.45: Main Effect of Pliers on Radial/Ulnar Deviation (Spring)............. 64 


\subsection{INTRODUCTION}

\subsection{Cost to Business}

Any business interested in controlling costs needs to consider those related to employee injury and illness. Statistical data from the United States Bureau of Labor Statistics (BLS), show that for the year 2000 there were 1,664,018 injuries that lead to lost work time in this country (BLS, 2000). Of these, 577,814 (34.7\%) were categorized as musculoskeletal injuries (BLS, 2000).

According to the National Institute for Occupational Safety and Health (NIOSH), musculoskeletal disorders (MSDs) refer to conditions that involve the nerves, tendons, muscles, and supporting structures of the body (NIOSH, 1997). A 1999 report by the United States Department of Labor's, Occupational Safety and Health Administration (OSHA) stated that in 1995 MSDs cost employers an estimated $\$ 15$ to $\$ 20$ billion in workers' compensation costs and $\$ 45$ to $\$ 60$ billion more in indirect costs (OSHA, 1999). Furthermore, while the median number of days missed for all lost-time injuries was six in 2000, the number missed due to MSDs was seven (BLS, 2000). These statistics clearly indicate that the impact of MSDs on industry is large, and that there is a great need for research into the underlying causes of these disorders, and the development of effective interventions to reduce the numbers.

\subsection{Causes of Musculoskeletal Disorders}

The scope of this problem has lead to intense interest in understanding the causes of MSDs and their associated solutions. The development of a work-related MSD represents a complex interaction between three elements: an individual's psychological characteristics, the individual's physiological characteristics, and the mechanical/physical 
factors of the job or task (National Research Council and Institute of Medicine, 2001). The mechanical/physical factors represent all of the job or task characteristics that affect the physical loads experienced by the various body tissues. These may be obvious external loads, such as the weight of an object lifted or carried, or they may be less obvious internal stressors, such as a repetitive frictional force between a moving tendon and an underlying bone. The physiological characteristics represent an individual's unique biology, and how that relates to the imposed physical stressor. For example, factors such as age, gender, medical history, congenital conditions, tissue-healing rates, etc. can all influence an individual's physical state and how they respond to the physical demands of the job. Lastly, the psychological element represents an individual's psychological response to extrinsic stressors; this element is determined by such psychological characteristics as attitudes, values, and coping mechanisms

A common classification scheme for physical risk factors categorizes them as those due to excessive force, those caused by repetition, and those resulting from awkward postures (other risk factors in this classification scheme include vibration, temperature extremes, and contact stress) (Pulat, 1997; Bridger, 1995). Excessive force simply implies that the imposed load immediately exceeds tissue tolerance. For example most people would be unable to lift a box weighing $200 \mathrm{~kg}$. The role of repetition as a risk factor is explained in a model described by Marras (1999). In this model injury prevention is achieved by keeping tissue loads lower than the particular tissue tolerance (when load exceeds tolerance injury occurs). Repetition in this model is an exposure effect, whereby the imposed load may initially be lower than the tissue tolerance, but repetitive exposure of the same tissue with insufficient rest leads to lowering of the tissue 
tolerance to the point where the imposed load is greater. Lastly, awkward posture as a risk factor can be explained in a couple of different ways as outlined in the next section.

\subsubsection{Posture as a Physical Risk Factor}

All of these risk factors have been subject to empirical research (in isolation and in numerous combinations) concerning their contribution to work-related MSDs. The 1997 NIOSH publication extensively reviewed the epidemiological evidence for the causal relation between different physical risk factors and MSDs of the back, neck and upper extremities (NIOSH, 1997). This review concluded that there is epidemiological evidence for an association between posture and some MSDs, including those of the low back, the neck and shoulder, and tendonitis of the hand/wrist; in many cases, the strength of association is higher when awkward posture is combined with other risks factors.

Additionally, though there is insufficient evidence of posture alone being causally related to carpal tunnel syndrome, if combined with other risk factors (particularly repetition) there is evidence for this association as well. (NIOSH, 1997)

There are a number of proposed mechanisms by which non-neutral postures may cause MSDs. For one, most muscles are at an optimal force-production length when the joint they span is in a neutral range (usually close to the mid-range of motion) (Konz, Johnson, 2000). Deviations away from the neutral position can negatively affect the musculoskeletal geometry, placing the muscles at non-optimal lengths, where there is a higher metabolic cost for a given force requirement (Chaffin, Andersson, and Martin, 1999; Wells, 1999). As a result, fatigue may occur more quickly, and if the duration of work is long or recovery time short, an overexertion injury may result. 
With more massive body segments, non-neutral work postures can have the simple effect of increasing the load due to gravity (Chaffin et al, 1999). At the higher work load the muscle must work harder, leading to quicker fatigue and potential for injury, as described in the previous paragraph. Additionally, at a higher work load an increase in intramuscular pressure can impair blood flow, leading to ischemic conditions and injury of nearby tissue (Viikari-Juntura, 1999). The shoulder is one region that is particularly susceptible to this effect, with arm elevation as little as $30^{\circ}$ (with no hand load) demonstrating higher intramuscular pressures and reduced blood flow (Herberts, Kadefors, Hogfors, Sigholm, 1984).

Another potential source of injury is soft tissue impingement in non-neutral postures. Under these circumstances soft tissues may be compressed against bone or other hard tissues (Chaffin et al, 1999; Marklin, 1999). In the case of blood vessels this impingement can jeopardize blood flow, while with tendons it can result in increased internal force on the tendon (Chaffin et al, 1999). The latter case is the foundation for a mechanism proposed by Armstrong and Chaffin (1979) for explaining the contribution of posture to carpal tunnel syndrome, whereby the flexor retinaculum and carpal bones act as pulleys for the tendons of the forearm flexors in flexion and extension. As pulleys these structures exert a reaction force on the tendons as they curve around the structure, and the magnitude of the force is directly proportional to the angle of deviation of the joint.

Another proposed relationship between posture and injury, specific to carpal tunnel syndrome, is the idea that deviations from neutral increase carpal tunnel pressure. Previous research has demonstrated that, in both CTS patients and healthy subjects, there 
is an increased pressure within the carpal tunnel with flexion/extension and radial/ulnar deviations of the wrist (Noah, Weiss, Gordon, Bloom, Yuen So, Rempel, 1995; Keir, Bach, Engstrom, Rempel, 1996; Rempel, Bach, Gordon, Yuen So, 1998). Currently there is not an adequately supported model explaining the rise in pressure with deviation, however, it has been demonstrated that with carpal tunnel pressures as little as $30 \mathrm{~mm} \mathrm{Hg}$, subjects experience neurophysiological changes and symptoms of paresthesia (Lundborg, Gelberman, Minteer-Convery, Lee, 1982); suggesting this as a potential mode of injury.

\subsection{Implications for Hand-Tool Design}

\subsection{1 "Bend the Tool"}

While it is important to remember that MSDs are a result of complex interaction among the elements described above, nevertheless, it is often convenient to provide general guidelines for the design of work. A review of a number of textbooks that specifically address hand/wrist posture and the design of hand-tools leads to the ubiquitous recommendation to "bend the tool, not the wrist" (Kroemer, Kroemer, Kroemer-Elbert, 1994; Chaffin et al, 1999; Bridger, 1995; Pulat 1997). The rationale behind this recommendation is that the conventional in-line design of tool handles can require users to adopt awkward postures under certain conditions of use, and that it is preferable to alter the tool design to maintain more neutral wrist (and often shoulder) posture.

\subsubsection{Previous Research on Bent-Handle Tools}

\subsubsection{Hammers}

There have been a number of empirical studies that have explored the above tenet. Granada and Konz (1981) and Krohn and Konz (1982) performed subjective evaluation 
studies of bent-handle hammers versus a conventional straight-handle hammer design. In the 1981 study Granada and Konz found that users preferred two versions of a benthandle design (one design had a shorter handle) compared to the straight-handle hammer, which served as the reference. In the 1982 study the authors constructed a number of hammers with varying degrees of bend in the handle $\left(10^{\circ}, 15^{\circ}, 21^{\circ}, 26^{\circ}\right.$, and $\left.32^{\circ}\right)$.

Subjects pounded in two nails using each of the five different bent-handle hammers and a straight handle hammer. The hammers were ranked for preference and the top three were used to hammer one additional nail, after which the final three were re-ranked. In this study it was found that the hammer with the $10^{\circ}$ bend was preferred, followed by the straight-handle hammer, and then the $15^{\circ}$ bend.

In a 1984 study by Konz and Street the three highest ranked hammers (from the 1982 study) were evaluated using performance measures and subjective evaluation again. In the study the subjects were required to use each of the three different hammers to set a nail (using a "tap"), followed by a single "pound" strike. Each hammer was used on two practice nails and then two trials of five nails; these final ten nails provided the data for analysis of performance measure. It was found that for the dependent variables, depth of penetration and nail deviation from vertical, there were no significant differences between the three hammers. On the subjective evaluation the hammers were ranked in the same order as the earlier study. In the discussion section these authors concluded that, at least in a short experimental run, subjects prefer a bent handle design and that for the variables they measured, there are no performance decrements. They do state additionally that hammer handle design is more than just the angle of bend, and that other 
factors such as work orientation, hand position, grip circumference, and balance point need to be considered.

Similarly, Schoenmarklin and Marras (1989a, 1989b) evaluated the effect of bending the handle of a hammer on a number of outcomes, including dynamic wrist posture and range-of-motion (ROM), muscle fatigue, performance measures (driving force, accuracy, and number of misses), and subjective evaluation. Their study had hammer handle design as an independent variable, with three types of hammer (straight, $20^{\circ}$ bend, and $40^{\circ}$ bend), as well as work orientation with two levels (vertical and horizontal surfaces). Their results indicated that although there were significant differences between the three hammers in mean ulnar deviation at time of impact (with the straight-handle having the greatest ulnar deviation, and the $40^{\circ}$ bent-handle having the least), the total ROM in the radial /ulnar plane was not significantly different between the different hammer designs. There was only one significant postural effect of work orientation, that being on wrist flexion.

For the performance measures there were significant results for all variables due to work orientation, but there were no significant effects of hammer design. In the companion article (1989b) the results indicated that hammer handle design had no significant effect on either muscle fatigue or subjective rating of body discomfort (work orientation did provide significant results for both of these measures). These authors conclude that, "applying the established ergonomic principle of bending the tool and not the wrist to the hammer could produce less biomechanical stress on the wrist" - this due to the fact that there was approximately equal deviation in both the radial and ulnar directions with the bent-handle designs, while the straight-handle hammer had greater 
ulnar deviation - "while maintaining the performance of a straight hammer" (p. 410).

But they also point out that, "changes in tool design should not be considered independently of the task" (p.411).

\subsubsection{Files}

Application of a bent handle design to files by Hsu and Chen (1999) also showed a number of favorable results. Their study consisted of two tasks using five different files: one conventional straight file and four files constructed with varying degrees of bend between the filing surface and the handle $\left(50^{\circ}, 60^{\circ}, 70^{\circ}\right.$, and $\left.90^{\circ}\right)$. For the first task subjects took 1,000 strokes on a standardized iron cube; the dependent variables in this case were radial/ulnar deviation of the right wrist, task efficiency (measured as the mass of material filed from the cube, fatigue (measured as the reduction in grip force after task performance), and subjective preference. For the other task subjects were given 20 minutes to make even an iron plate; the dependent variable was measured as the surface area of the plate that extended greater than $16 \mu \mathrm{m}$ from the plate surface (subjective preference was again evaluated for this task). In both tasks all subjects used all five files, and a randomized complete block design was used with blocking on subject.

For the postural outcome significant effects were found by file type. The straighthandle file had the highest mean $\underline{\text { ulnar deviation }}\left(23.2^{\circ}\right)$, followed by the $50^{\circ}$ bent-handle $\left(6.9^{\circ}\right)$, and then the $60^{\circ}\left(3.9^{\circ}\right)$ and $70^{\circ}\left(4.0^{\circ}\right)$ handle bend designs (the last two were not significantly different from each other). Finally the $90^{\circ}$ bent-handle file had a mean radial deviation of $9.2^{\circ}$. For task efficiency the $90^{\circ}$ bent-handle file was significantly less productive than the other four files, while all others were not significantly different from one another. The measure of fatigue found no significant difference between the four 
bent-handle designs, while use of the straight-handle file resulted in significantly reduced grip strength when compared to all bent-handle designs. The test of precision did not find any significant differences between any of the five designs. Finally, in both tasks there were significant differences in the subjective preference, with the $60^{\circ}$ bent-handle design being most preferred in both cases, followed by the $50^{\circ}$ and $70^{\circ}$ designs. The authors summarize their findings to conclude that the $60^{\circ}$ bent-handle file demonstrates superior results compared to the straight-handle file, but they also identify that the modified handle design is inappropriate for working in constrained or limited spaces. They suggest that workers should have both a conventional file (for specifically constrained work) and a bent-handle file in their repertoire to reduce the risk of workrelated MSDs.

\subsubsection{Knives}

In an evaluation of poultry cutting Fogleman, Freivalds, and Goldberg (1993) analyzed video data of workers engaged in poultry cutting to create two bent-handle knives for evaluation; the two designs incorporated a bend of $+30^{\circ}$ and $-30^{\circ}$ (respectively) measured from the axis of the handle. Subjects used these two knife designs, as well as a conventional straight-handle knife to perform two different types of cut: 1) a hanging cut (vertical work surface), and 2) table cut (horizontal work surface). Note: when using the $-30^{\circ}$ bend-handle knife subjects used a dagger grip (blade exiting from the ulnar aspect of the hand).

The investigators recorded a number of measures of posture (as well as other dependent variables of lesser interest here). The results (limited to those most related to this investigation) revealed that for the hanging cut condition the knife with the $+30^{\circ}$ 
angle minimized the flexion/extension ROM, ulnar/radial ROM, maximum wrist flexion, and maximum wrist extension. For the table cut the $-30^{\circ}$ angle design (with dagger grip) minimized flexion/extension ROM, maximum extension, and maximum ulnar deviation. They concluded that for the two types of cuts tested, the straight knife was the worst in regards to posture, that the $-30^{\circ}$ bent-handle design (dagger grip) was best for the table cut, and that the $+30^{\circ}$ bent-handle design was best for the hanging cut.

\subsubsection{Pliers}

One of the most familiar (at least from the literature), and oft-cited examples of the "bend the tool" design principle is the bent-handle pliers (see Figure 1.1) and the work of Tichauer $(1968,1973,1975,1976,1977)$. In these numerous publications Tichauer provides a web of cross-references to his previous works; all of which appear to be based on two different experiments.

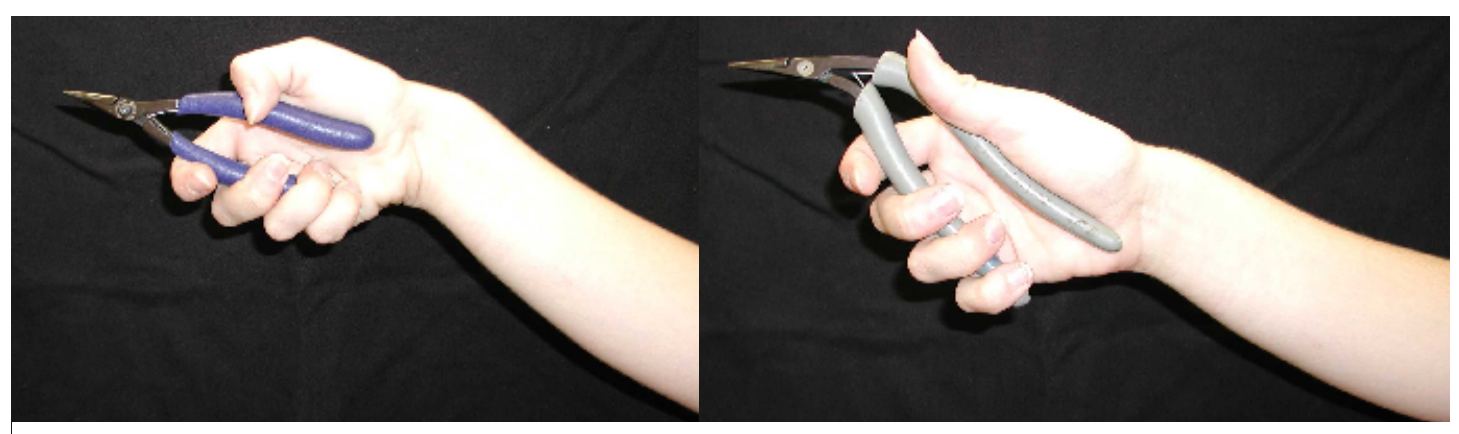

Figure 1.1: Sagittal view of straight-handle pliers (left) and bent-handle pliers (right). 
The Tichauer (1968) manuscript does not provide a detailed description of the experimental task, nor are any statistical results reported. The experimental task is inferred from the document to be as follows: Two groups of subjects performed a forearm rotation task under two different conditions. In one case the subjects gripped a handle connected to a "kinematometer" with a T-handle, which kept the wrist straight, while in the other condition the subjects used "a straight screwdriver handle, which causes the wrist to be deviated towards the ulna" (Tichauer, 1968; p 156).

Experimental data for only one subject are provided in Tichauer (these are not reproduced or discussed here due to lack of utility, but the interested reader is referred to Figure 7, p 158 in Tichauer, 1968); showing results for angular displacement, velocity, acceleration, and biceps myogram under the two conditions. The author goes on to report the results as follows:

"In the course of an experiment conducted with more than forty volunteers it was found that when the angle of ulnar deviation of the wrist exceeded thirty degrees the biomechanical parameters of performance efficiency: displacement, velocity, and acceleration of the shaft decreased suddenly while myoelectric activity was doubled. Thus, at the risk of some oversimplification, it may be stated that the subject had to labor twice as hard to produce half the output". (p 157)

Further, the results of subjective physical response are provided in a figure that shows four out of 40 subject complaints under the "wrist straight" condition compared with 25 out of 40 complaints under the " $40^{\circ}$ ulnar deviation" condition (Tichauer, 1968, p 159).

In his 1973 work Tichauer discusses the results of a field study. In this study two groups of trainees in an electronics assembly job take part in a 12-week training program for a wire-twisting task. One group of 40 trainees uses the conventional straight-handle 
pliers, while another group of 40 uses the bent-handle pliers. At the end of the 12 -week period the author reports that of those participants using straight pliers, 25 out of 40 suffered from injuries classified as either tenosynovitis, epicondylitis, or carpal tunnel syndrome, compared with only four (out of 40) employees who used pistol-grip pliers for the same task (1973).

An x-ray image (similar to figure 1.1) that Tichauer used frequently (and sometimes the Tichauer results as well) often accompanies the "bend the tool, not the wrist" design recommendation in the ergonomics textbooks. Furthermore, Tichauer's results are frequently referenced within the other studies on hand-tool design.

Considering the seemingly impressive results reported by Tichauer one would expect additional research supporting the bent-handle pliers, along with evidence of use of the tool in industry. This investigator was unable to find any examples from industry for which the tool is currently being used, and a review of the ergonomics literature indicated that the only other empirical research done on bent-handled pliers was that of Dempsey and Leamon (1995). (Lewis and Narayan (1993) did a review of handle design, but it lacked empirical research.)

The Dempsey and Leamon (1995) investigation sought to further examine the theoretical advantages that the bent-handle pliers seem to provide. They conducted a laboratory investigation designed to mimic the original wire-twisting task that Tichauer reported on in 1973. In this experiment 14 subjects used both straight-handle and benthandle pliers to rotate a modified screw (milled to allow subjects to grasp the head with the jaws of the pliers) mounted on a vertical work surface. These investigators wished to investigate the effect of height as well, and so the task was performed at five different 
heights (elbow height $(\mathrm{EH}), \mathrm{EH}+/-5$ ", and $\mathrm{EH}+/-10$ "). At each combination of pliers by height, subjects performed five repetitions of a one-minute task during which they were instructed to rotate a screw as many times as possible. The dependent variables in this study were productivity, measured as revolutions per minute, and subjective preference.

There was a main effect of pliers in this study, with the bent-handle pliers resulting in a mean of 2.25 rotations per minute (8.25\%) less than the straight-handle pliers. These investigators also found a significant interaction between type of pliers and work height; these results showed that although there were performance decrements at all heights with the bent-handle pliers, the largest decrement in performance occurred at elbow height.

For the subjective response (subjects were asked which pliers they would choose to use if the task performed was their full-time job) nine subjects stated a preference for the straight-handle pliers, while five chose the bent-handle pliers. These authors also noted "several subjects reported that the bent pliers were more comfortable but were slower in use, and that they would (therefore) select the straight pliers. Performance was never mentioned by the experimenter". 


\subsection{BENT-HANDLE TOOLS IN INDUSTRY}

\subsection{Proposed Rationale for Lack of Acceptance}

A visual inspection of the two images in Figure 1.1 intuitively suggests that this design would allow work to be performed in more neutral postures. However, there are potential problems with this assumption. First, the image suggests that the tool is to be used with the jaws aligned with the long axis of the forearm; this may not be the case, and this assumption cannot be made without a thorough understanding of the particular task that the pliers will be used for. If, for example, the task is going to require the worker to manipulate a piece or part in a number of different orientations and/or along different axes then the bent-handle design may lose its postural advantage. Similarly, the specificity of the handle design may constrain the user's coupling with the tool to a power grip or an oblique grip. (With the power grip all four fingers are wrapped around the handle and are opposed by the thumb, and often the handle is perpendicular to the forearm axis. An oblique grip is a modified power grip where the thumb is aligned with the axis of the tool - Konz \& Johnson, 2000). Additionally, the lack of symmetry along the long-axis of the tool may reduce the worker's options for manipulating the pliers, leading to productivity decrements.

If the constraints imposed by the tool design are not immediately outweighed by a productivity advantage, then potential users may be inclined to not use the bent-handle tool. This would seem particularly true considering the insidious nature musculoskeletal injuries. 


\subsection{Hypotheses}

This research seeks to explore the reason why the bent-handle (often called pistol-grip Konz \& Johnson, 2000) pliers are apparently unpopular in industry despite the conventional wisdom supporting their superior design. This will be examined by testing the following hypotheses:

1. It is hypothesized that there will be a significant productivity disadvantage with the bent-handle pliers due to the constraint of the tool design (lack of symmetry about the long axis).

2. It is hypothesized that when the tool is held with a power grip or oblique grip, postural benefits (less radial/ulnar deviation and less arm elevation) of the benthandle pliers are task-specific. In particular, it is expected that a task that can be performed with the pliers remaining in the sagittal plane and not requiring rotation out of this plane will have significantly less deviations with the bent-handle pliers. Conversely, tasks requiring manipulation out of this plane will favour the straighthandle pliers.

3. It is hypothesized that the specificity of the design of the bent-handle pliers will result in significant postural benefits (less radial/ulnar deviation and less arm elevation) over the straight-handle pliers when the pliers are held (constrained) to a power grip or oblique grip. Conversely, when subjects are able to hold the pliers unconstrained the postural advantage will be lost. 


\subsection{MATERIALS AND METHODS}

\subsection{Experimental Tasks}

The experiment consisted of two separate tasks that were designed to represent expected real-world applications for which bent-handle pliers may be recommended for neutral posture promotion. Both tasks (described in detail below) involved parts manipulation in a relatively small work area directly in front of the subject. One of the tasks (Jumpers) required small sagittal and lateral movements of the pliers, with essentially no tool rotation, while the other task (Springs) required similar sagittal and lateral movements, as well as rotational manipulation of the tool.

\subsubsection{Jumper Task}

The first task required subjects to move five (5) red computer jumpers laterally between two sets of computer connectors. The connectors were standard 0.1" motherboard mounting pins organized in two columns of pins, separated by 0.1 " $(0.254 \mathrm{~cm})$, both between pin columns and pin rows. For this experiment two 36-pin (18 x 2) connectors were used. The two connectors were mounted vertically parallel on a board, side by side, separated by $5.6 \mathrm{~cm}$ (see Figure 3.1 ). On both sets of connectors a black jumper was placed on the first (top) pair of pins and (counting down) on the $13^{\text {th }}$ pair of pins, these four jumpers were not moved and served as boundary demarcations. Five red jumpers were placed on the intervening pairs of pins such that there was always an empty pair of pins above and below each red jumper; this was done to prevent interference between jumpers. The jumpers themselves were insulated gold contacts enclosed in a plastic housing, described as standard 0.1" $\mathrm{x} 0.3$ " jumpers. 


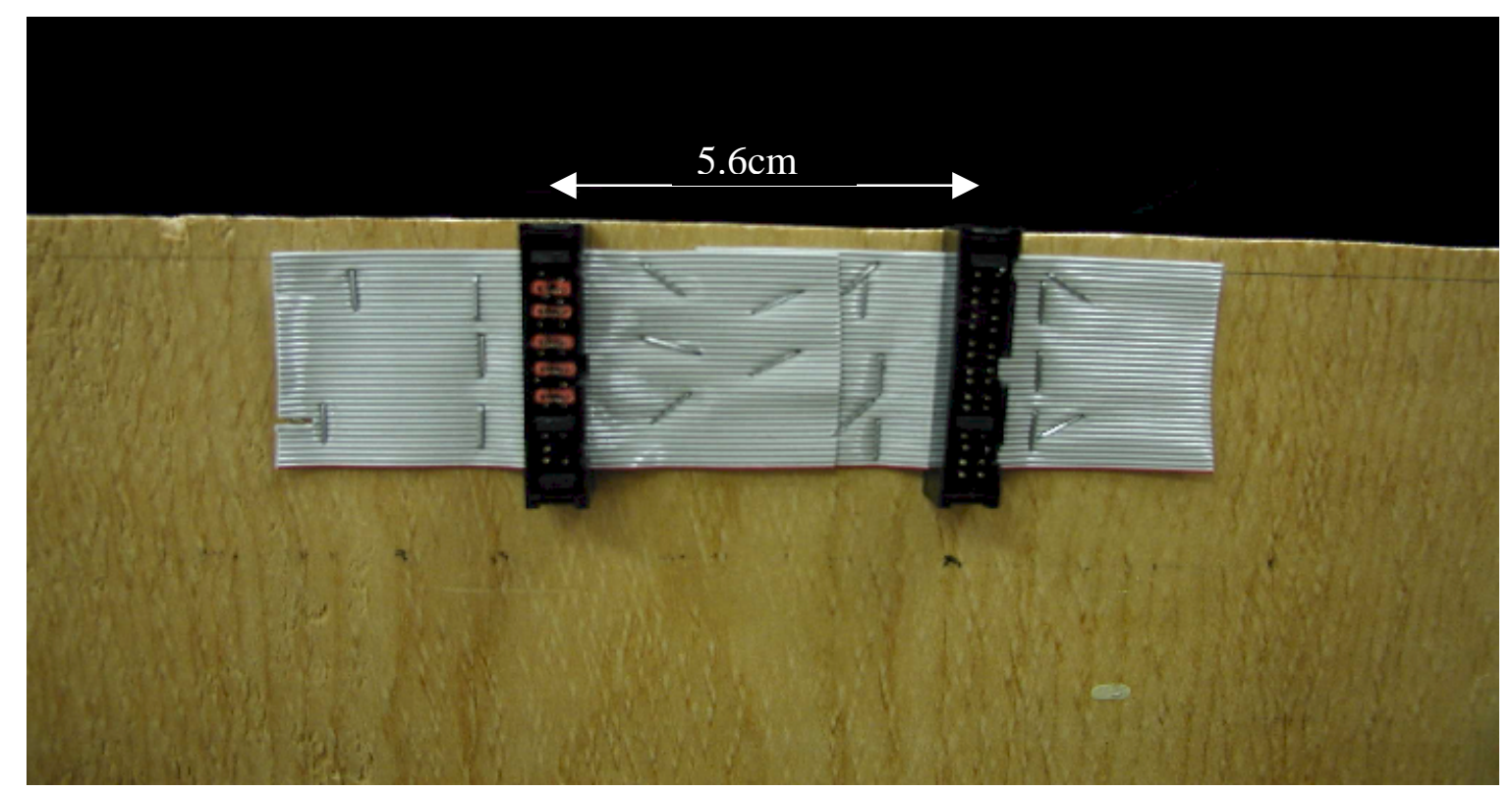

Figure 3.1: Initial starting arrangement for Jumpers Task.

The initial starting condition had the five red jumpers in position on the left connector. A cycle consisted of grasping the jumpers, one at a time, and moving them all from their initial pin positions on the left connector to the same pin positions on the right connector, followed by moving them all back to their initial starting position on the left connector. When moving the jumpers from one connector to the other, the subjects always started by moving the top jumper first and worked down.

\subsubsection{Spring Task}

The other task required subjects to sequentially connect to a post, the free end of springs mounted at the other end to a board. Ten stainless steel extension springs $(0.312$ " x $0.0286 "$ x $2.50 "$; spring rate $=0.55 \mathrm{lb} / \mathrm{in}$; max. deflection $=4.27$ ”) were fastened at one end to a board, as shown in Figure 3.2. A finishing nail $(1.8 \mathrm{~mm}$ shaft diameter; $2.8 \mathrm{~mm}$ head diameter) was placed $7.7 \mathrm{~cm}$ to the right of the mounting point; the nail was mounted leaving $12.5 \mathrm{~mm}$ exposed to serve as the post. Subjects were instructed to use the pliers to grasp the free-end loop of the spring and (pulling against the spring tension) attach it to 
the post. Subjects started at the top left spring, worked down the column of springs, and after attaching the bottom left spring, moved to the top right spring and again worked down the column. Once all the springs were attached, the subjects reversed their work by individually removing the springs from the posts; when unfastening the springs, the subjects started at the bottom left spring, worked up, and then unfastened the right column starting at the bottom. The subjects were required to grasp the loop with the pliers to detach it from the post. Attaching all ten springs, followed by unfastening all ten springs constituted a complete cycle. Note: because of the two different orientations of the work surface (vertical and slanted, described below under Independent Variables), the springs always hung down when unattached. However, the orientation of the loop was variable, requiring subjects to variably manipulate the pliers in rotation to grab the loop.

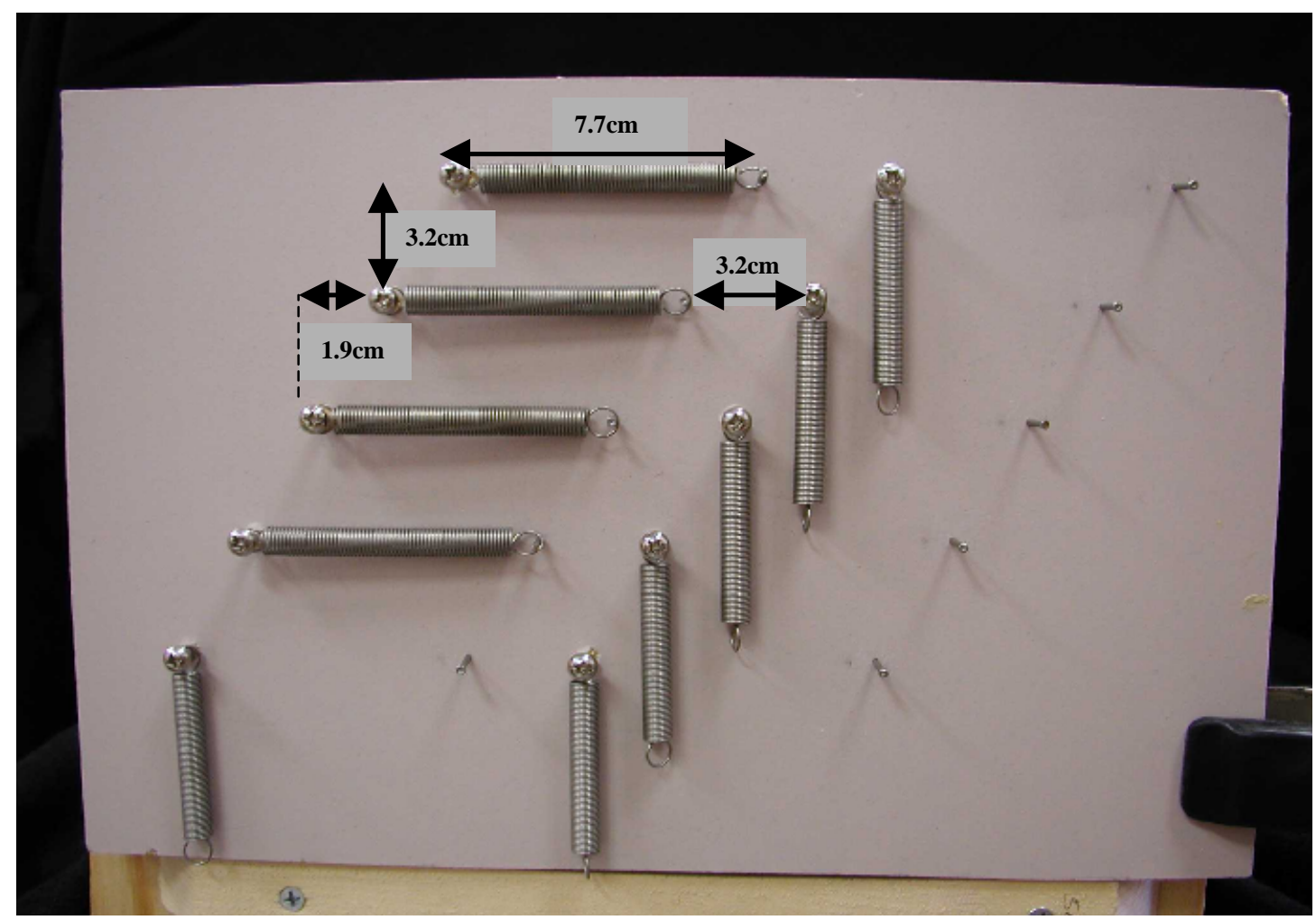

Figure 3.2: Springs Task with first four springs connected as required. 


\subsection{Apparatus}

Time to complete trials (productivity) was measured using a Sportline ${ }^{\circledR}$ Alpha 410 hand-held stopwatch, measured to the hundredths of a second. The wrist flexion/extension angle and angle of radial/ulnar deviation was measured with two wrist goniometers; these are a non-commercial product designed to independently collect angle data based on the angular displacement measured by a rotary potentiometer. This apparatus is described in Marras and Schoenmarklin (1993); and is shown in Figures 3.3 and 3.4. The three-dimensional shoulder posture data (measured as arm elevation from the vertical) were captured by the Ascension Flock of Birds system (Ascension Technology ${ }^{\circledR}$, VT, U.S.A.) magnetic tracking system, and were recorded with Innovative Sports Training Motion Monitor ${ }^{\odot}$ software (version 4.10). (Figure 3.4 displays the Flock of Birds magnetic sensors on a subject.)

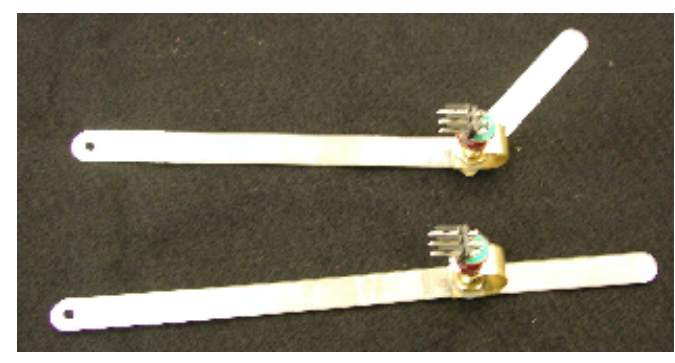

Figure 3.3: Wrist goniometers.

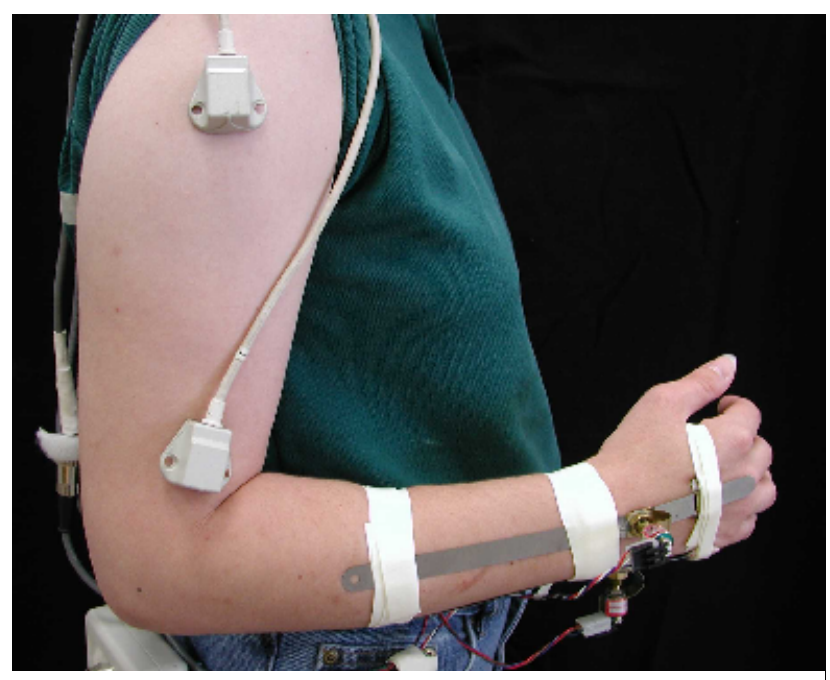

Figure 3.4: Subject instrumented with wrist goniometers and magnetic sensors. 


\subsection{Independent Variables}

\subsubsection{Pliers}

Two levels of pliers were applied, straight-handle (conventional) and benthandle (pistol grip) (see Figure 3.5). The straight-handle pliers were Swanstrom model S325E. The bent-handle pliers were Swanstrom model S325EPR. Both pliers had the same jaw characteristics and dimensions: serrated jaw; jaw length $=27 \mathrm{~mm}$; tip width $=$ $1.50 \mathrm{~mm}$; tip thickness $=1.50 \mathrm{~mm}$. The overall length of the straight pliers was $15.8 \mathrm{~cm}$; the overall length of the bent pliers was $19.2 \mathrm{~cm}$.

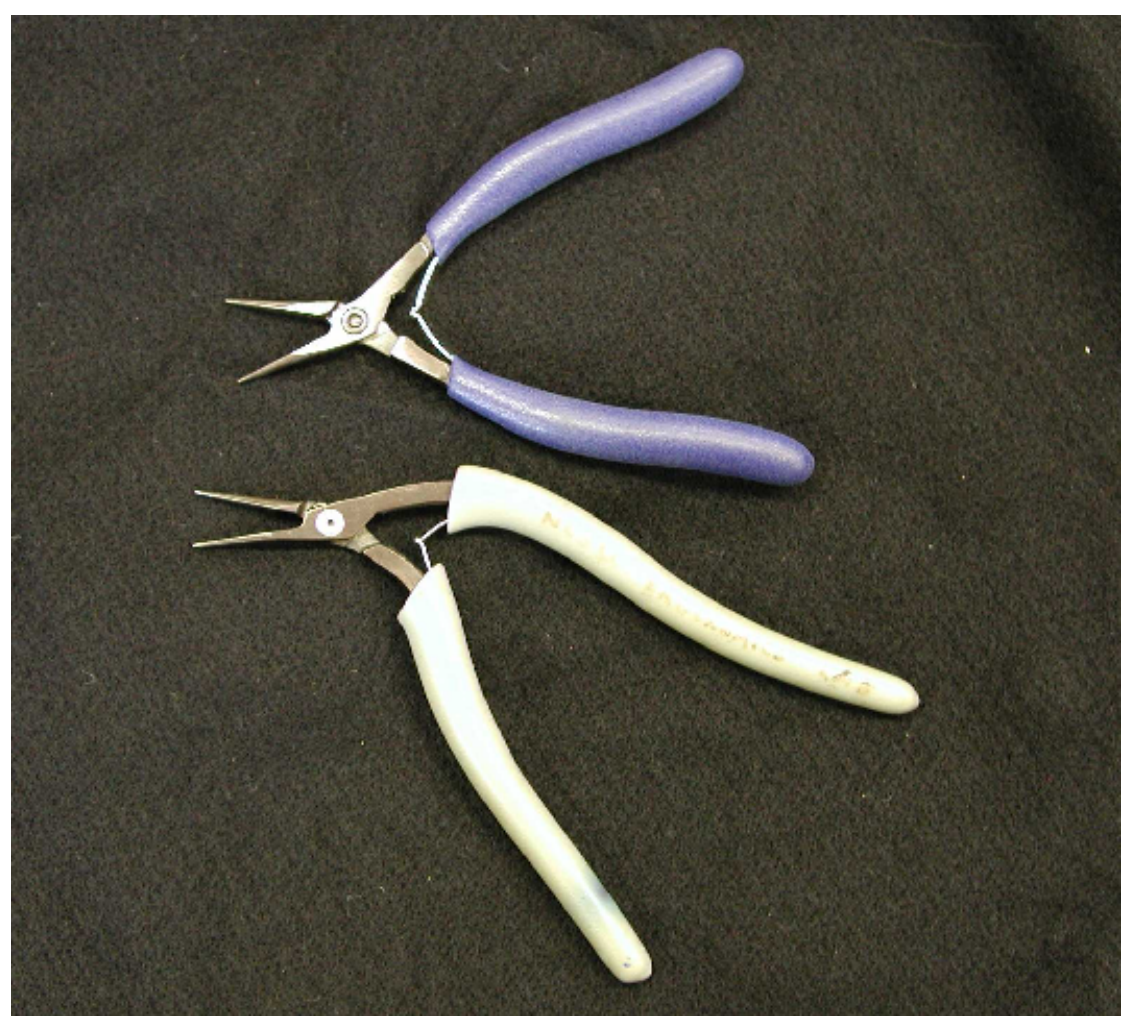

Figure 3.5: Straight-handle pliers (top) and bent-handle pliers (bottom) used in experiment. 


\subsubsection{Constraint}

Subjects used the pliers under two different constraint conditions. In the constrained condition subjects were required to hold the pliers within the palm of the hand using either a power grip or an oblique grip (described earlier in Section 2.1). The constrained grip also required subjects to hold the pliers with the jaws (or active end) extending from the radial aspect of the hand. In the unconstrained condition subjects were allowed to hold the pliers in any manner desired (including the manner described for the constrained condition).

\subsubsection{Work Surface Orientation}

Two different work surface orientations were used (Figure 3.6), vertical and slanted at a $45^{\circ}$ angle.

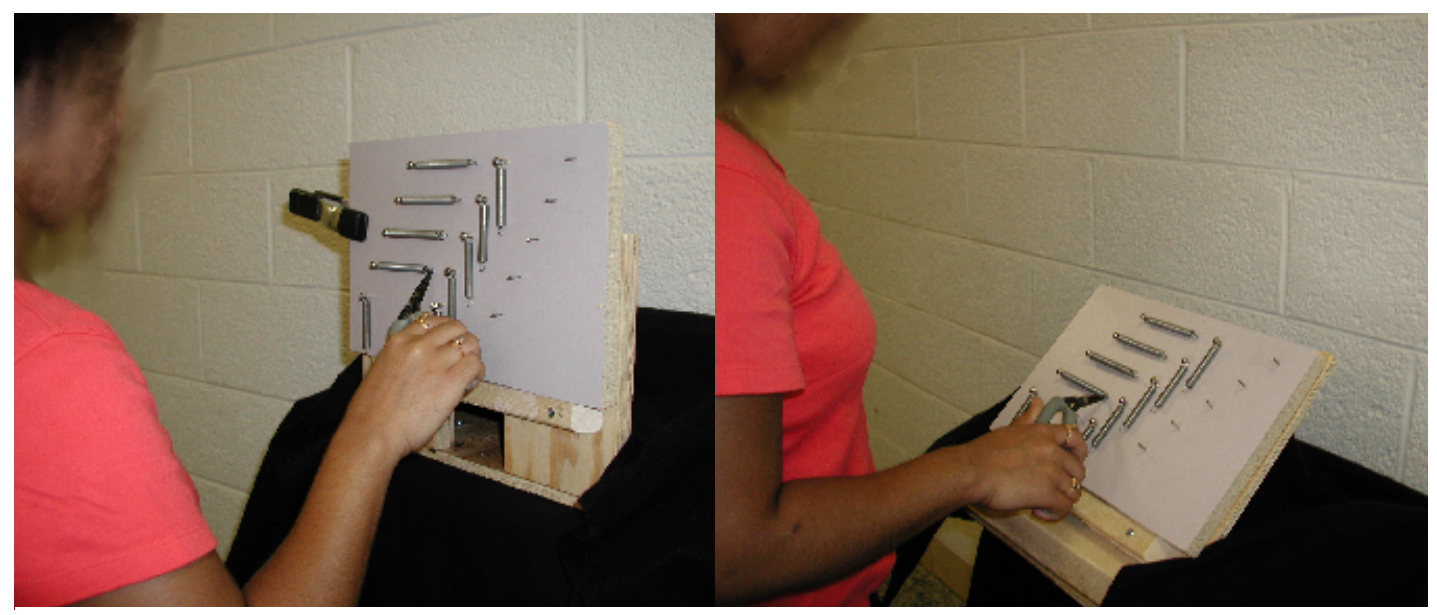

Figure 3.6: Vertical (left) and $45^{\circ}$ slanted (right) work surface orientations used in experiment. 


\subsection{Dependent Variables}

\subsubsection{Productivity}

Productivity was measured as the time (measured to the hundredth of a second) taken to complete three cycles of a given condition.

\subsubsection{Posture}

Postural data were collected for the right wrist and right shoulder.

\subsubsection{Wrist}

Mean flexion/extension and radial/ulnar deviation postural data were determined for the third cycle of task completion for all conditions. Data in the flexion/extension, and radial/ulnar planes were collected at $300 \mathrm{~Hz}$ using the wrist goniometers described above (refer to Figure 3.5 and 3.6). These data were entered via an A/D board into Data Translation Inc. ${ }^{\circledR}$ Global Lab ${ }^{\oplus}$ (version 3.00), which was loaded on a Compaq ${ }^{\circledR}$ notebook computer. Exported data provided data files in ASCII format at the same rate of $300 \mathrm{~Hz}$, with 0-4096 bits covering the range of angular displacement of the potentiometers.

\subsubsection{Shoulder}

Mean shoulder posture data were determined for the third cycle of task completion for all conditions. Shoulder posture was measured as elevation of the upper arm from the vertical; in this way the calculated values represented a combination of shoulder flexion and abduction. Three-dimensional positional data for two sensors secured to the right upper arm (see Figure 3.4) were collected at $60 \mathrm{~Hz}$ using the MotionMonitor@ system; the raw data were low-pass filtered by a Butterworth filter with an effective cut-off frequency of $20 \mathrm{~Hz}$. The three-dimensional data were processed to produce arm elevation values at the same rate of $60 \mathrm{~Hz}$. 


\subsection{Working Height}

An important consideration in this experiment was the working height for the different experimental conditions, since working height has a direct effect on the dependent variables (particularly posture). A large source of variability would be introduced if subjects had been allowed to determine their own preferred working height (some preferring higher heights to promote visual acuity, while some preferring lower heights to promote neutral body postures); to overcome this, pilot work was done to determine a standardized working height for each of the four conditions of task (jumpers and springs) and work surface orientation (vertical and $45^{\circ}$ slanted).

Three subjects with ergonomics backgrounds (two PhD's and one Master's student) performed the two tasks at the two different orientations, using both pairs of pliers (a total of eight conditions). In all cases, the pilot subjects were instructed to hold the pliers in the constrained manner. For each of the eight conditions, these pilot subjects were asked to perform the task at any, and as many heights of their choosing in order to determine their preferred static working height for each of the eight different conditions. For a given combination of task and orientation the average of the two preferred heights (by type of pliers) was calculated. This value $\left(\mathrm{h}_{\mathrm{avg}}\right)$ was evaluated against three landmarks on the respective pilot subject: the acromion process, the lateral epicondyle of the elbow, and the mid-distance between these two landmarks (mid-arm).

For the four conditions (two tasks $\mathrm{x}$ two orientation), the difference between $\mathrm{h}_{\text {avg }}$ and the mid-arm landmark resulted in minimization of the differences among the three subjects. Finally, the average for the three subjects of $\left(\mathrm{h}_{\mathrm{avg}}\right.$ - mid-arm) was determined for the four different combinations of task and orientation; these average distances from 
the mid-arm (rounded to the nearest centimeter) were set as the working heights at which all subjects would perform the various tasks. Data from the pilot work is shown in the appendix in Tables 6.1-6.5, while the work heights determined from this work are shown in Table 3.1. The heights shown in Table 3.1 were used for both the constrained and unconstrained conditions of the four different task by orientation combinations

Table 3.1 Work Surface Heights for both Tasks by Orientation.

\begin{tabular}{|l|c|}
\hline Task by Orientation Combination & $\begin{array}{l}\text { Work Surface Height } \\
\text { Relative to Mid-Arm Height }\end{array}$ \\
\hline Jumper x Slanted Work Surface & $-17 \mathrm{~cm}$ \\
\hline Jumper x Vertical Work Surface & $0 \mathrm{~cm}$ \\
\hline Spring x Slanted Work Surface & $-10 \mathrm{~cm}$ \\
\hline Spring x Vertical Work Surface & $0 \mathrm{~cm}$ \\
\hline
\end{tabular}

\subsection{Subjects}

Sixteen subjects (eight male, eight female) were recruited from the general university population. Subjects ranged in age from 22 to 38 years, with mean age equal to 28.25. All subjects were right-handed and had normal, or corrected-to-normal vision. Subject stature ranged from $155.6 \mathrm{~cm}$ to $196.1 \mathrm{~cm}$, with a mean of $173.4 \mathrm{~cm}$. The height to the acromion process of the right shoulder and to the lateral epicondyle of the right elbow was measured in the standing upright position; the mean values, ranges and standard deviations are shown in Table 3.2.

Table 3.2: Anthropometric Data for Subjects

\begin{tabular}{|c|c|c|c|c|}
\hline & Mean & SD & Minimum & Maximum \\
\hline Age & 28.25 & 4.753946 & 22 & 38 \\
\hline Stature & 173.3688 & 10.93586 & 155.6 & 196.1 \\
\hline Acromion & 142.8438 & 10.219 & 127.6 & 164.8 \\
\hline Lateral epicondyle & 110.475 & 7.344522 & 99.8 & 127.2 \\
\hline Mid-Arm Height & 126.6594 & 8.75426 & 113.7 & 146 \\
\hline
\end{tabular}




\subsection{Protocol}

\subsubsection{Anthropometry}

Subjects were given a brief description of the purpose of the study and the protocol to be followed, after which the subject signed an informed consent form. Anthropometric data (stature, height to right acromion process and right lateral epicondyle of the humerus) were collected; the heights from the ground to the acromion process and from the ground to the lateral epicondyle of each subject were averaged to determine the subject's mid-arm height (see Appendix, Table 6.6 for mean, standard deviation, and ranges); this height was used as the reference point for determining each subject's working heights as described previously.

\subsubsection{Instrumentation}

The subject had two wrist goniometers (measuring flexion/extension and radial/ulnar deviation) applied to the right arm as described by Marras and Schoenmarklin (1993). Two magnetic sensors were applied to the right arm with adhesive tape. One sensor was positioned on the lateral aspect of the upper arm, near the elbow, with the other being positioned on the lateral aspect of the upper arm nearer the shoulder (see Figure 3.4). The sensors were positioned so as to not interfere with arm motion.

\subsubsection{Neutral File Collection}

After application of instrumentation the subject was seated with her/his right elbow flexed to $90^{\circ}$ and her/his shoulder abducted $90^{\circ}$ to horizontal (see figure 3.7). The arm was positioned on a supporting horizontal surface with the lateral epicondyle, the wrist center of rotation (in the coronal plane), and the third metacarpal inline. 
Additionally, the arm was positioned so that the lateral epicondyle, the dorsum of the wrist and the third metacarpal-phalangeal joint were all in the same plane. Once in this position, a five second wrist goniometer file was collected. The subject then removed her/his arm from the support, dropped the arm to a vertical position, and returned it to the horizontal surface where it was again positioned in the manner just described. Another five-second data collection was performed. These two files served as "neutral" calibration files.

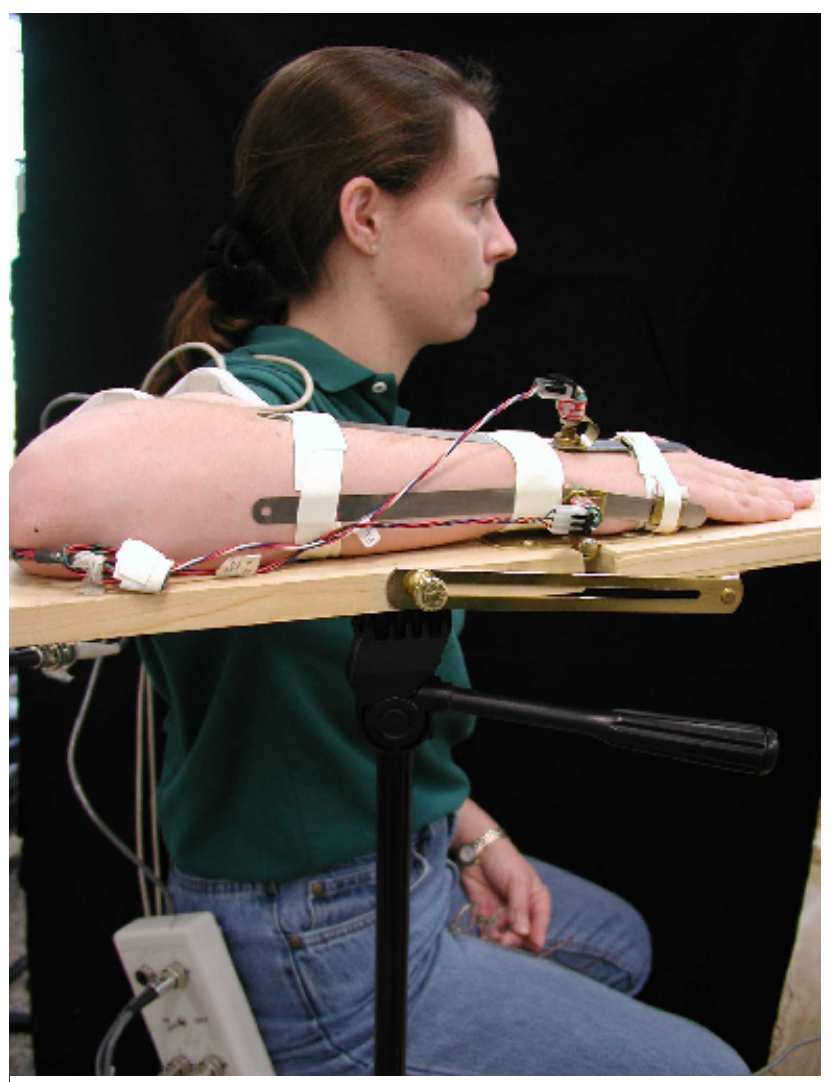

Figure 3.7: Neutral-wrist data file collection posture. 


\subsubsection{Trial Randomization}

All subjects performed all eight conditions (pliers by orientation by constraint) for the spring task followed by all conditions for the jumper task. Within each task, the order of performance of the four conditions determined by orientation and pliers was randomized. Within this randomization, all subjects first performed the task constrained followed by the unconstrained condition.

\subsubsection{Subject Instructions}

Prior to performance of the randomized trials, the particular task was explained to the subject. The subject was told that she/he was free to position herself/himself in a standing position in front of the work surface however she/he felt comfortable, and that she/he was allowed to hold the work surface with her/his left hand, but that she/he could not use the left hand to assist the task performance in any other manner. The investigator demonstrated task performance, and the subject was told that her/his objective was to perform the task as quickly as possible under all conditions.

\subsubsection{Subject Practice}

At this point all subjects performed a practice session during which they completed a minimum of two cycles of the task in the vertical orientation with both the straight pliers and the bent pliers (order of performance by pliers was alternated between subjects), followed by performance of one cycle of the task in the slanted condition with both types of pliers. Pilot data had indicated that asymptotic performance would occur within this period. All subjects were videotaped and a piece-by-piece timed analysis of the videotape was done to ensure that such was the case. Refer to the appendix (Table 6.7) for a summary of a sample subject data collection session. 


\subsubsection{Trial Data Collection}

During performance of the 16 conditions (two tasks $\mathrm{x}$ two pliers $\mathrm{x}$ two orientations $\mathrm{x}$ two constraints) data collection was performed in the following manner: Standing in front of the work surface, the subject was asked if she/he was ready, if so, she/he was instructed to begin completion of three cycles of the task. The subject was periodically reminded to perform as quickly as possible at the beginning of trials. The time to complete three cycles was measured for all trials. Also, at the start of the third cycle for all trials, wrist goniometer and arm elevation data were collected. At the end of the third cycle data collection was concluded. After each constrained condition, subjects were asked to take some time to explore other manners for holding the pliers to determine if there was an alternate way that they would prefer to hold the pliers. During this time the subject was able to try the task with any grip she/he wanted. Once the subject determined the grip that she/he preferred, the unconstrained trial was conducted. As noted previously, the unconstrained condition could be performed using the same grip as in the constrained condition, if that was the subject's preference. Any alternative grips used were noted in a lab notebook.

After all 16 trials were completed another five second, neutral wrist goniometer trial was collected. This concluded the subject's involvement in the experiment. 


\subsection{Data Analysis}

\subsubsection{Data Processing}

\subsubsection{Productivity}

For each subject the time to complete three cycles of each of the conditions was recorded as a measure of productivity; no data processing was required.

\subsubsection{Wrist Posture}

All subjects had 19 data files: three neutral-wrist files (two taken prior to trial completion, and one taken after), and one file for each of the 16 trials. Each file contained three channels of data: one for flexion/extension, one for radial/ulnar deviation, and one for a trigger (which was used to indicate the start and finish of the third cycle of trial completion). The two initial neutral-wrist files provided a reference value for the 16 trials, which were processed to provide a mean value for both flexion/extension and radial/ulnar deviation for the last cycle of each trial.

All files were converted to ASCII output using the built-in StatPack software. The ASCII output files were in bits (0-4096). A Basic program was created to compute the mean bit-value for both flexion/extension and radial/ulnar deviation for the two initial neutral-wrist files. The mean values for both variables (flexion/extension and radial/ulnar deviation) from the two files were then averaged to provide an overall average neutral value for each variable. These values in bits were then converted to voltages and ultimately angles (in degrees) using a linear calibration equation. Within the same program, the mean bit-value was calculated for both channels of the two variables for each of the 16 trials, which were then converted to angles (in degrees) using the same linear calibration equation. The average values from the neutral files (flexion/extension 
and radial/ulnar deviation) were subtracted from the appropriate values for each of the 16 trials, producing the mean wrist angle over the course of the third cycle of each trial, for both flexion/extension and radial/ulnar deviation.

(Note: the data from the neutral file taken at the end of each subject's data collection period was used to provide confirmation that the position of the wrist goniometers had not changed by more than $5^{\circ}$ during data collection; this was done by comparing the final neutral values for each goniometer to the neutral trials collected at the start of the data collection period.)

\subsubsection{Shoulder Posture}

Each subject had 16 activity files from the MotionMonitor $^{\circledR}$ system, which provided data to calculate the mean arm elevation. These files were converted to export files using built-in conversion software. The output from the exported files was 3dimensional positional data for the two points on the upper arm. A Basic program was created to use trigonometry to convert these data into arm elevation (from vertical) values at the same rate of $60 \mathrm{~Hz}$. The data for each trial was then averaged to provide one value for arm elevation over the course of the third cycle of each condition.

\subsubsection{Statistical Analysis}

A statistical model, based on the Randomized Complete Block described by Kolarik (1995) was developed for this investigation. An analysis of variance (ANOVA) was performed on this model to test the effects of the independent variables on the dependent variables and their interactions.

Tests of Assumptions: Model residuals were predicted and plotted and the Shapiro-Wilk's test was conducted to determine whether the residuals were normally 
distributed. Testing for equality of the variance of the response variables was achieved through a time-order plot of each of the response variables, blocked by subject. The assumption of an independent random sample was achieved through the subject recruitment process employed. Since all the independent variables tested had only two levels, there was no need to perform a post-hoc Tukey's test.

In all cases of the dependent variable (productivity, wrist flexion/extension, wrist radial/ulnar deviation, and arm elevation) the same model applied, with the response variable represented by $\mathbf{Y}$. In each case the overall mean is represented by $\boldsymbol{\mu}$. The a term represented the effect of the two types of pliers (straight and bent). The ? term represented the effect of the two work surface orientations (vertical and slanted). The d term represented the effect of the two levels of constraint (constrained and unconstrained). The $\boldsymbol{B}$ term represented the effect of subject blocking. Additionally, the model contained terms to represent the three two-way interactions between $\mathbf{a}, ?$, and $\mathbf{d}$, as well as the three-way interaction between these terms. Finally, the model included an error term, e. The model is as follows:

$$
Y_{i, j, k, l}=\mu+a_{i}+?_{j}+d_{k}+\beta_{1}+\left(a_{i} ?_{j}\right)+\left(a_{i} d_{k}\right)+\left(?_{j} d_{k}\right)+\left(a_{i} ?_{j} d_{k}\right)+e_{i, j, k, l}
$$

In all cases the F-statistic was tested as the ratio of the mean square of the treatment $\left(\mathrm{MS}_{\text {treatement }}\right)$ divided by the mean square of the error term $\left(\mathrm{MS}_{\text {error }}\right)$, where;

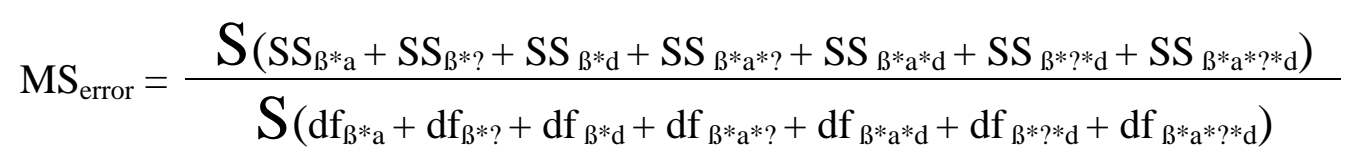




\subsection{RESULTS}

\subsection{Tests of Assumptions}

\subsubsection{Test for Assumption of Normal Distribution of Residuals}

\subsubsection{Jumper Task}

Figures 4.1 through 4.8 show the results of the test for normality for the dependent variables and the corresponding Shapiro-Wilk's probability for the jumper task. 


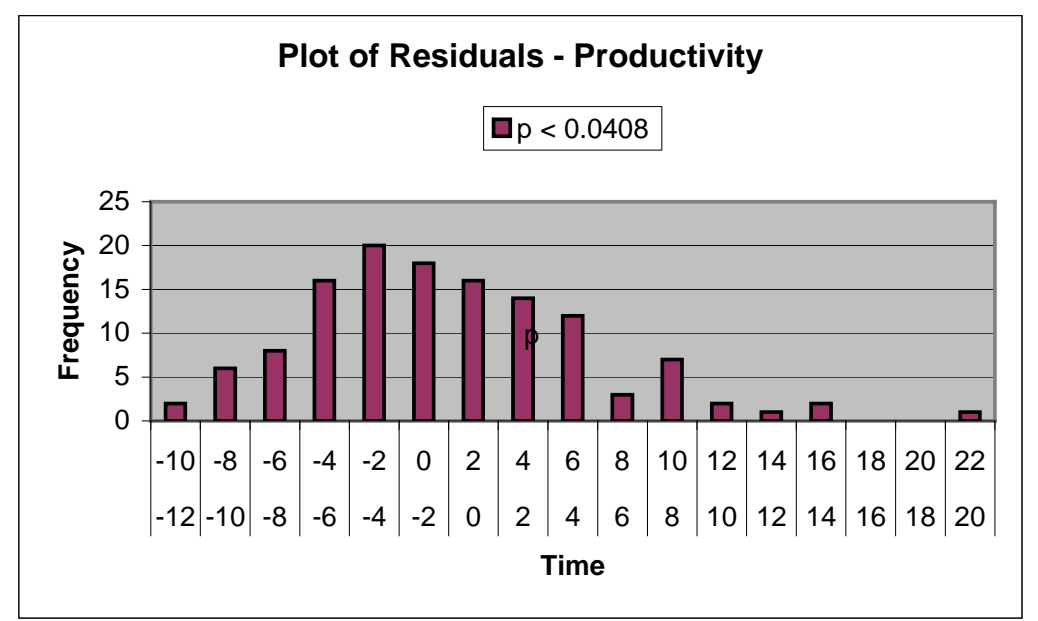

Figure 4.1: Plot of Residuals for Variable = Productivity .

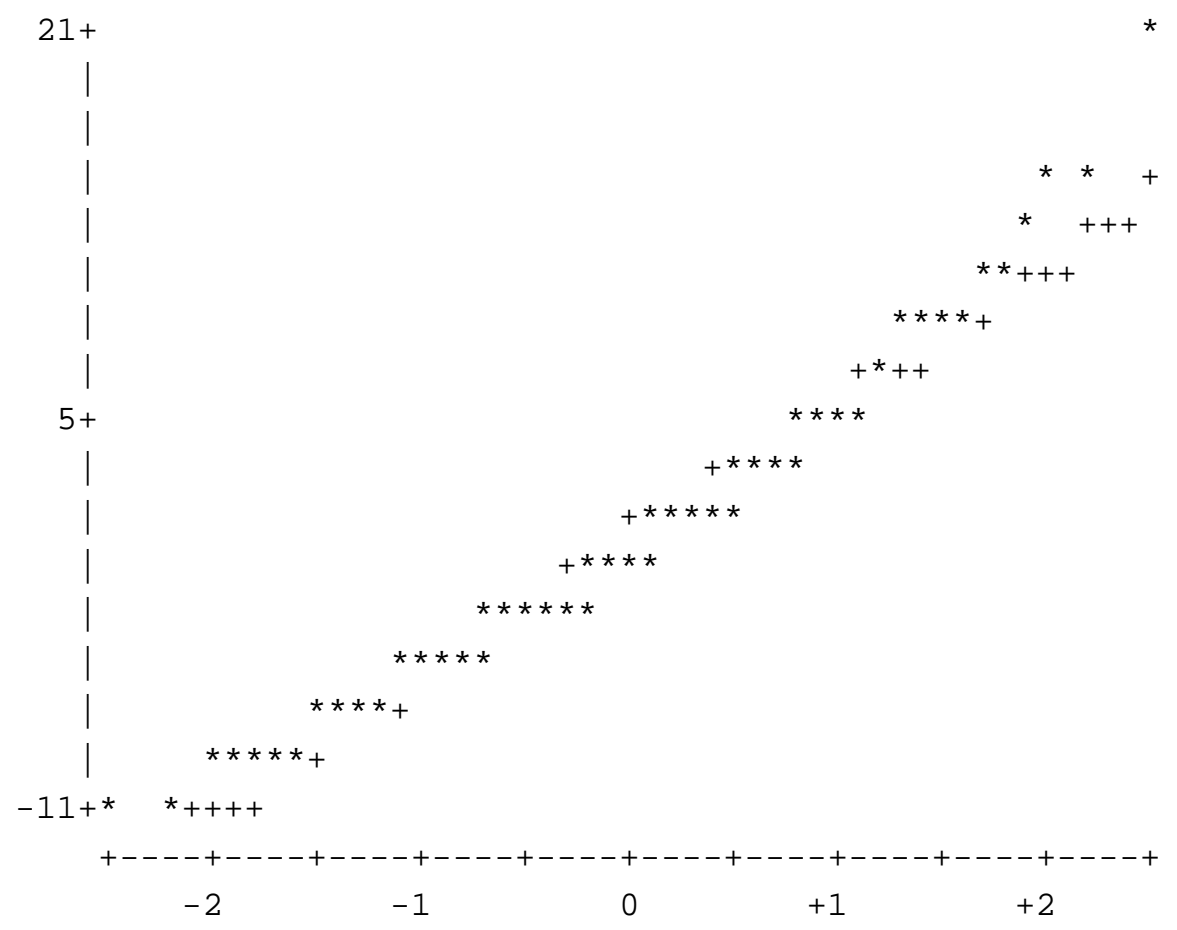

Figure 4.2: Normal Probability Plot for Variable $=$ Productivity 


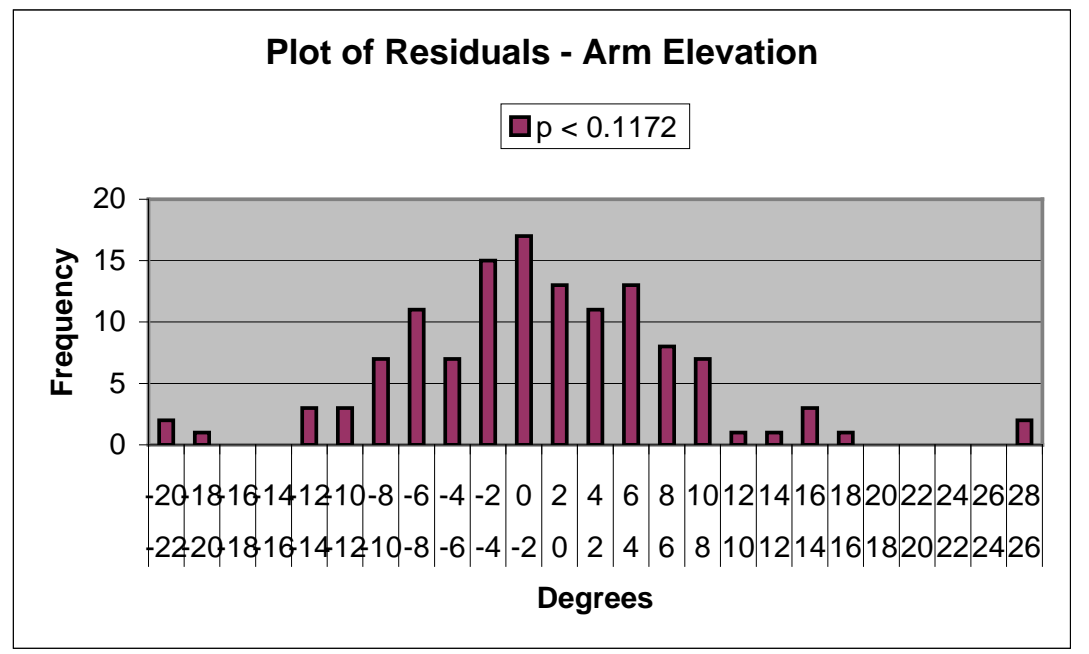

Figure 4.3: Plot of Residuals for Variable = Arm Elevation .

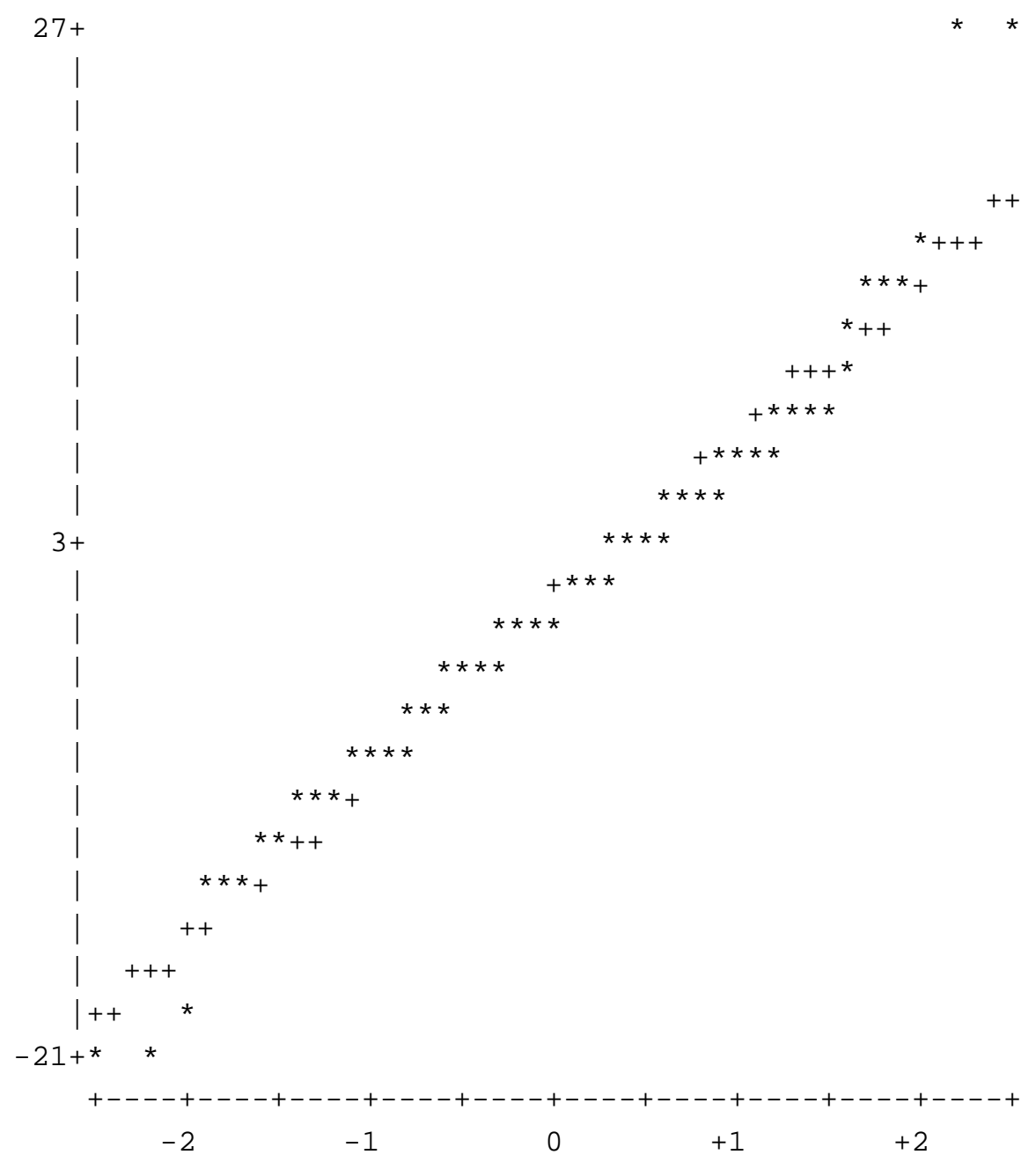

Figure 4.4: Normal Probability Plot for Variable = Arm Elevation. 


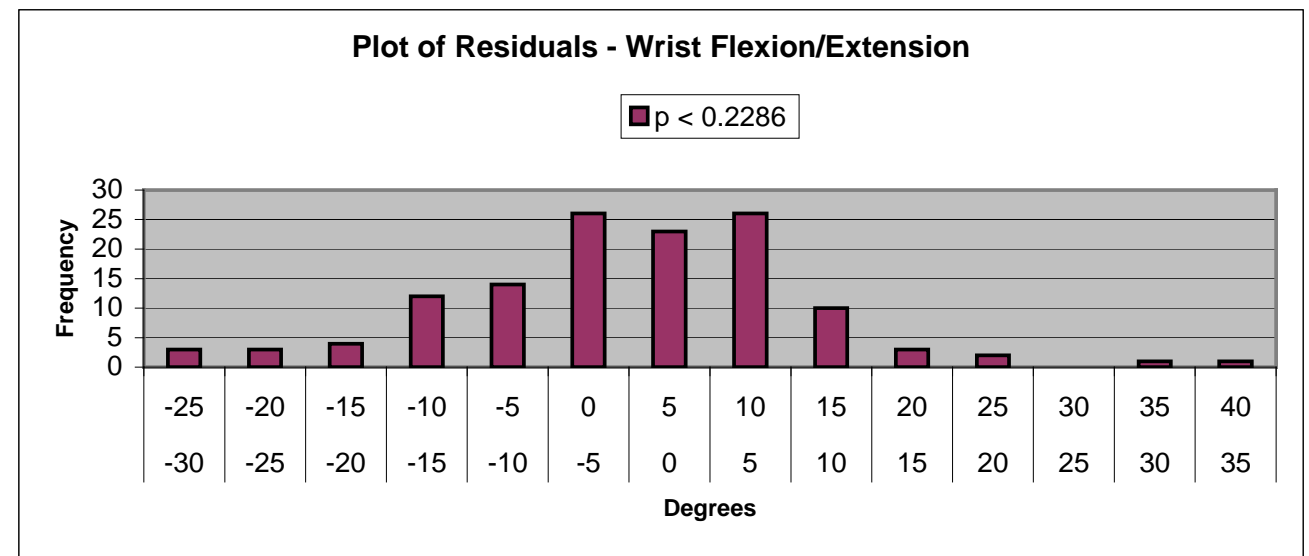

Figure 4.5: Plot of Residuals for Variable $=$ Wrist Flexion/Extension.

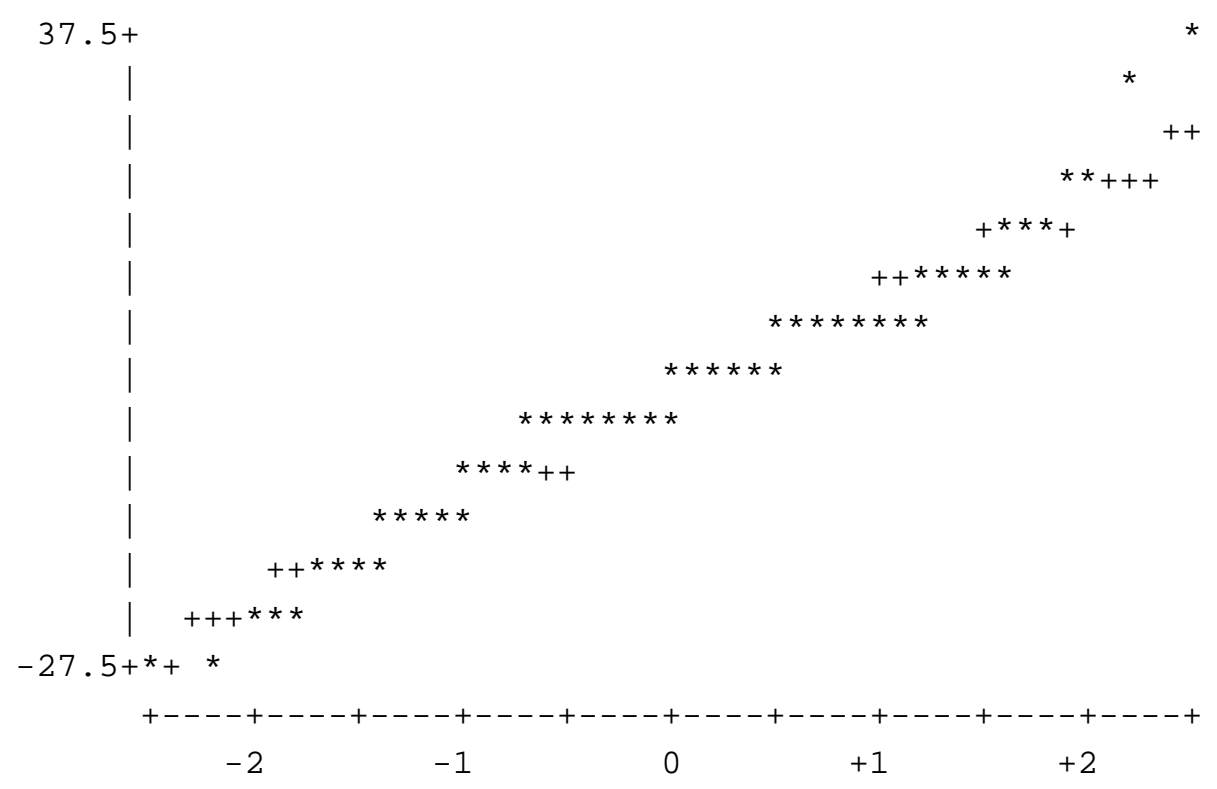

Figure 4.6: Normal Probability Plot for Variable $=$ Wrist Flexion/Extension. 


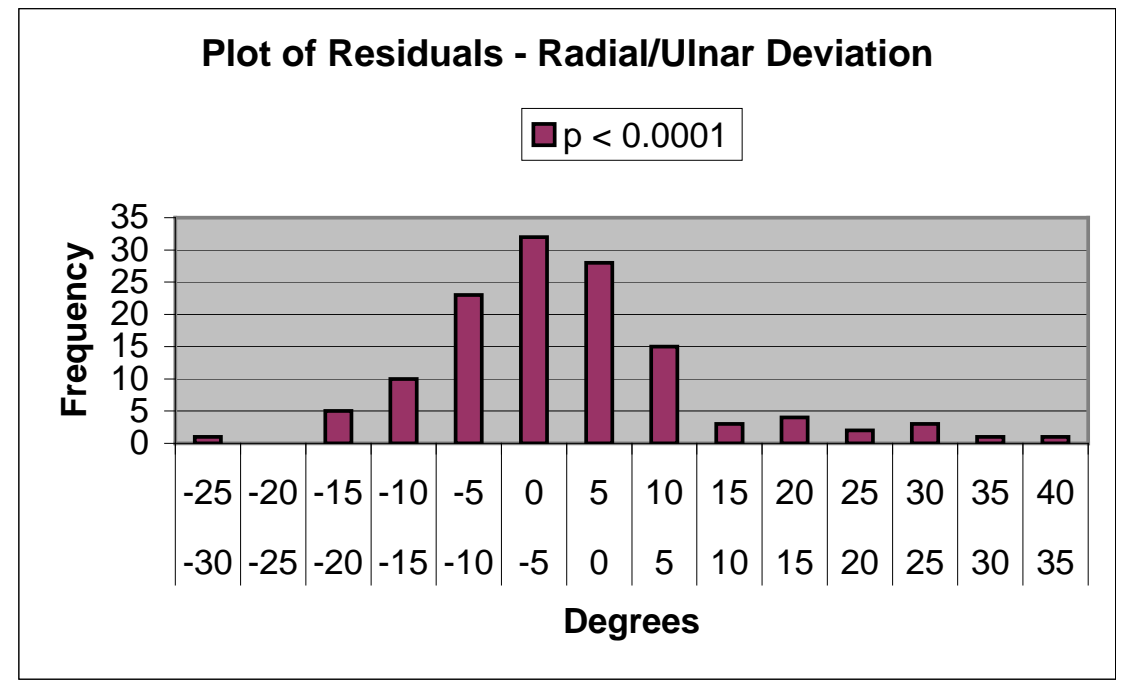

Figure 4.7: Plot of Residuals for Variable = Wrist Radial/Ulnar Deviation.

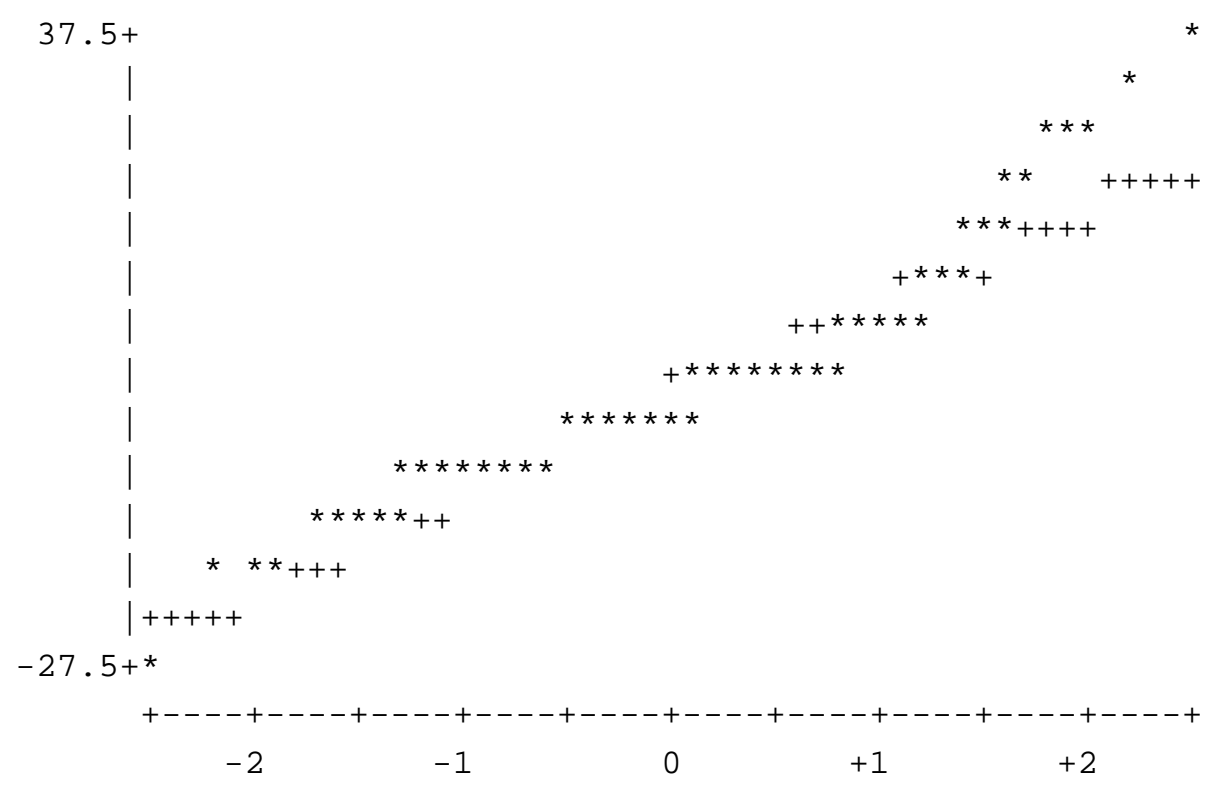

Figure 4.8: Normal Probability Plot for Variable $=$ Wrist Radial/Ulnar Deviation . 


\subsubsection{Spring Task}

Figures 4.9 through 4.16 show the results of the test for normality for the dependent variables and the corresponding Shapiro-Wilk's probability for the spring task. Note that the extreme outliers in Figure 4.15 are a result of three instances when subjects used a "dagger" grip when performing a task on the vertical work surface; this put the wrist in a position where it was deviated to a large degree in the radial direction.

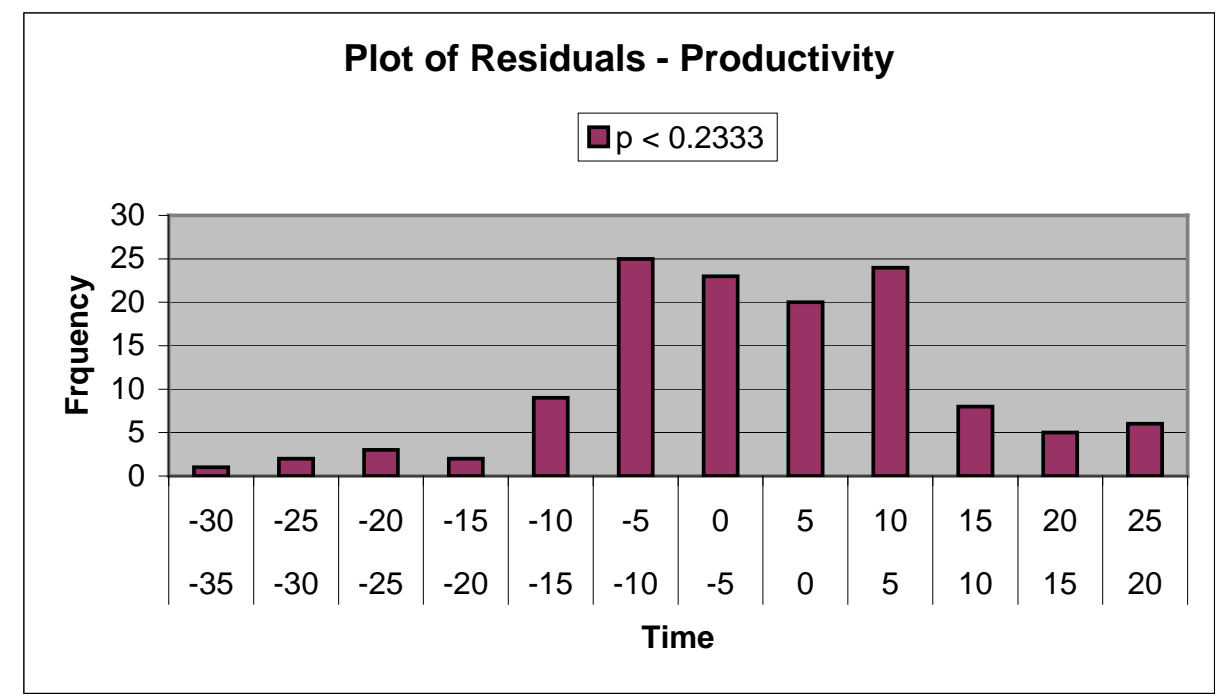

Figure 4.9: Plot of Residuals for Variable = Productivity .

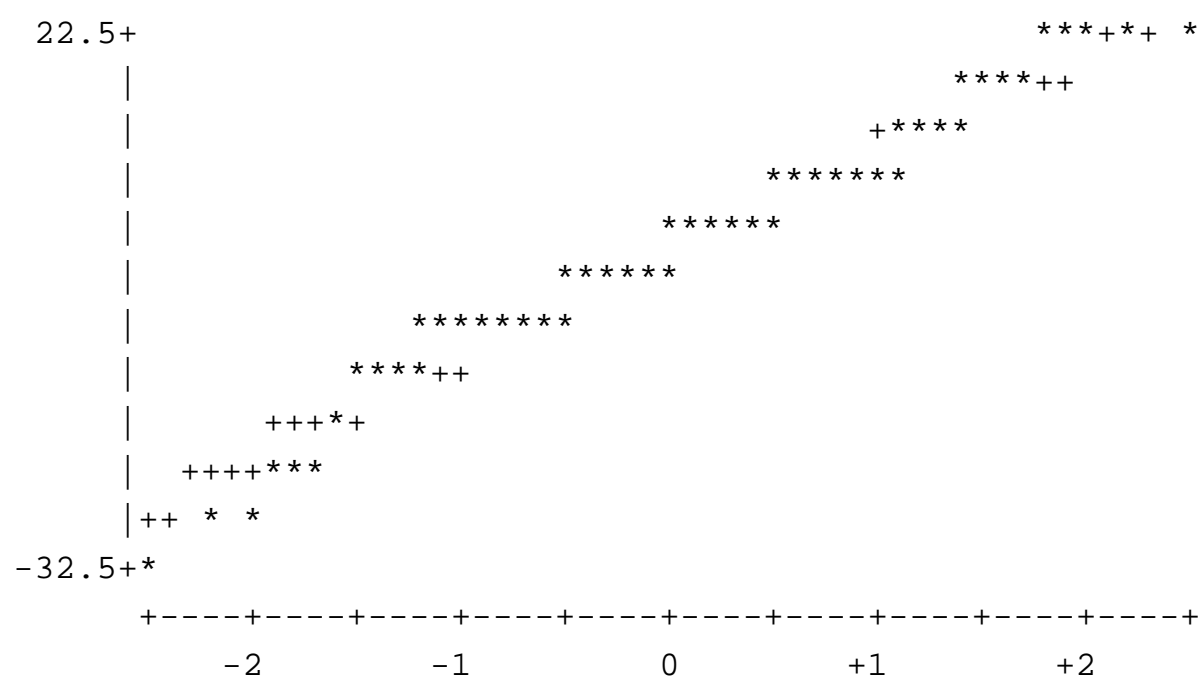

Figure 4.10: Normal Probability Plot for Variable = Productivity. 


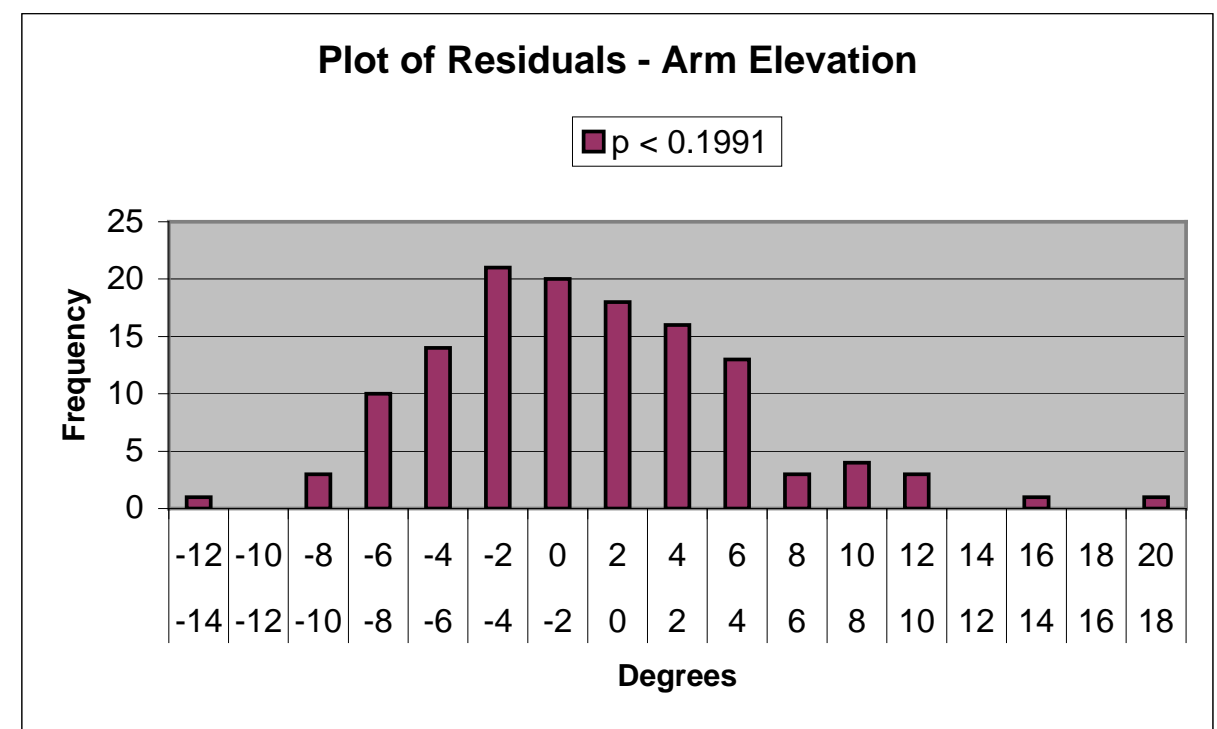

Figure 4.11: Plot of Residuals for Variable = Arm Elevation.

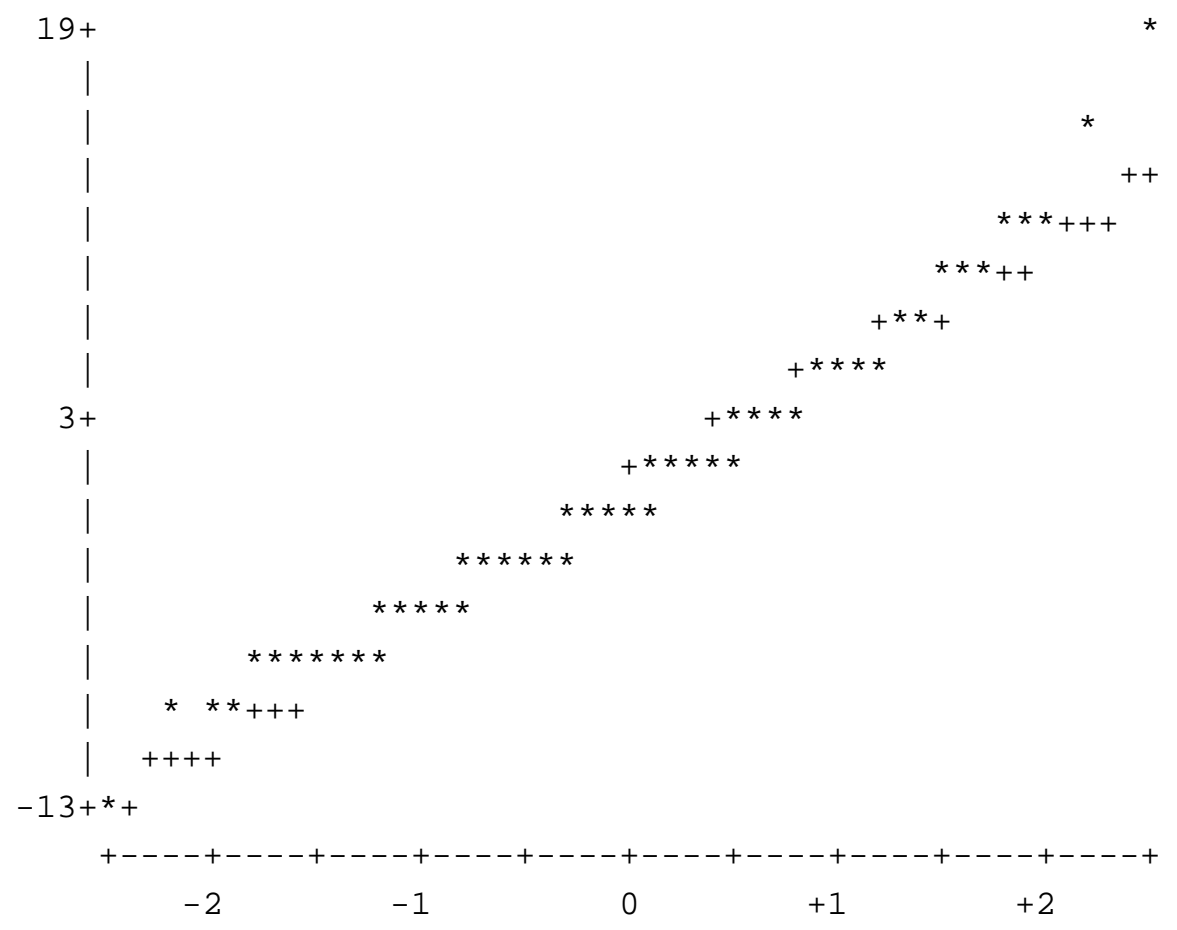

Figure 4.12: Normal Probability Plot for Variable = Arm Elevation. 


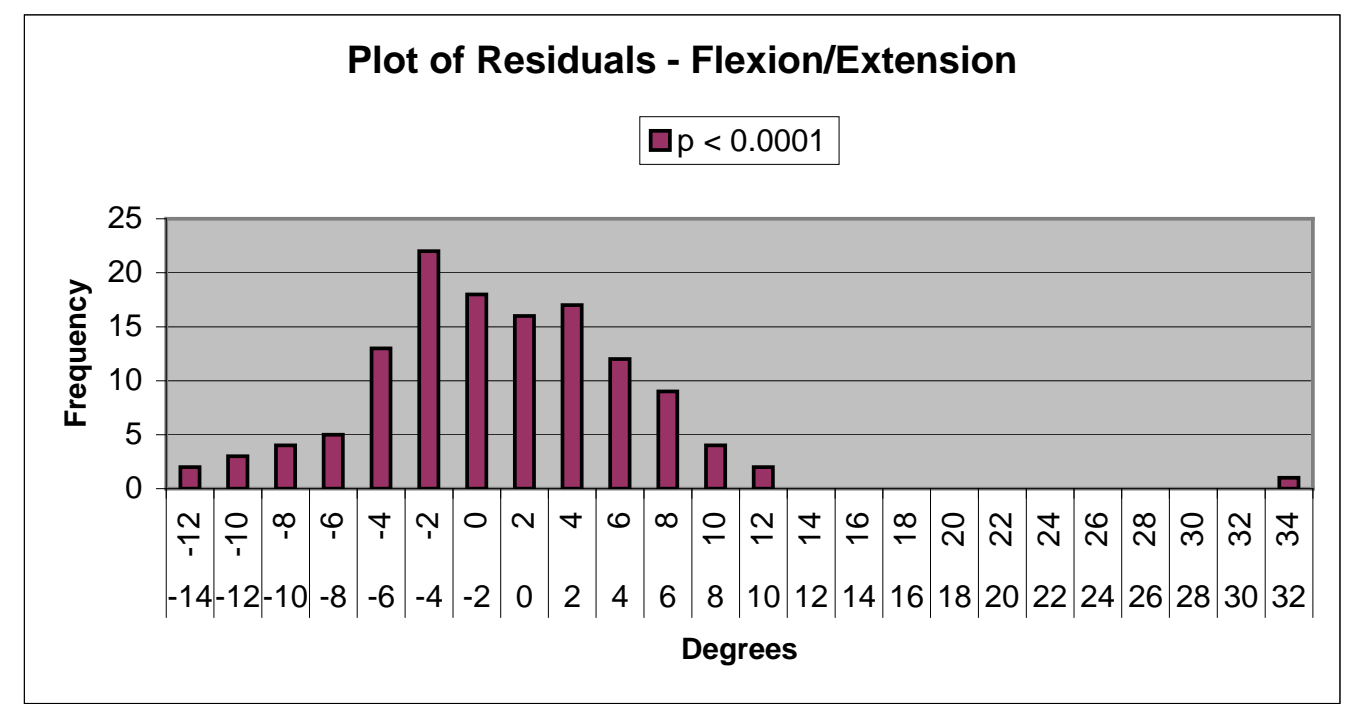

Figure 4.13: Plot of Residuals for Variable $=$ Wrist Flexion/Extension.

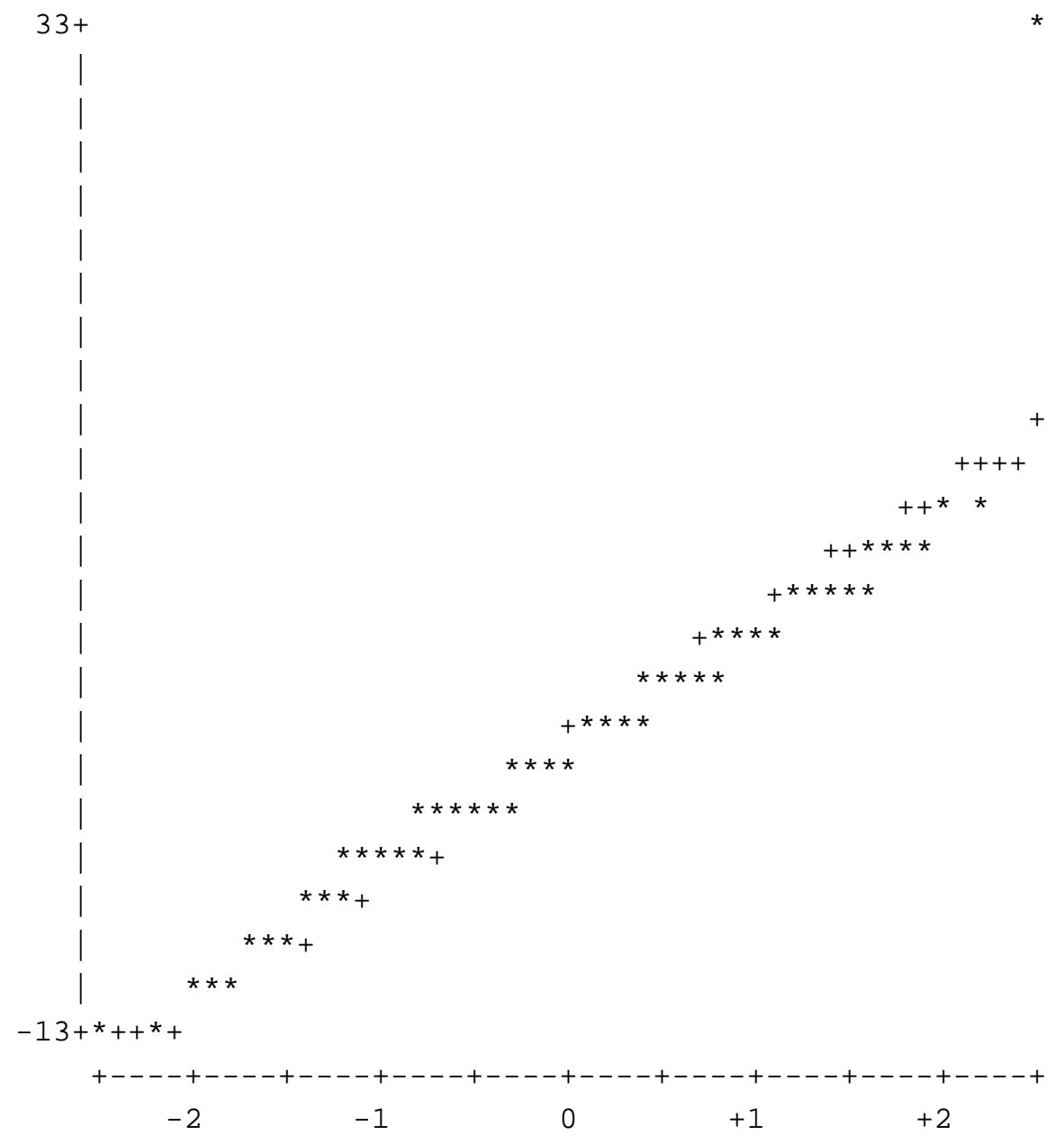

Figure 4.14: Normal Probability Plot for Variable $=$ Wrist Flexion/Extension. 


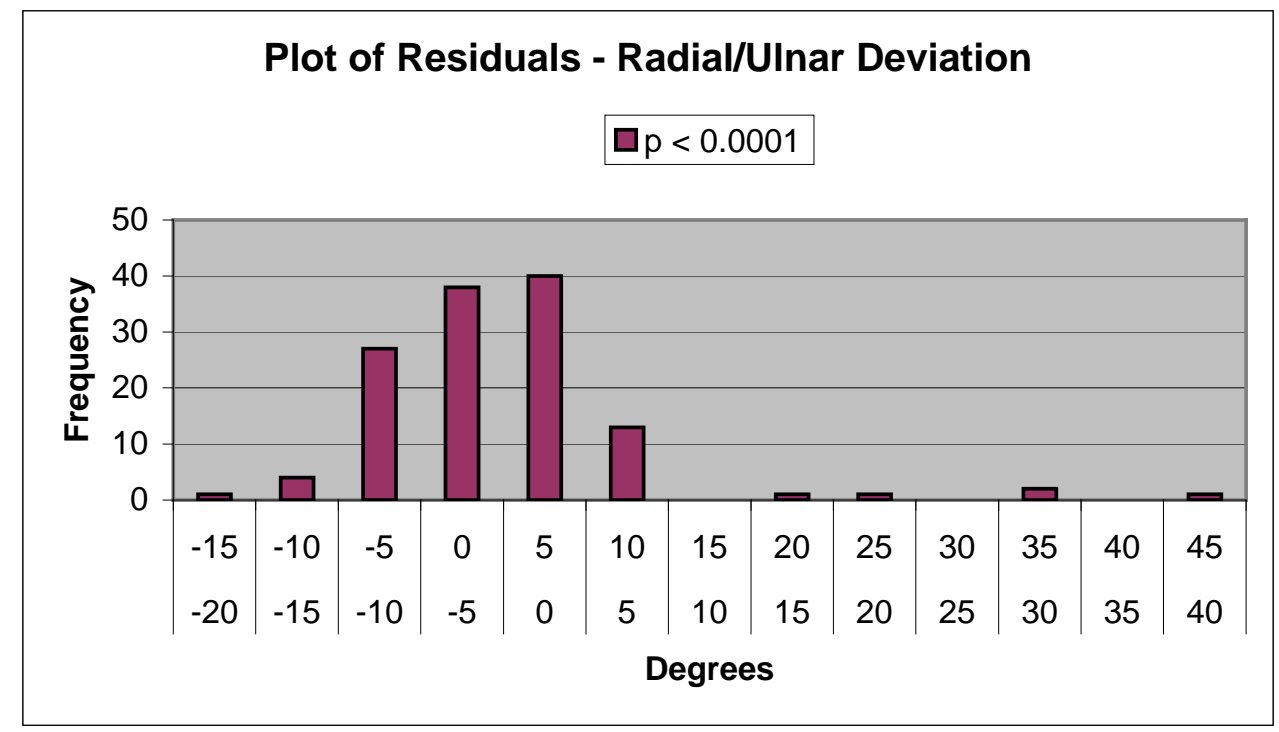

Figure 4.15: Plot of Residuals for Variable $=$ Wrist Radial/Ulnar Deviation.

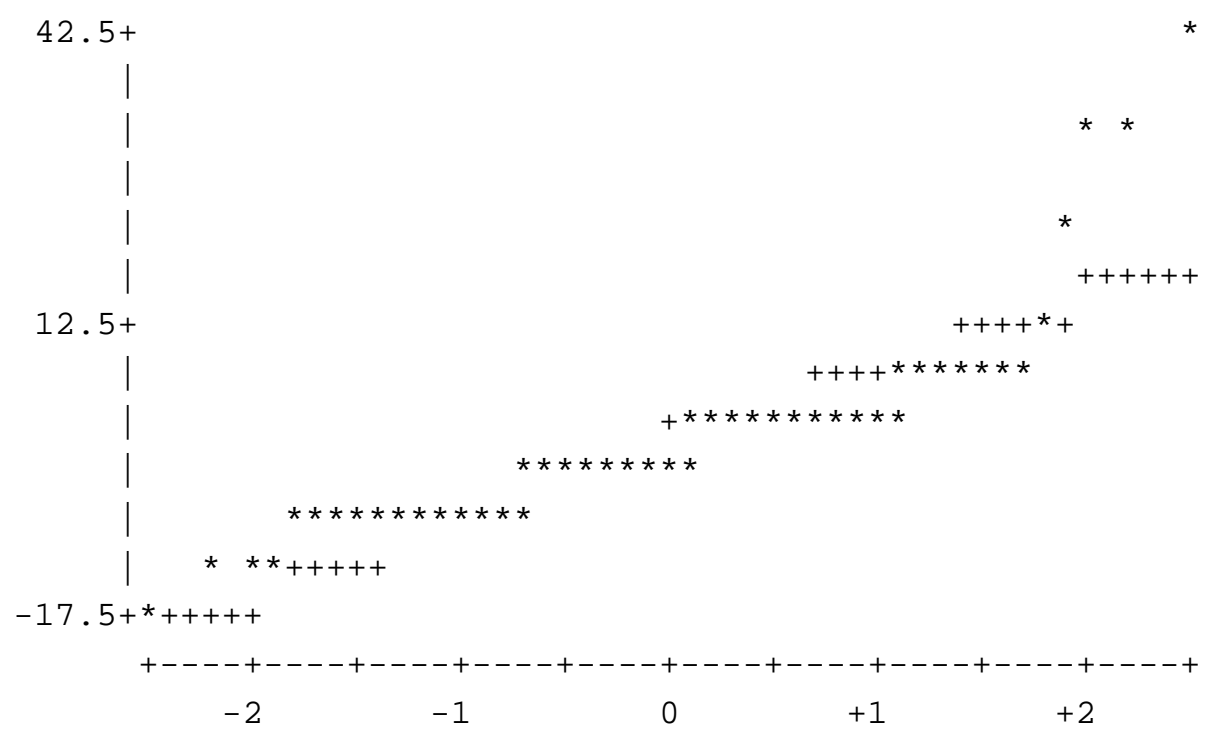

Figure 4.16: Normal Probability Plot for Variable = Radial/Ulnar Deviation 


\subsubsection{Test for Assumption of Equal Variance}

\subsubsection{Jumper Task}

Figures 4.17 through 4.20 show the plots of residual by position in sequence of experimental trials (jumper task), investigating equality of variance for the dependent variables.

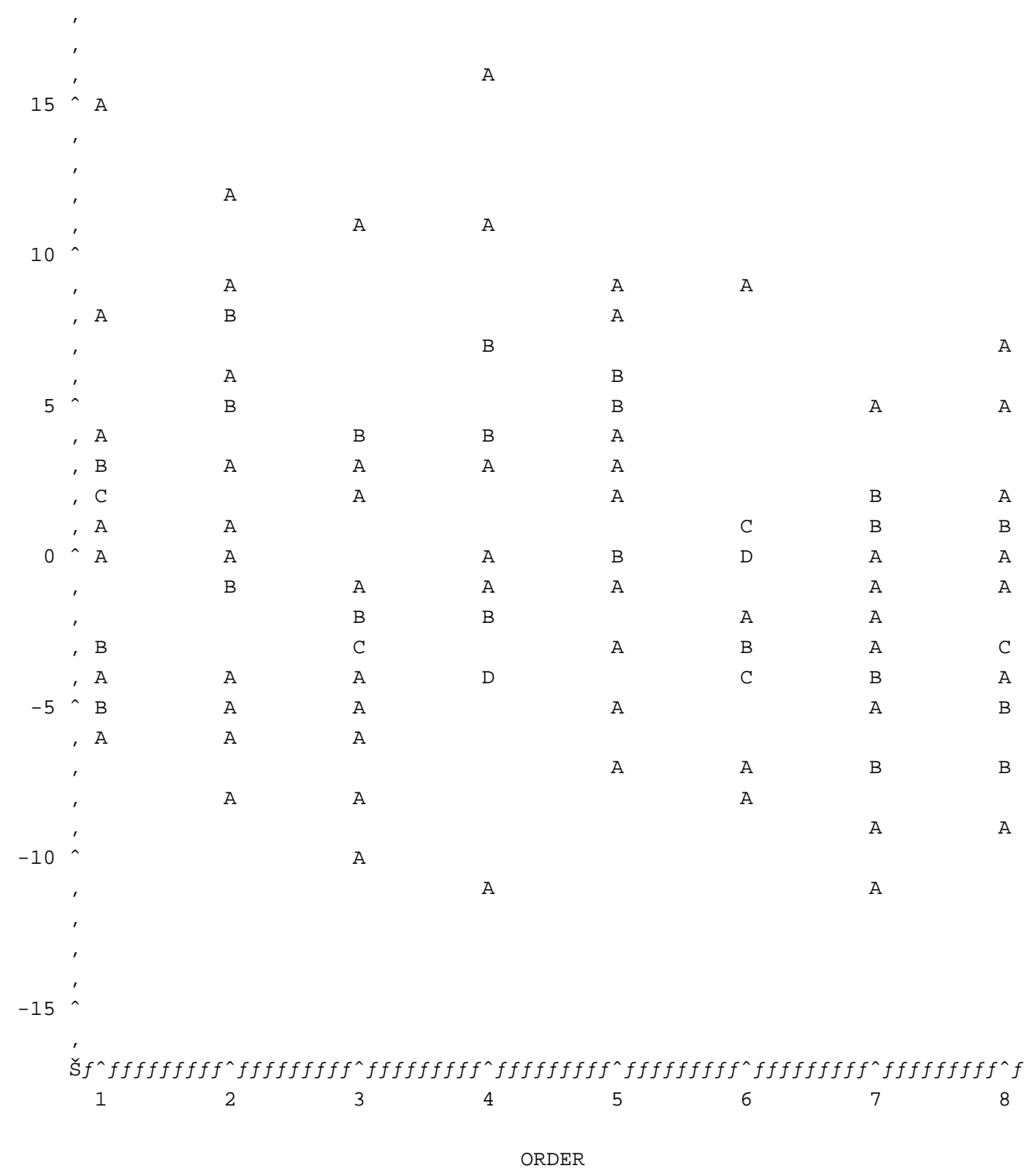

Figure 4.17: Time Order Plot for Productivity Residuals. 
Plot of RARM ELEV ${ }^{\circ}$ ORDER. Legend: $A=1$ obs, $B=2$ obs, etc.

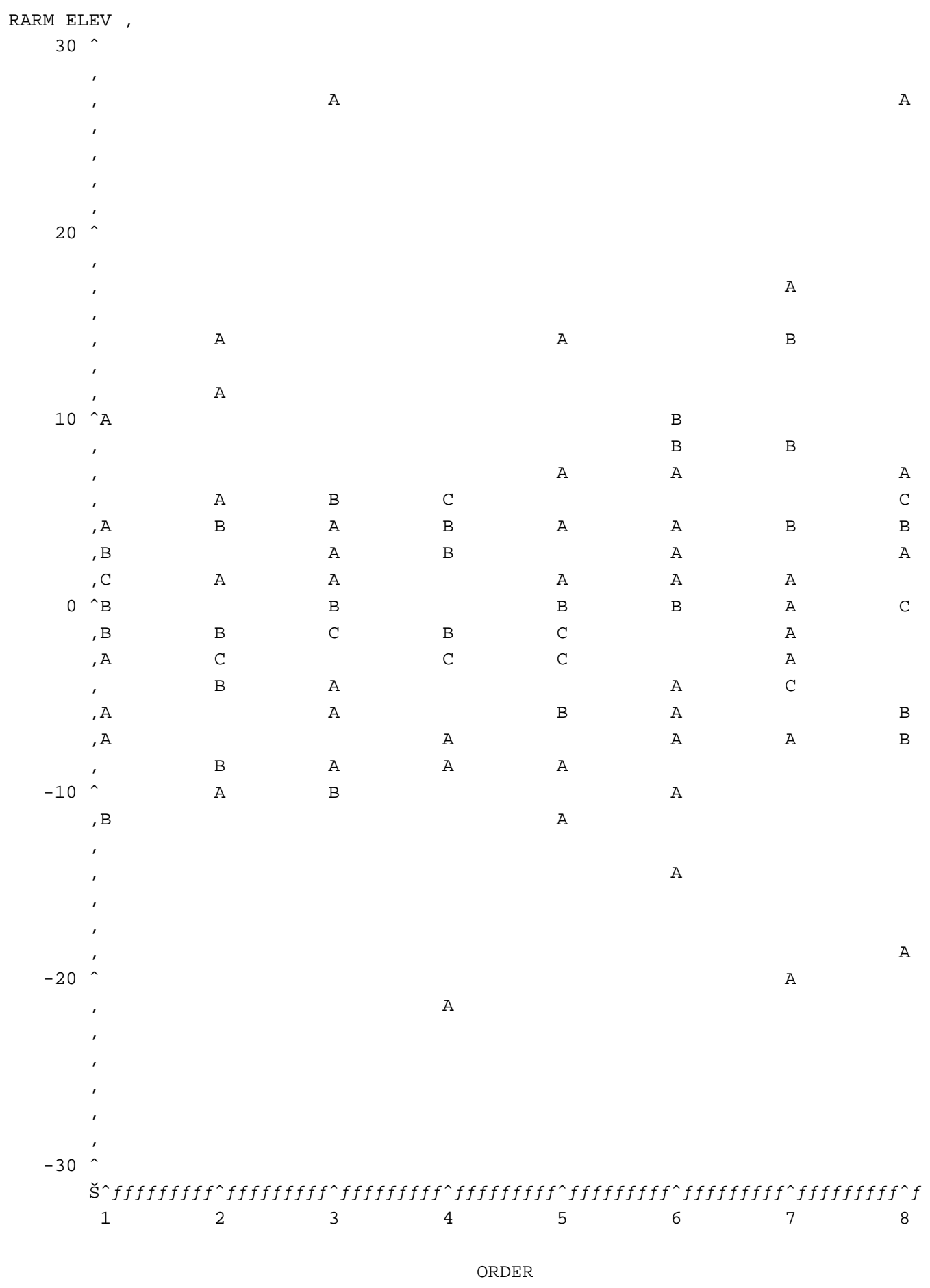

Figure 4.18: Time Order Plot for Arm Elevation Residuals. 
Plot of $\mathrm{RFE}^{\star} \mathrm{ORDER}$. Legend: $\mathrm{A}=1$ obs, $\mathrm{B}=2$ obs, etc.

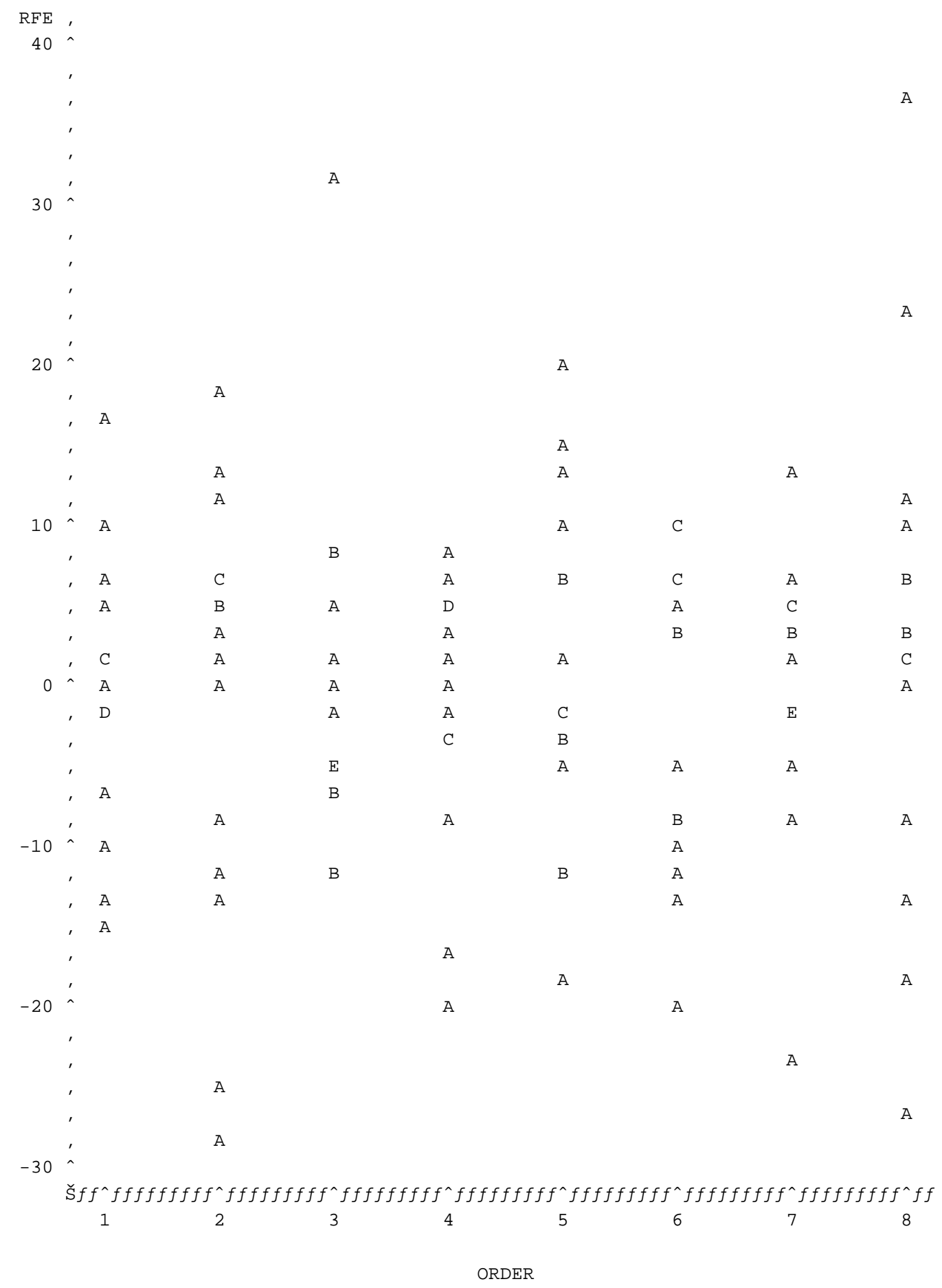

Figure 4.19: Time Order Plot for Flexion/Extension Residuals. 
Plot of RRU*ORDER. Legend: $A=1$ obs, $B=2$ obs, etc.

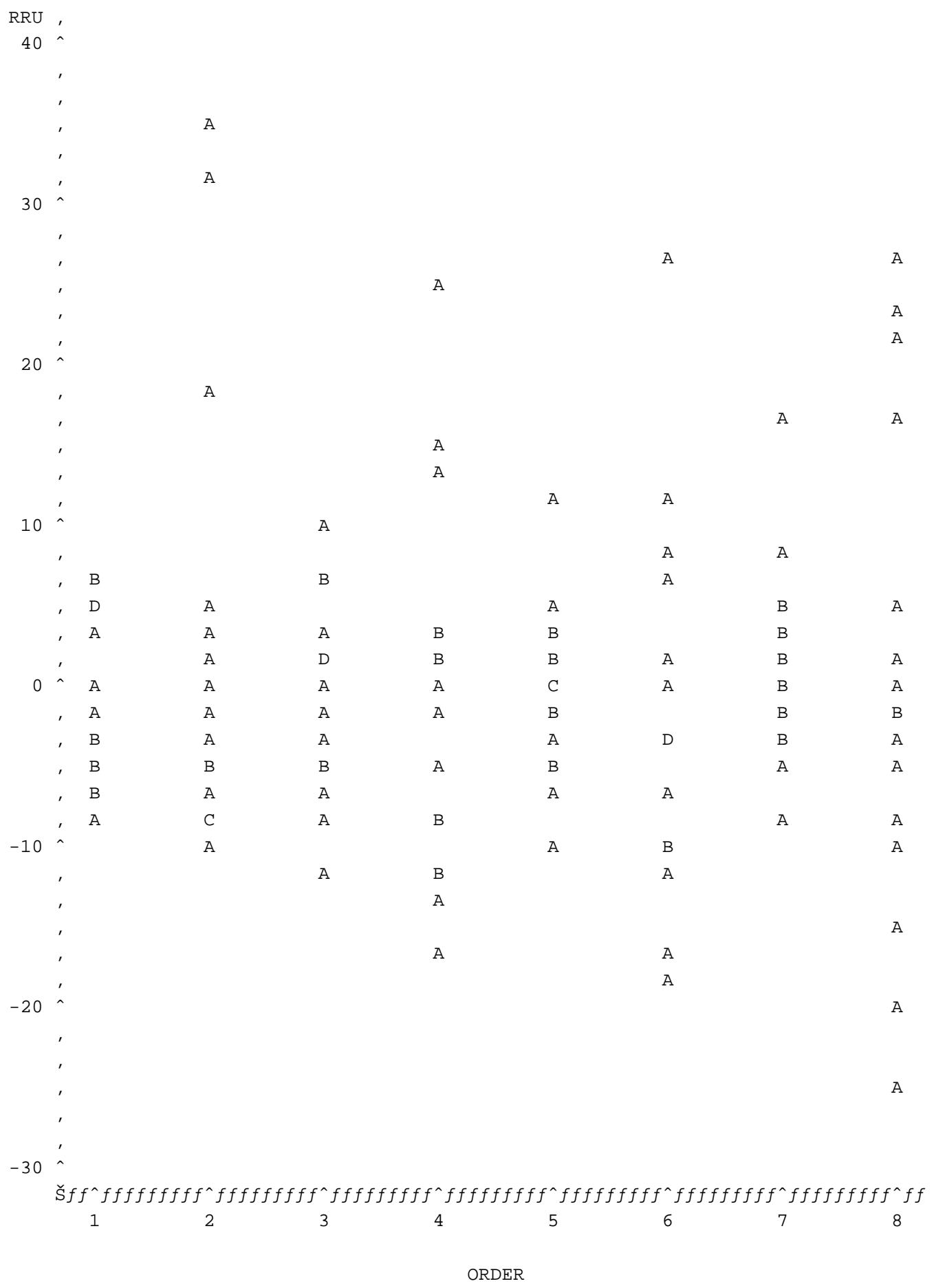

Figure 4.20: Time Order Plot for Radial/Ulnar Deviation Residuals. 


\subsubsection{Spring Task}

Figures 4.21 through 4.24 show the plots of residual by position in sequence of experimental trials (spring task), investigating equality of variance for the dependent variables.

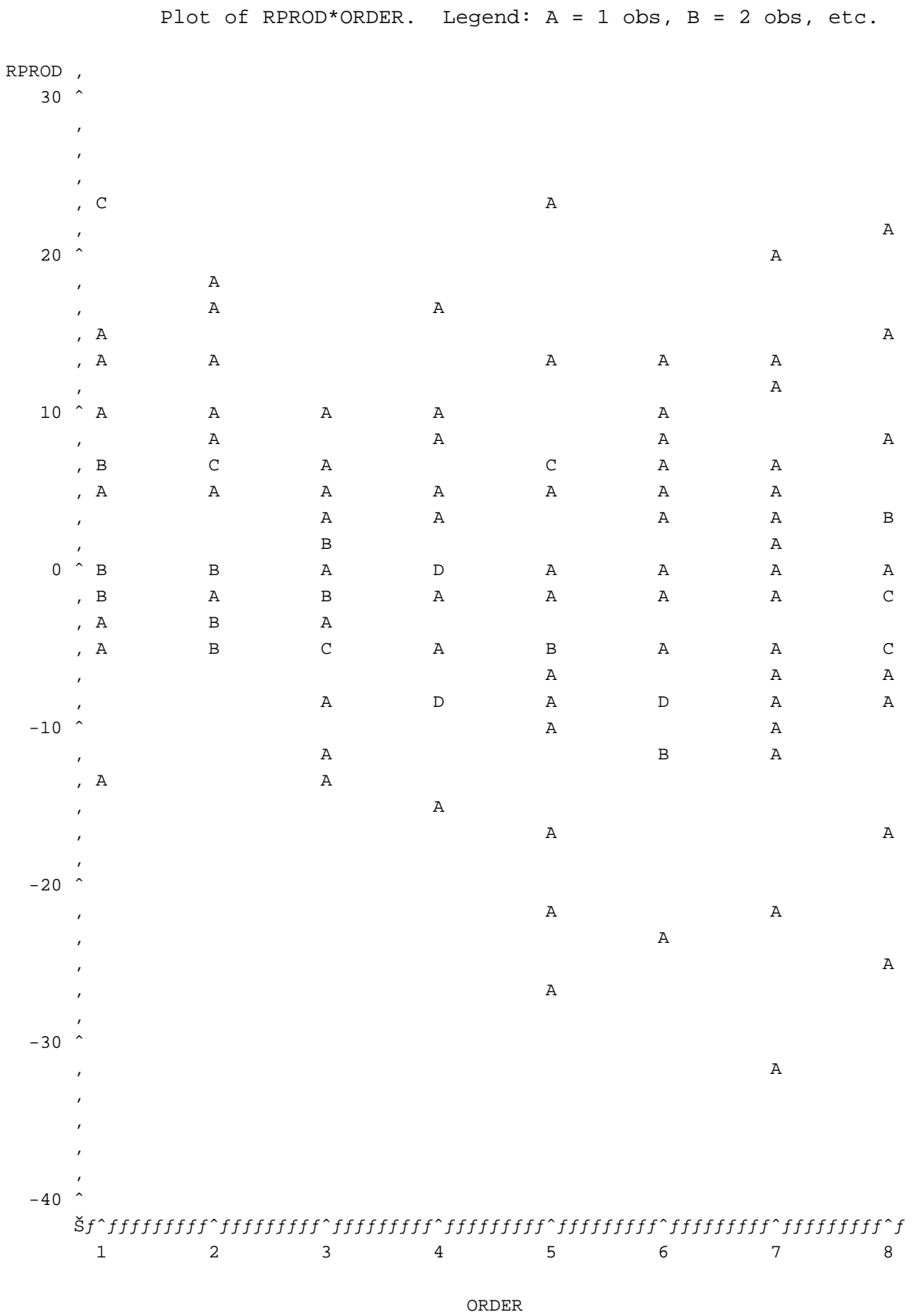

Figure 4.21: Time Order Plot for Productivity Residuals. 
Plot of RARM ELEV*ORDER. Legend: $A=1$ obs, $B=2$ obs, etc.

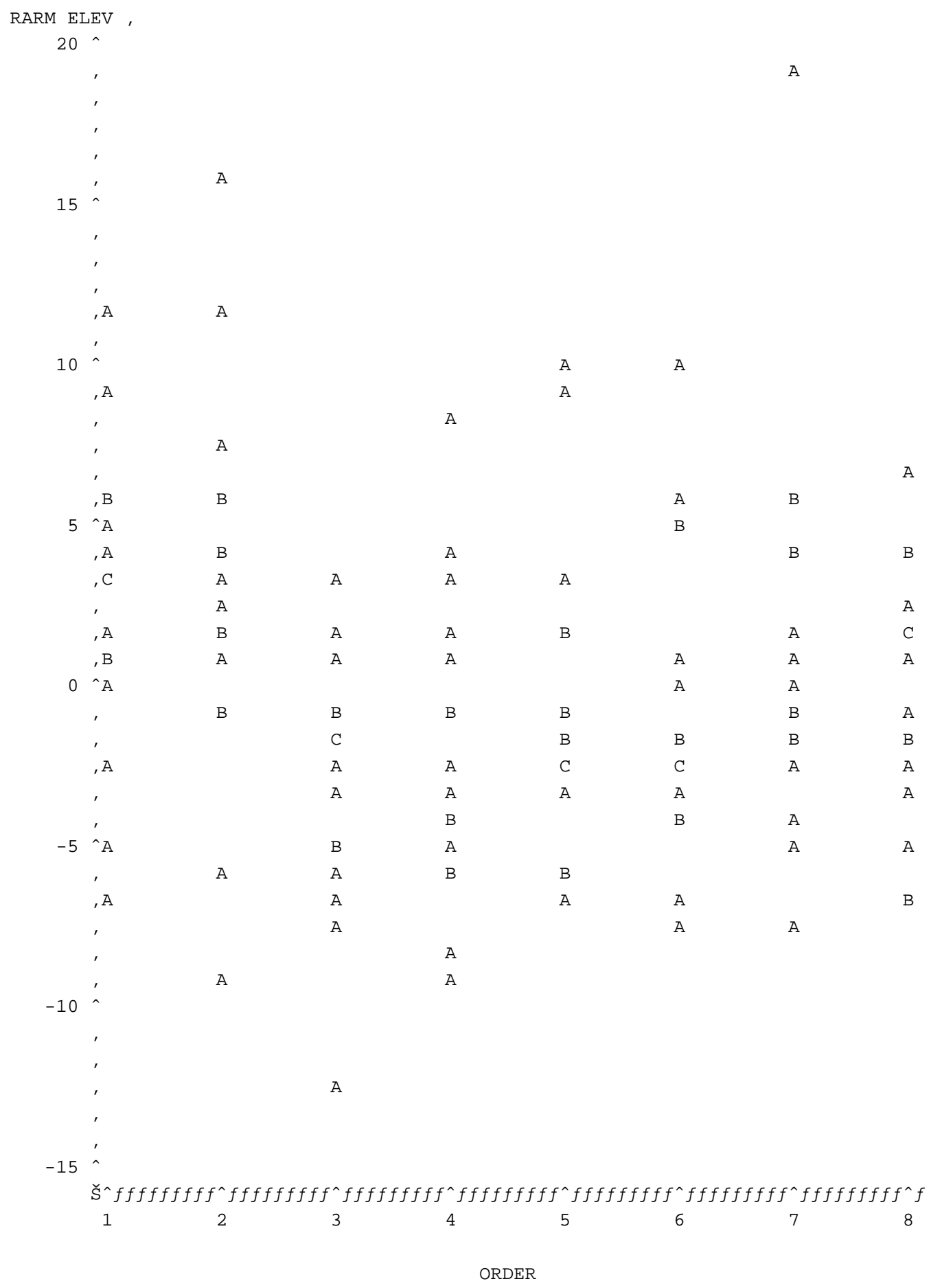

Figure 4.22: Time Order Plot for Arm Elevation Residuals. 
Plot of $\mathrm{RFE}^{\star} \mathrm{ORDER}$. Legend: $\mathrm{A}=1$ obs, $\mathrm{B}=2$ obs, etc.

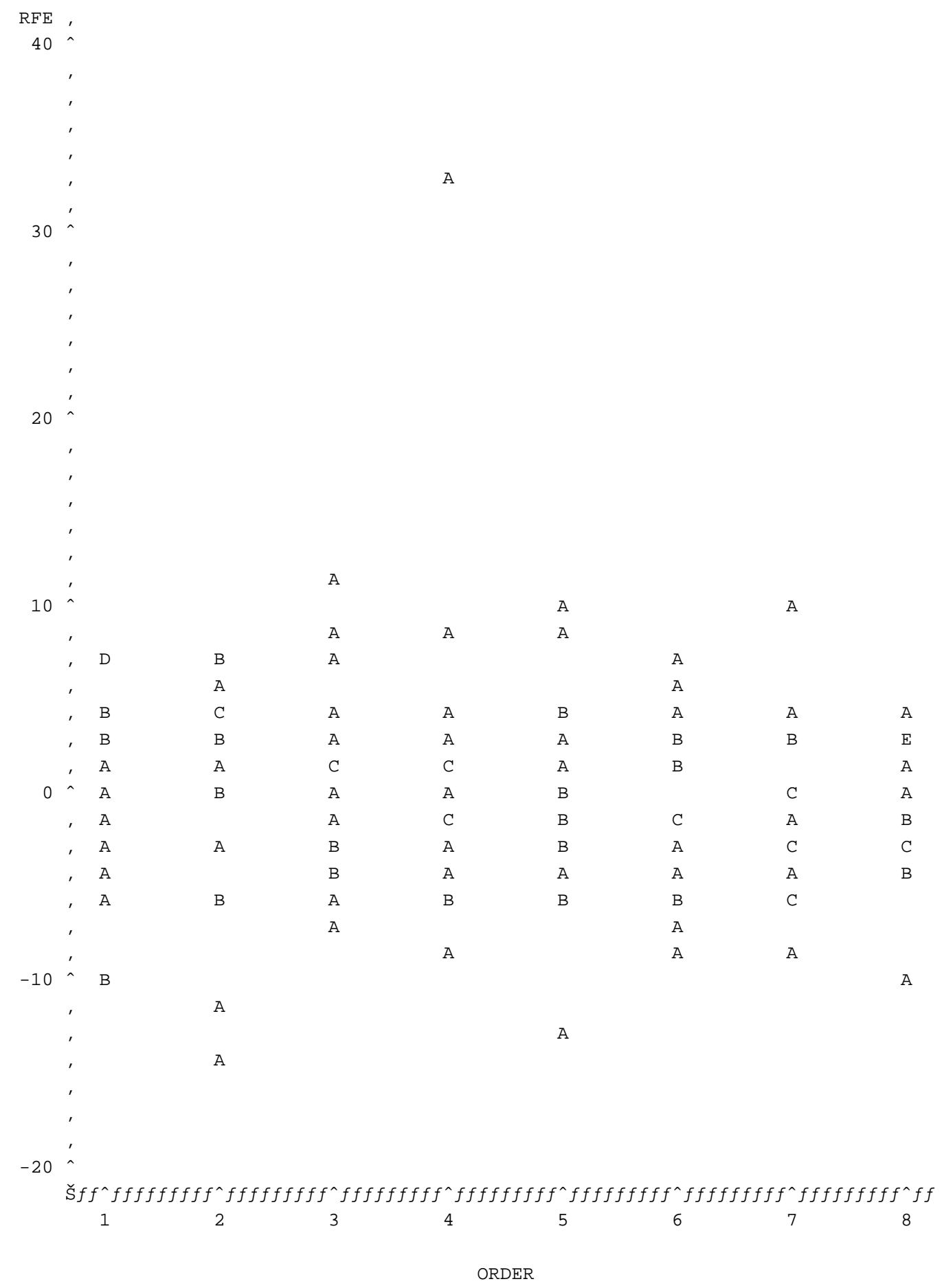

Figure 4.23: Time Order Plot for Flexion/Extension Residuals. 


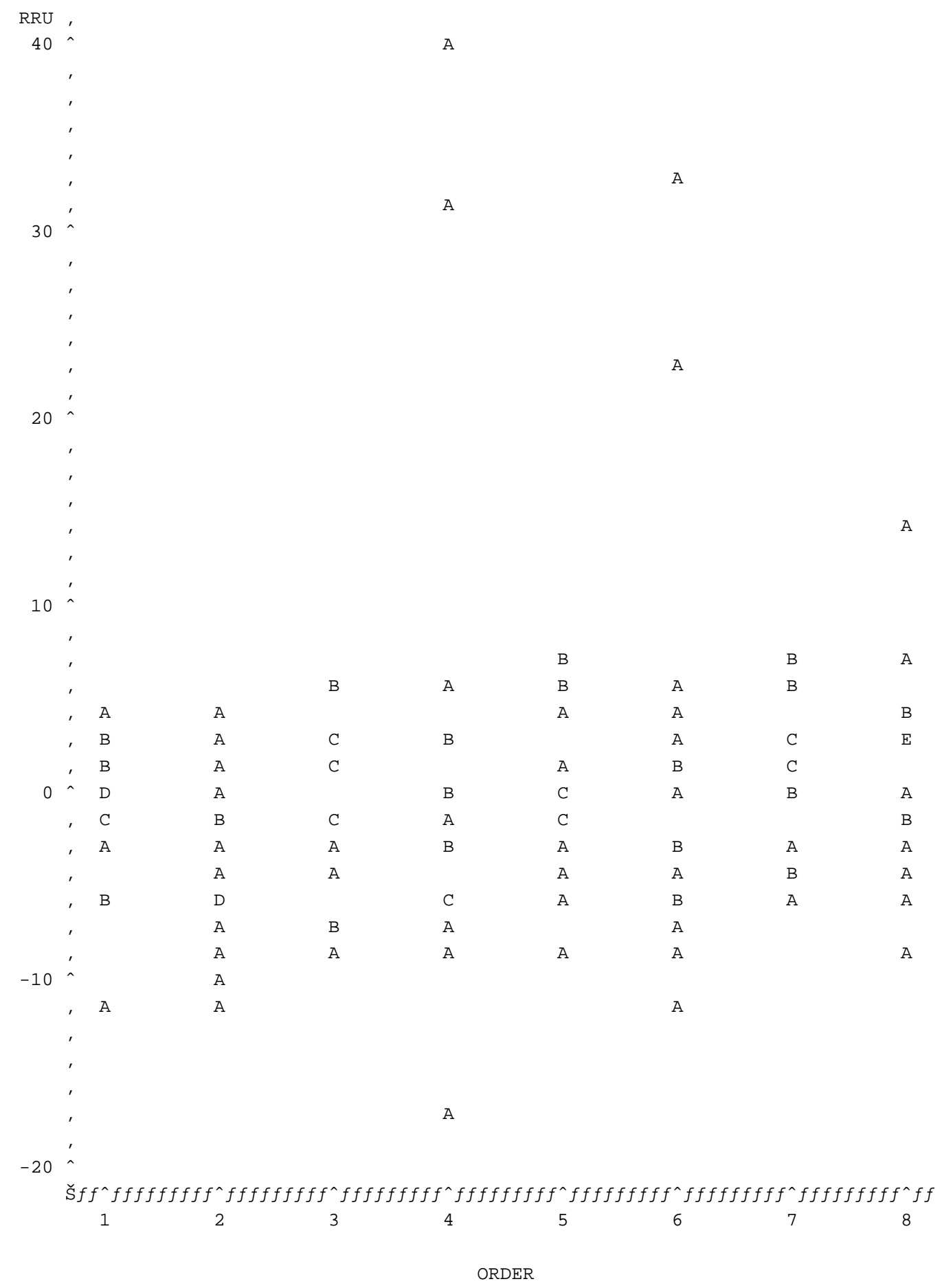

Figure 4.24: Time Order Plot for Radial/Ulnar Deviation Residuals. 
In four cases the results of the Shapiro-Wilk's test indicated rejection of the null hypothesis, and normal distribution of the residuals could not be assumed. In each case outliers were identified from the residuals and were temporarily removed from the data set, and the Shapiro-Wilk's test was run again. In two cases (productivity for the jumper task and flexion/extension for the spring task) the re-run of the test resulted in acceptance of the null hypothesis. Furthermore, analysis of variance of the model with the modified data set provided the same significant effects as the complete data set. In the other two cases (radial/ulnar deviation for both tasks), removal of outliers did not lead to acceptance of the null hypothesis on the Shapiro-Wilk's test. However, analysis of variance was again performed on the modified data set, and in both cases the significant results came out the same as with the complete data set.

To summarize, this preliminary analysis showed that the assumption of equality of variance was satisfied and the departure from normality had no effects on the significant effects. Considering this point, in combination with the fact that the analysis of variance is quite robust to moderate departures of the normality assumption (Montgomery, 1984), the analysis was conducted with the complete data set. Finally, none of the plots of the residuals by positional sequence suggested trends that violated the assumption of equal variance (Montgomery, 1984). 


\subsection{ANOVA Results}

\subsubsection{Jumper Task}

Tables 4.1 through 4.4 list the results on the ANOVA (including F test statistic and P-

value) for the four dependent variables productivity, arm elevation, wrist

flexion/extension, and wrist radial/ulnar deviation and all interactions. (All significant

results - except for Subject - are bolded.)

Table 4.1: Productivity Results.

\begin{tabular}{|l|l|l|}
\hline Effect & F Test Statistic & Prob. $>$ F \\
\hline Subject & 12.13 & P $<0.0001$ \\
\hline Pliers & $\mathbf{1 5 . 2 6}$ & $\mathbf{P}<\mathbf{0 . 0 0 0 2}$ \\
\hline Orientation & $\mathbf{1 3 . 7 3}$ & $\mathbf{P}<\mathbf{0 . 0 0 0 3}$ \\
\hline Constraint & $\mathbf{8 . 5 0}$ & $\mathbf{P}<\mathbf{0 . 0 0 5}$ \\
\hline Pliers x Orientation & 0.03 & not significant \\
\hline Pliers x Constraint & 0.10 & not significant \\
\hline Orientation x Constraint & 0.04 & not significant \\
\hline Pliers x Orientation x Constraint & 1.77 & not significant \\
\hline
\end{tabular}

Table 4. 2: Arm Elevation Results.

\begin{tabular}{|l|l|l|}
\hline Effect & F Test Statistic & Prob. > F \\
\hline Subject & 3.77 & P $<0.0001$ \\
\hline Pliers & $\mathbf{4 9 . 0 7}$ & P $<\mathbf{0 . 0 0 0 1}$ \\
\hline Orientation & $\mathbf{1 3 . 9 0}$ & $\mathbf{P}<\mathbf{0 . 0 0 0 3}$ \\
\hline Constraint & $\mathbf{2 6 . 8 4}$ & $\mathbf{P}<\mathbf{0 . 0 0 0 1}$ \\
\hline Pliers x Orientation & $\mathbf{5 . 5 6}$ & $\mathbf{P}<\mathbf{0 . 0 3}$ \\
\hline Pliers x Constraint & $\mathbf{2 7 . 1 5}$ & $\mathbf{P}<\mathbf{0 . 0 0 0 1}$ \\
\hline Orientation x Constraint & $\mathbf{1 0 . 4 9}$ & $\mathbf{P}<\mathbf{0 . 0 0 2}$ \\
\hline Pliers x Orientation x Constraint & 3.78 & P $<0.0545$ \\
\hline
\end{tabular}


Table 4.3: Wrist Flexion/Extension Results.

\begin{tabular}{|l|l|l|}
\hline Effect & F Test Statistic & Prob. $>$ F \\
\hline Subject & 4.81 & P $<0.0001$ \\
\hline Pliers & 1.89 & not significant \\
\hline Orientation & 0.03 & not significant \\
\hline Constraint & $\mathbf{2 4 . 7 0}$ & $\mathbf{P}<\mathbf{0 . 0 0 0 1}$ \\
\hline Pliers x Orientation & $\mathbf{5 . 4 9}$ & $\mathbf{P}<\mathbf{0 . 0 3}$ \\
\hline Pliers x Constraint & $\mathbf{1 0 . 1 6}$ & $\mathbf{P}<\mathbf{0 . 0 0 2}$ \\
\hline Orientation x Constraint & $\mathbf{4 . 3 3}$ & $\mathbf{P}<\mathbf{0 . 0 4}$ \\
\hline Pliers x Orientation x Constraint & 0.58 & not significant \\
\hline
\end{tabular}

Table 4.4: Wrist Radial/Ulnar Deviation Results.

\begin{tabular}{|l|l|l|}
\hline Effect & F Test Statistic & Prob. $>$ F \\
\hline Subject & 4.71 & P $<0.0001$ \\
\hline Pliers & 1.87 & not significant \\
\hline Orientation & 2.81 & not significant \\
\hline Constraint & $\mathbf{2 6 . 9 9}$ & $\mathbf{P}<\mathbf{0 . 0 0 0 1}$ \\
\hline Pliers x Orientation & $\mathbf{1 1 . 3 1}$ & $\mathbf{P}<\mathbf{0 . 0 0 2}$ \\
\hline Pliers x Constraint & $\mathbf{9 . 7 2}$ & $\mathbf{P}<\mathbf{0 . 0 0 3}$ \\
\hline Orientation x Constraint & $\mathbf{7 . 5 1}$ & $\mathbf{P}<\mathbf{0 . 0 1}$ \\
\hline Pliers x Orientation x Constraint & 1.33 & not significant \\
\hline
\end{tabular}


Figures 4.25 through 4.33 show the significant two-way interaction effects for each of the dependent variables (jumper task).

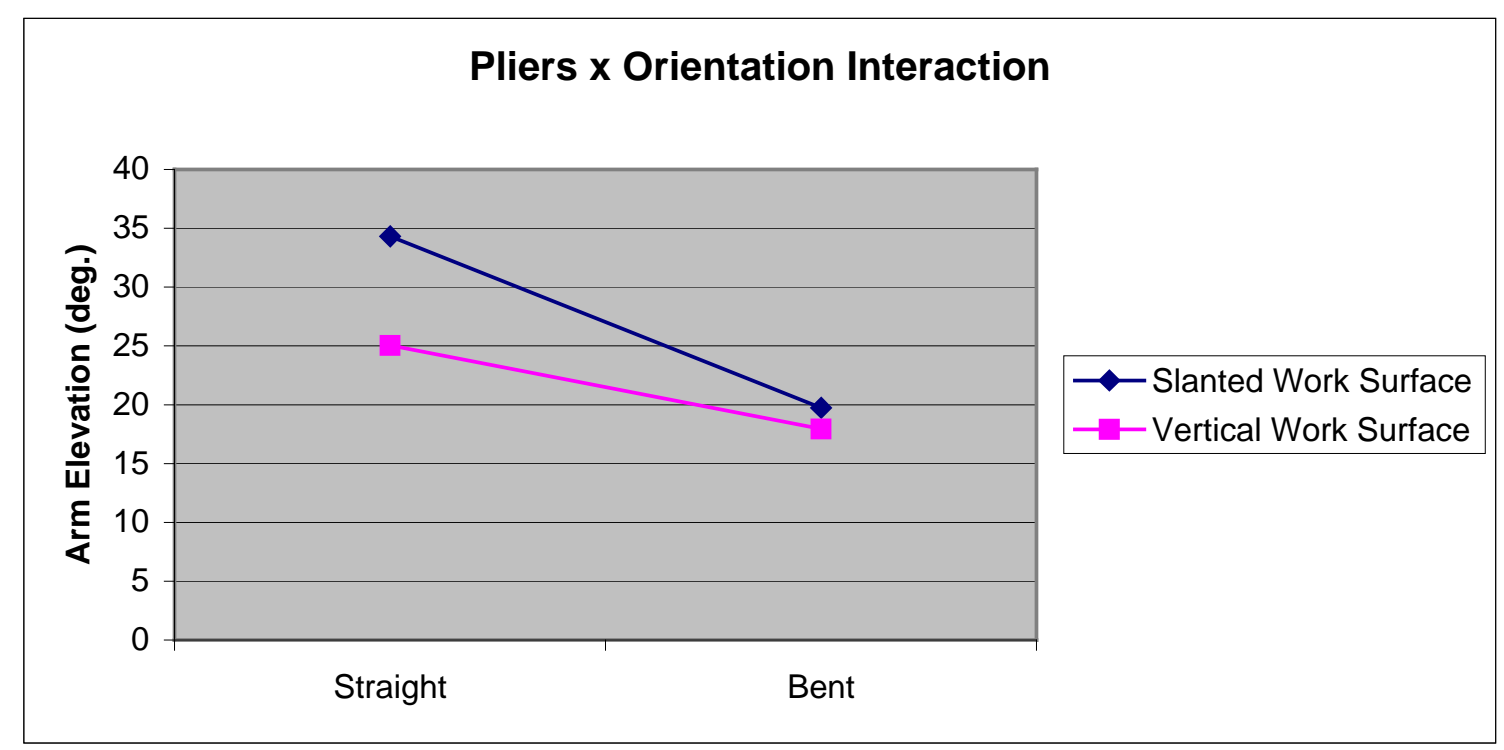

Figure 4.25: Interaction Effect of Pliers x Orientation on Arm Elevation.

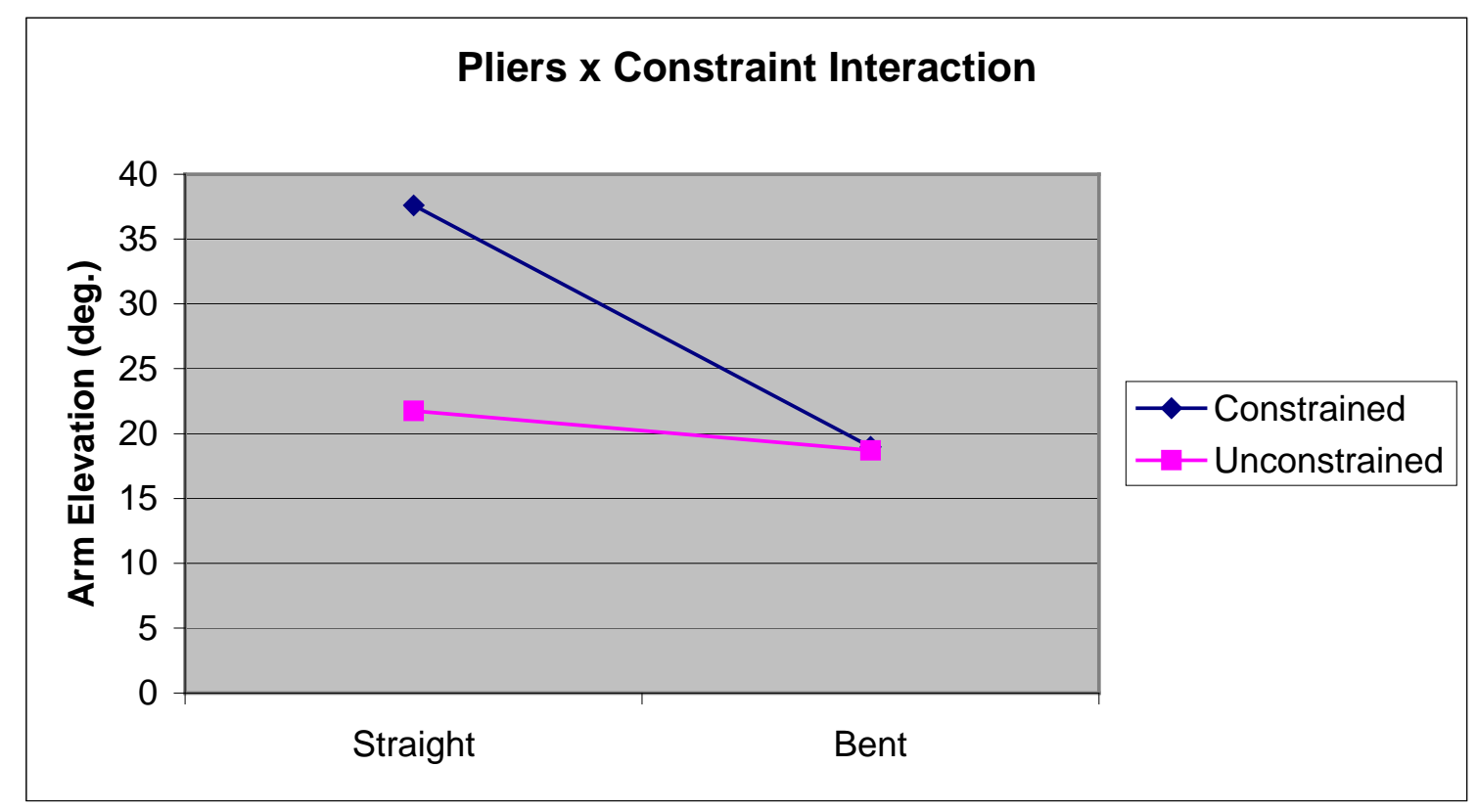

Figure 4.26: Interaction Effect of Pliers x Constraint on Arm Elevation. 


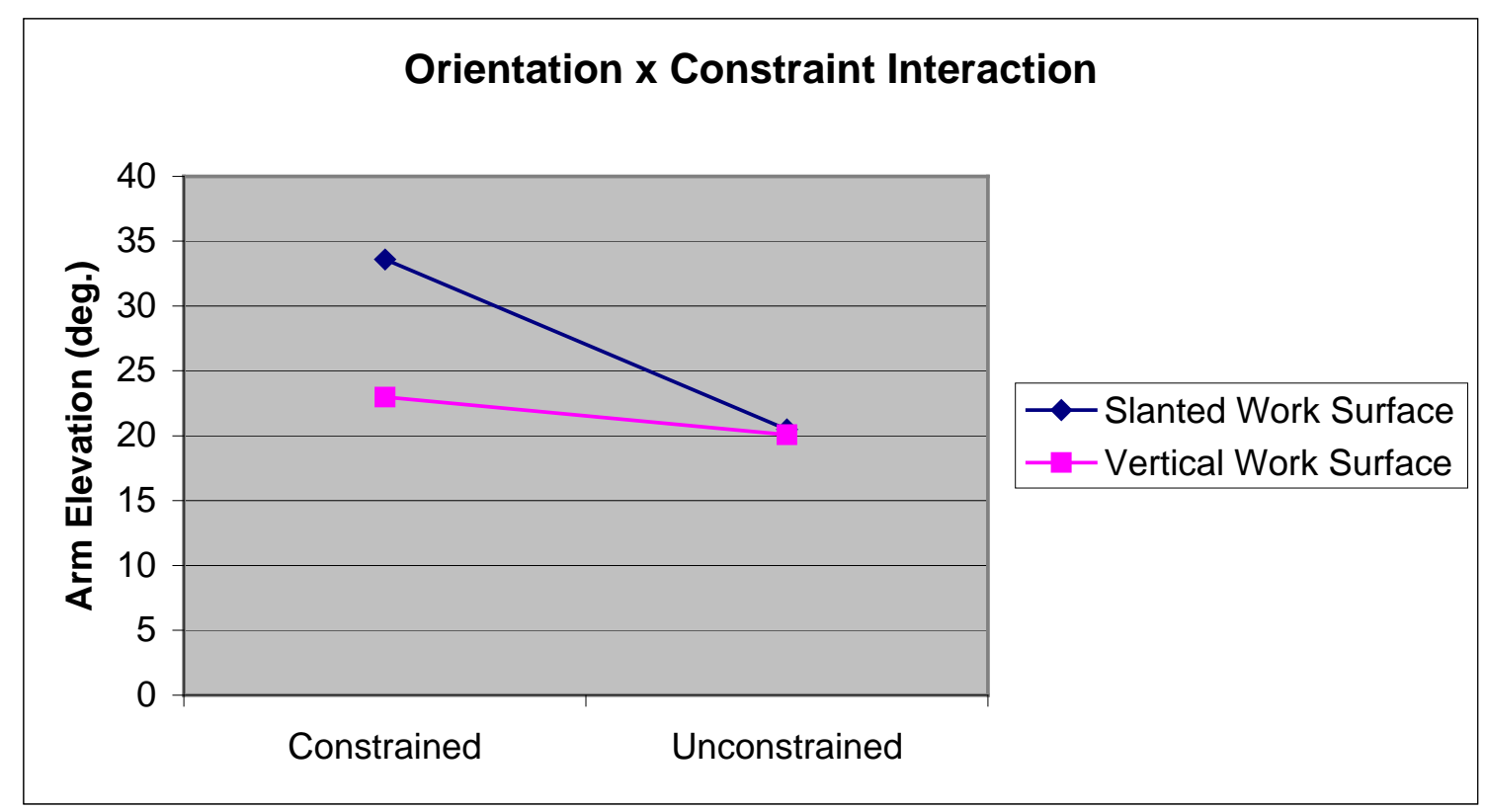

Figure 4.27: Interaction Effect of Orientation x Constraint on Arm Elevation.

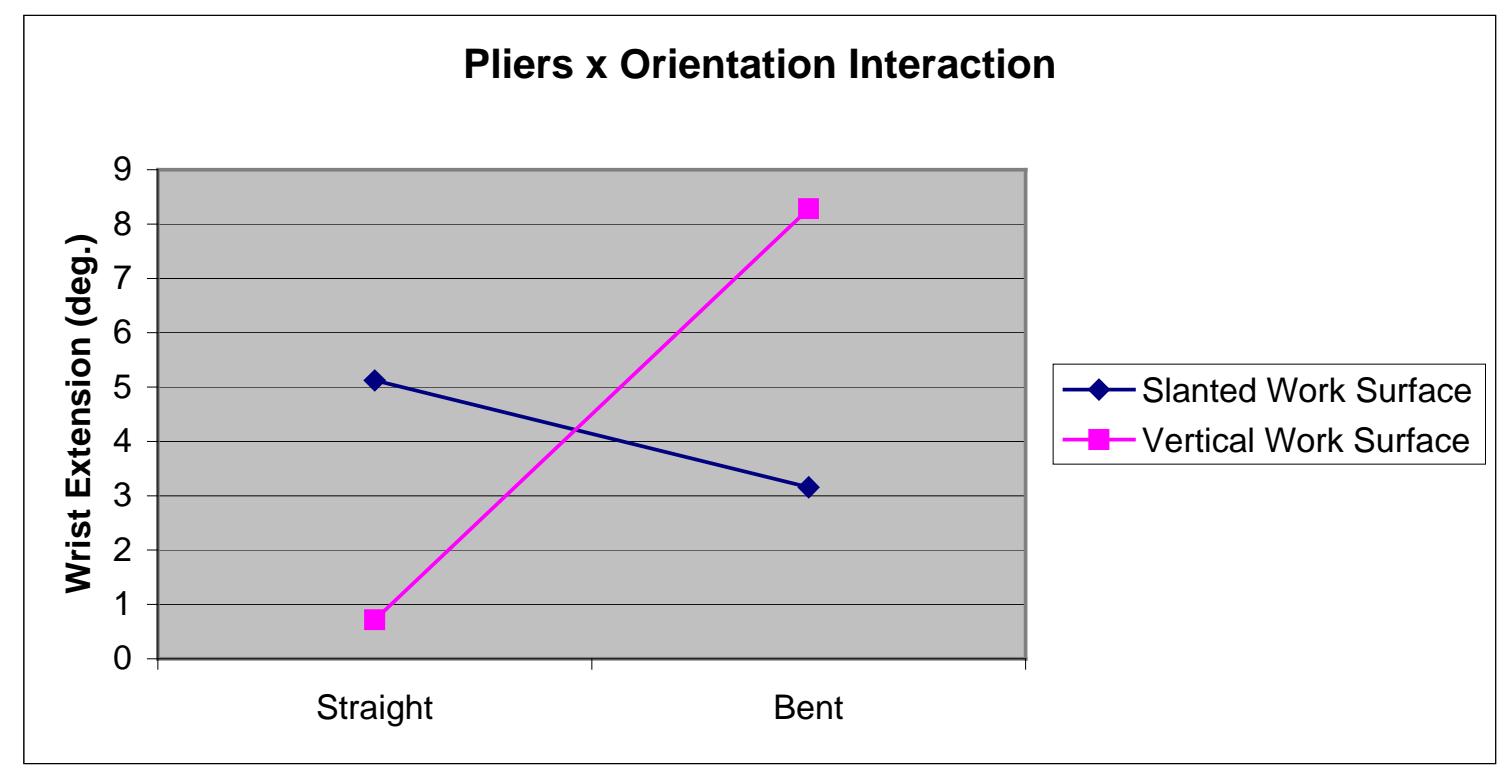

Figure 4.28: Interaction Effect of Pliers x Orientation on Wrist Extension. 


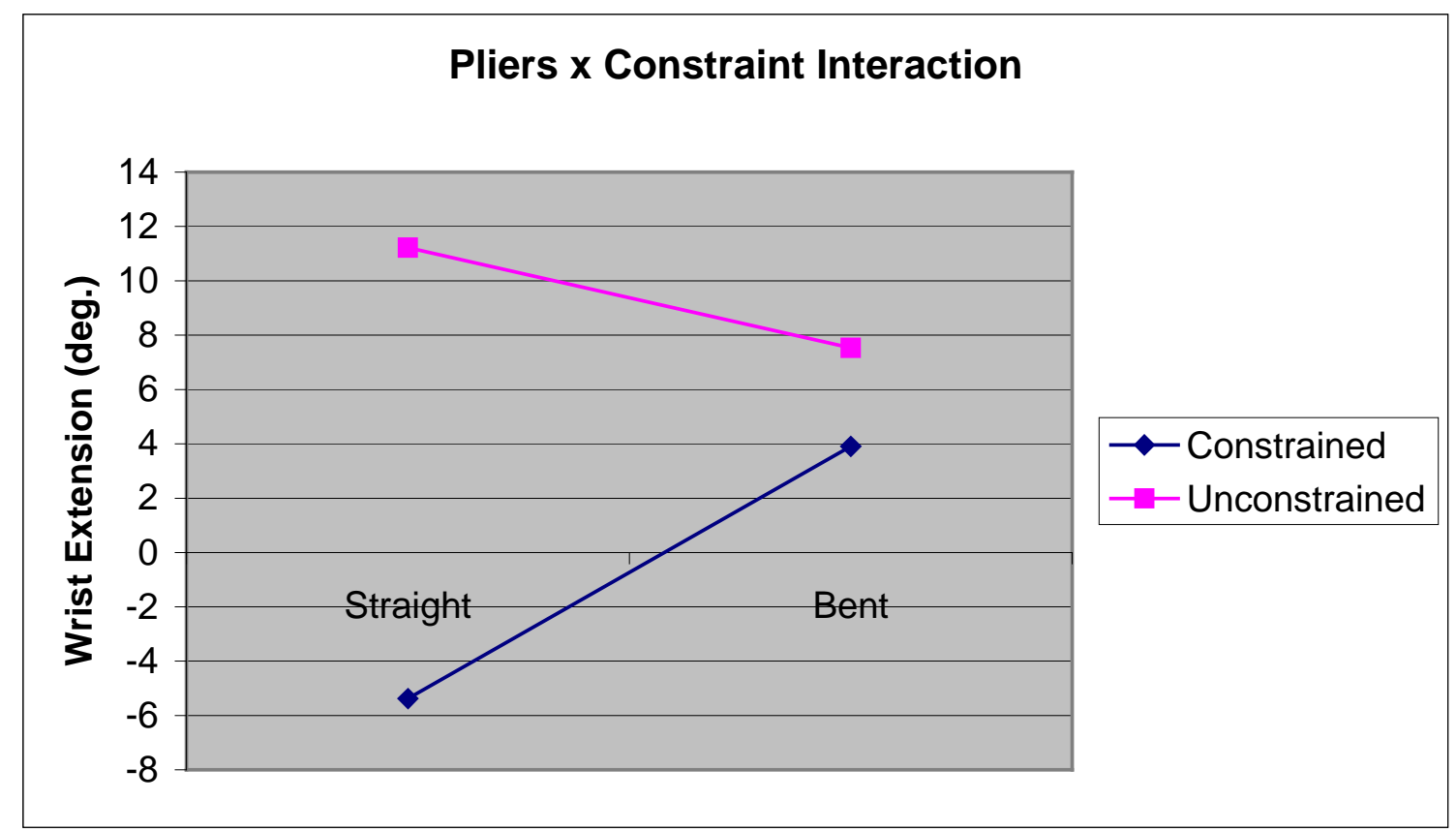

Figure 4.29: Interaction Effect of Pliers x Constraint on Wrist Extension.

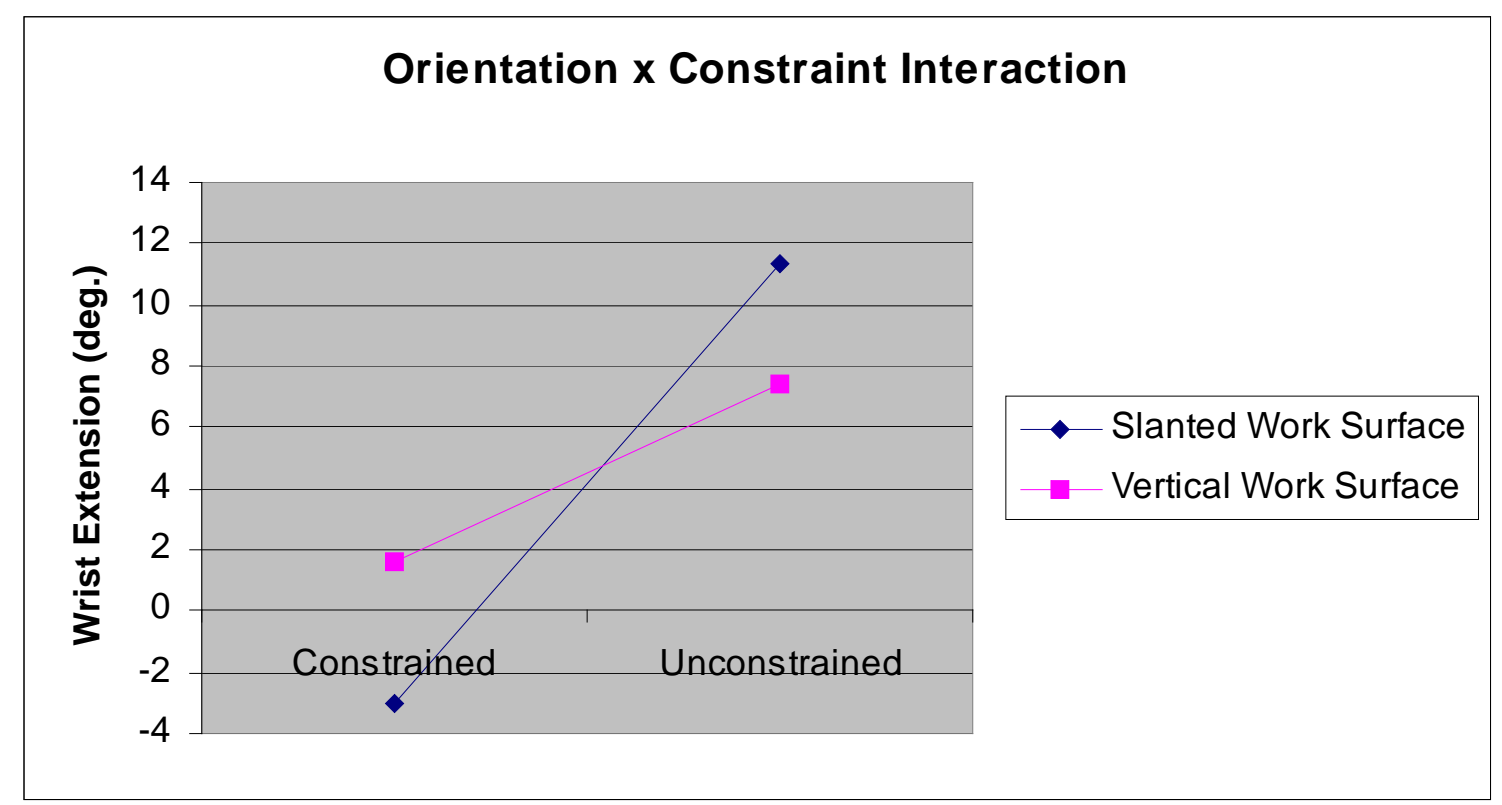

Figure 4.30: Interaction Effect of Orientation x Constraint on Wrist Extension. 


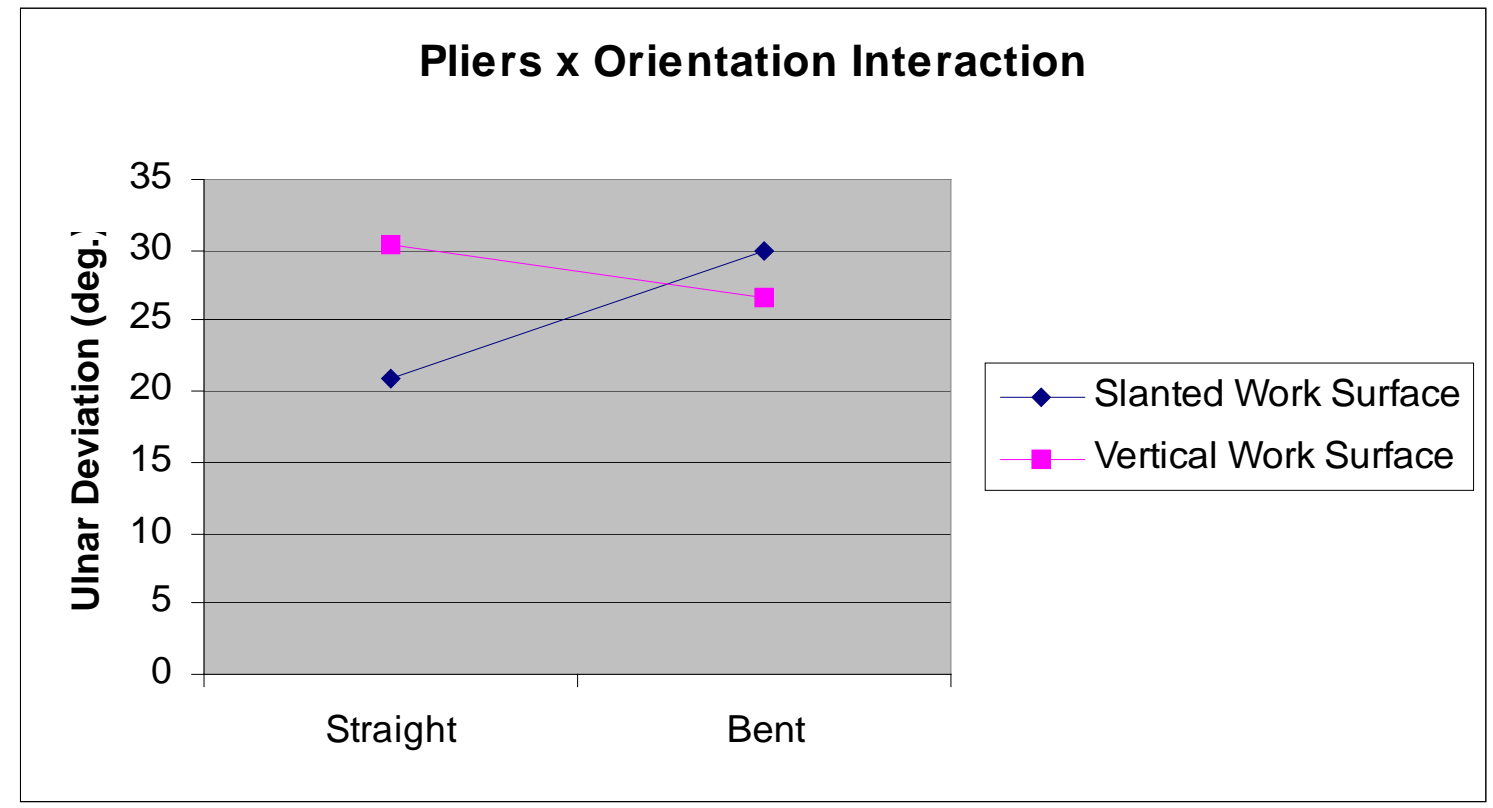

Figure 4.31: Interaction Effect of Pliers x Orientation on Ulnar Deviation.

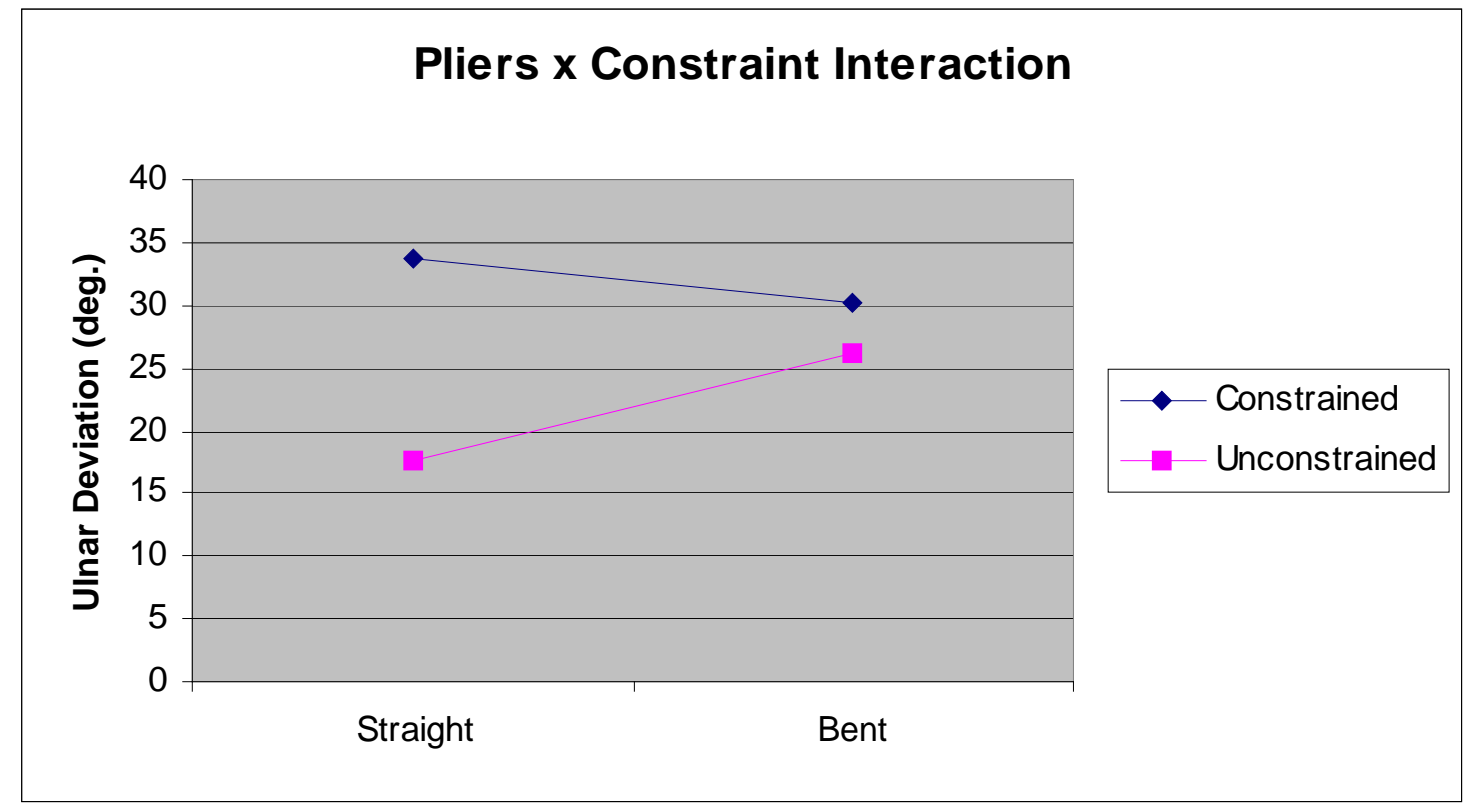

Figure 4.32: Interaction Effect of Pliers x Constraint on Ulnar Deviation. 


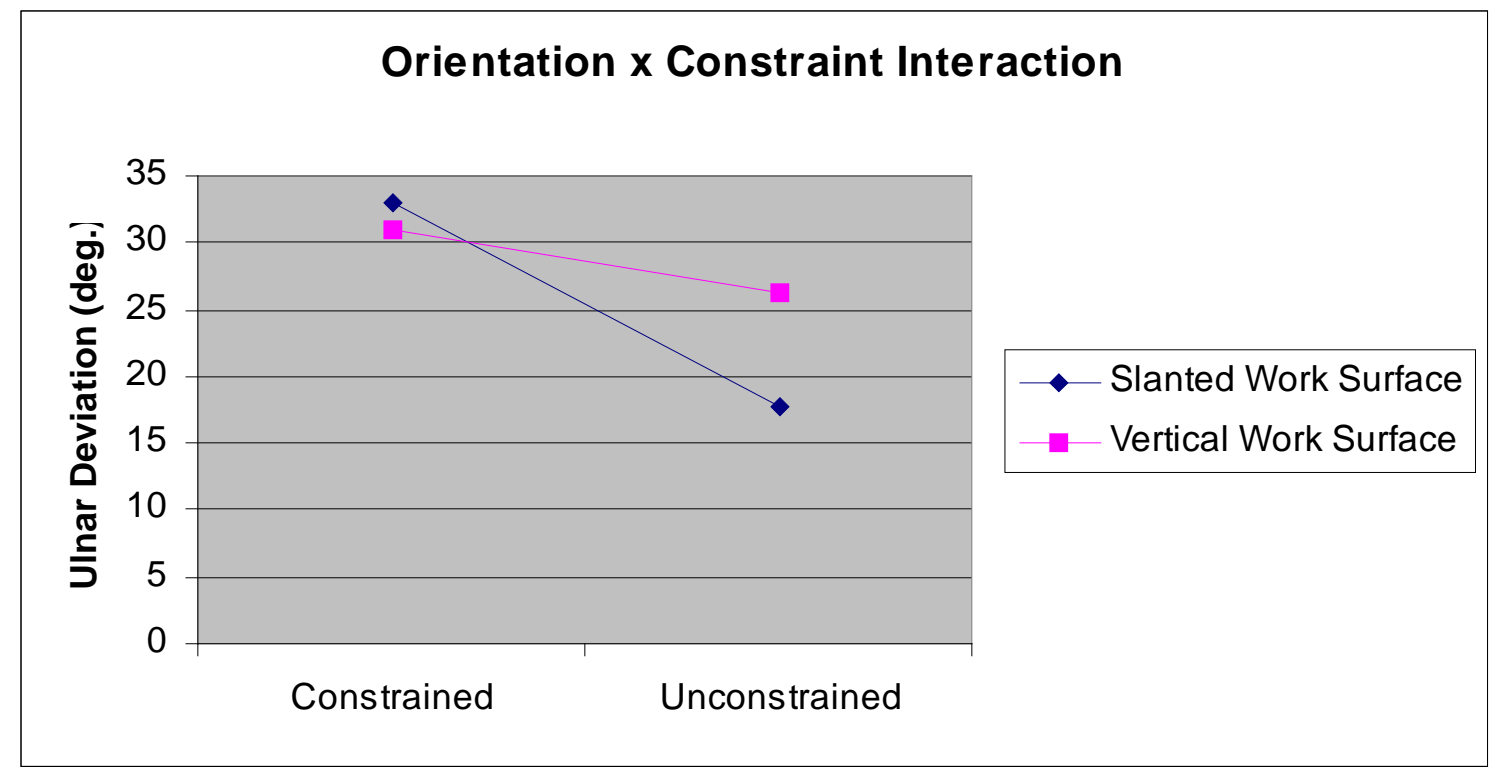

Figure 4.33: Interaction Effect of Orientation x Constraint on Ulnar Deviation. 
Figures 4.34 through 4.37 show the significant main effects for each of the dependent variables (jumper task). Note: significant main effects that were invalidated by a higher order interaction are not plotted.

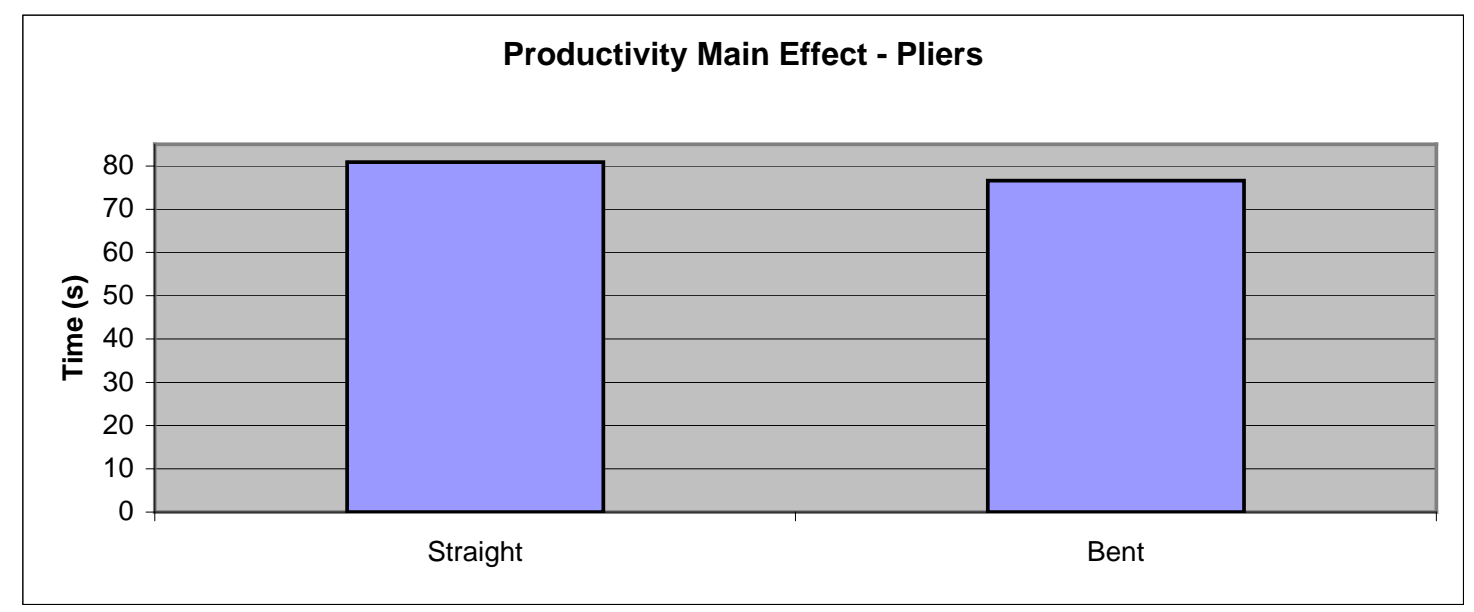

Figure 4.34: Main Effect of Pliers on Productivity.

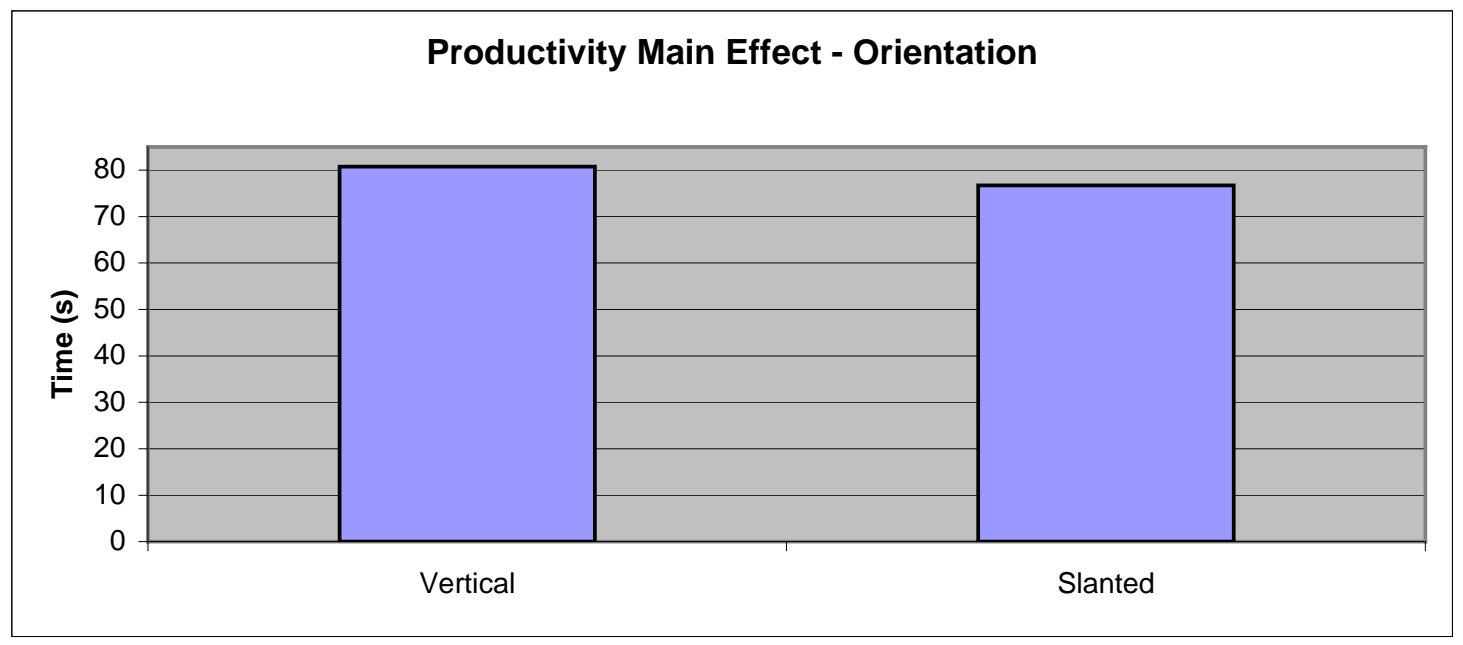

Figure 4.35: Main Effect of Orientation on Productivity. 


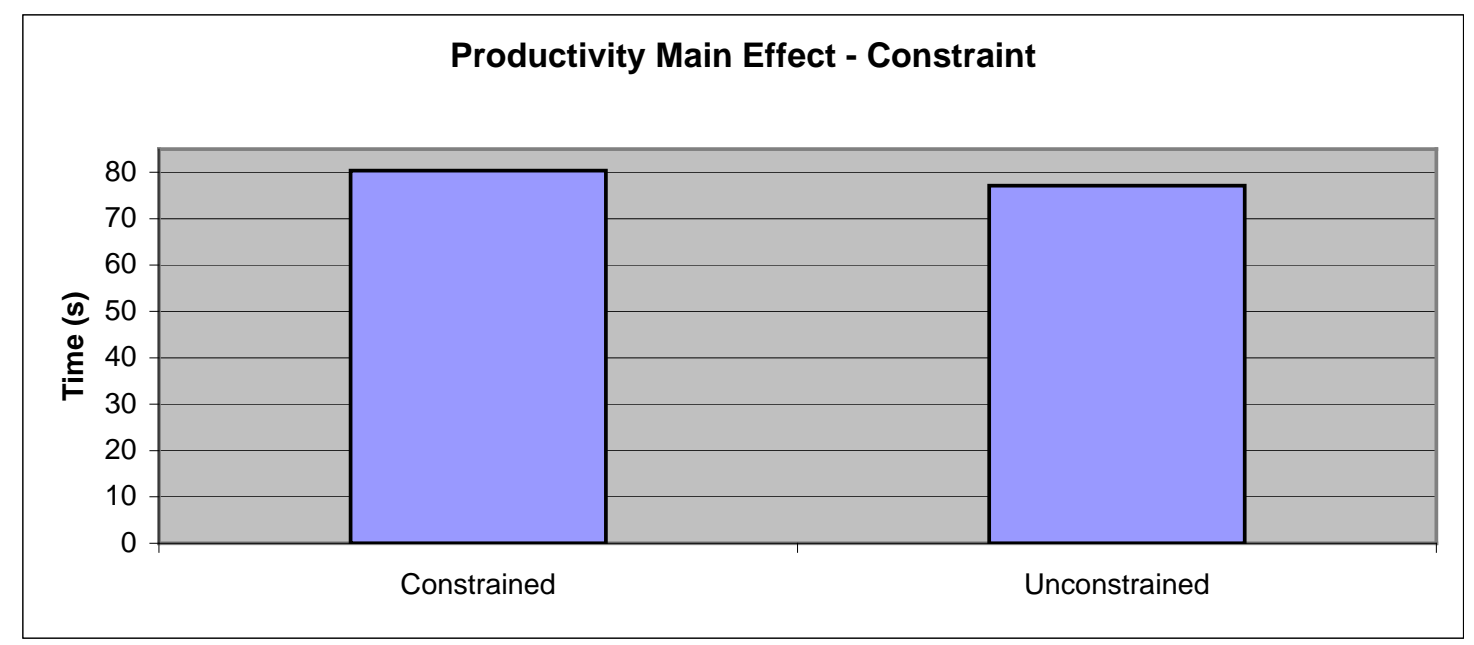

Figure 4.36: Main Effect of Constraint on Productivity.

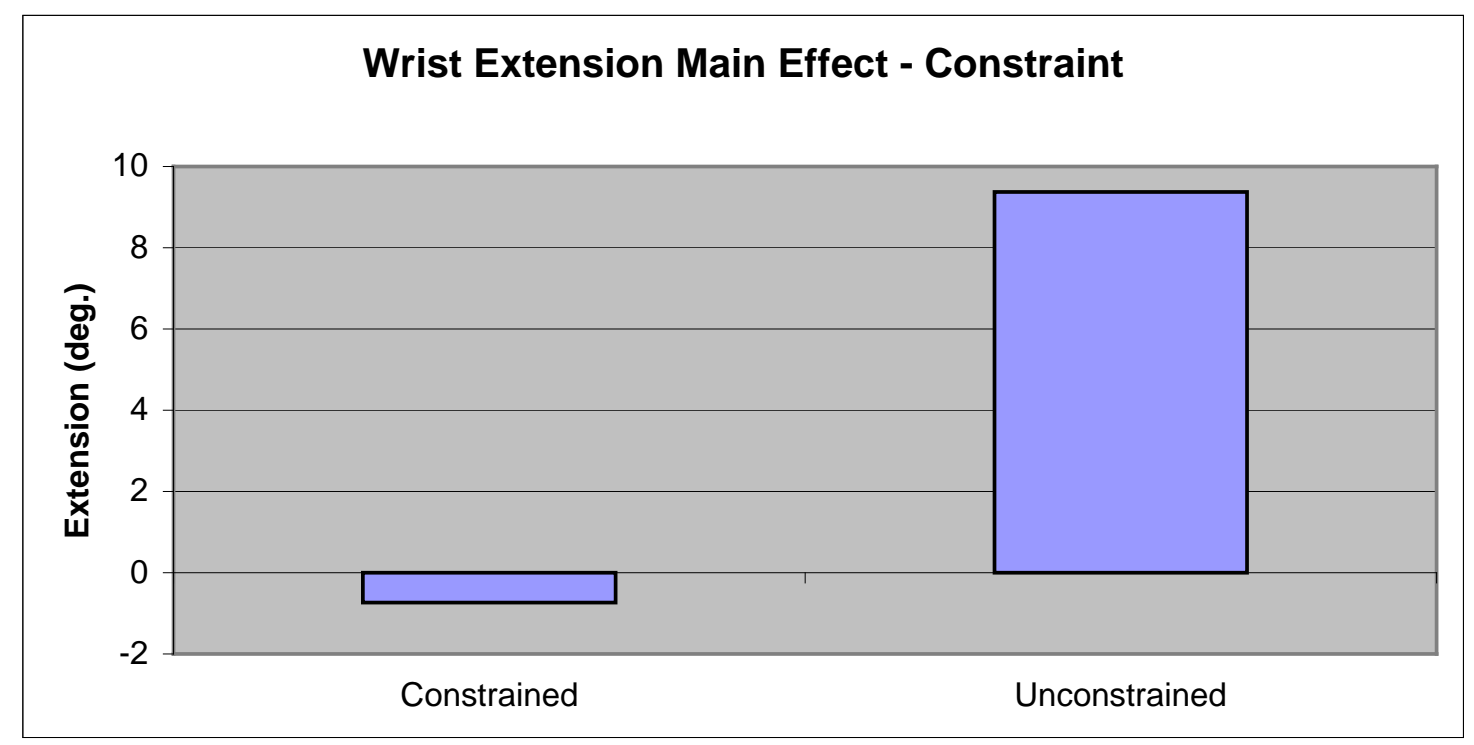

Figure 4.37: Main Effect of Constraint on Wrist Extension. 


\subsubsection{Spring Task}

Tables 4.5 through 4.8 list the results on the ANOVA (including F test statistic and Pvalue) for the four dependent variables productivity, arm elevation, wrist

flexion/extension, and wrist radial/ulnar deviation and all interactions. (All significant results - except for Subject - are bolded.)

Table 4.5: Productivity Results.

\begin{tabular}{|l|l|l|}
\hline Effect & F Test Statistic & Prob. $>$ F \\
\hline Subject & 18.62 & P $<0.0001$ \\
\hline Pliers & $\mathbf{6 . 9 7}$ & $\mathbf{P}<\mathbf{0 . 0 1}$ \\
\hline Orientation & $\mathbf{1 6 . 6 4}$ & $\mathbf{P}<\mathbf{0 . 0 0 0 1}$ \\
\hline Constraint & $\mathbf{3 . 9 6}$ & $\mathbf{P}<\mathbf{0 . 0 5}$ \\
\hline Pliers x Orientation & $\mathbf{4 . 5 9}$ & $\mathbf{P}<\mathbf{0 . 0 4}$ \\
\hline Pliers x Constraint & 2.24 & not significant \\
\hline Orientation x Constraint & 0.34 & not significant \\
\hline Pliers x Orientation x Constraint & 0.58 & not significant \\
\hline
\end{tabular}

Table 4.6: Arm Elevation Results.

\begin{tabular}{|l|l|l|}
\hline Effect & F Test Statistic & Prob. > F \\
\hline Subject & 9.81 & P $<0.0001$ \\
\hline Pliers & 3.71 & P $<0.0567$ \\
\hline Orientation & $\mathbf{1 0 . 9 8}$ & P $<\mathbf{0 . 0 0 2}$ \\
\hline Constraint & 0.07 & not significant \\
\hline Pliers x Orientation & 0.67 & not significant \\
\hline Pliers x Constraint & 0.67 & not significant \\
\hline Orientation x Constraint & 0.29 & not significant \\
\hline Pliers x Orientation x Constraint & 2.14 & not significant \\
\hline
\end{tabular}


Table 4.7: Wrist Flexion/Extension Results.

\begin{tabular}{|l|l|l|}
\hline Effect & F Test Statistic & Prob. $>$ F \\
\hline Subject & 22.46 & P $<0.0001$ \\
\hline Pliers & 1.19 & not significant \\
\hline Orientation & $\mathbf{4 1 . 2 0}$ & P $<\mathbf{0 . 0 0 0 1}$ \\
\hline Constraint & 0.71 & not significant \\
\hline Pliers x Orientation & 1.58 & not significant \\
\hline Pliers x Constraint & 0.71 & not significant \\
\hline Orientation x Constraint & 0.01 & not significant \\
\hline Pliers x Orientation x Constraint & 2.18 & not significant \\
\hline
\end{tabular}

Table 4.8: Wrist Radial/Ulnar Deviation Results.

\begin{tabular}{|l|l|l|}
\hline Effect & F Test Statistic & Prob. $>$ F \\
\hline Subject & 3.97 & P $<0.0001$ \\
\hline Pliers & $\mathbf{7 . 6 8}$ & $\mathbf{P}<\mathbf{0 . 0 0 7}$ \\
\hline Orientation & $\mathbf{6 . 4 5}$ & $\mathbf{P}<\mathbf{0 . 0 2}$ \\
\hline Constraint & 1.52 & not significant \\
\hline Pliers x Orientation & 1.13 & not significant \\
\hline Pliers x Constraint & 1.00 & not significant \\
\hline Orientation x Constraint & $\mathbf{4 . 1 4}$ & $\mathbf{P}<\mathbf{0 . 0 5}$ \\
\hline Pliers x Orientation x Constraint & 1.18 & not significant \\
\hline
\end{tabular}


Figures 4.38 and 4.39 show the significant two-way interaction effects for each of the dependent variables (spring task).

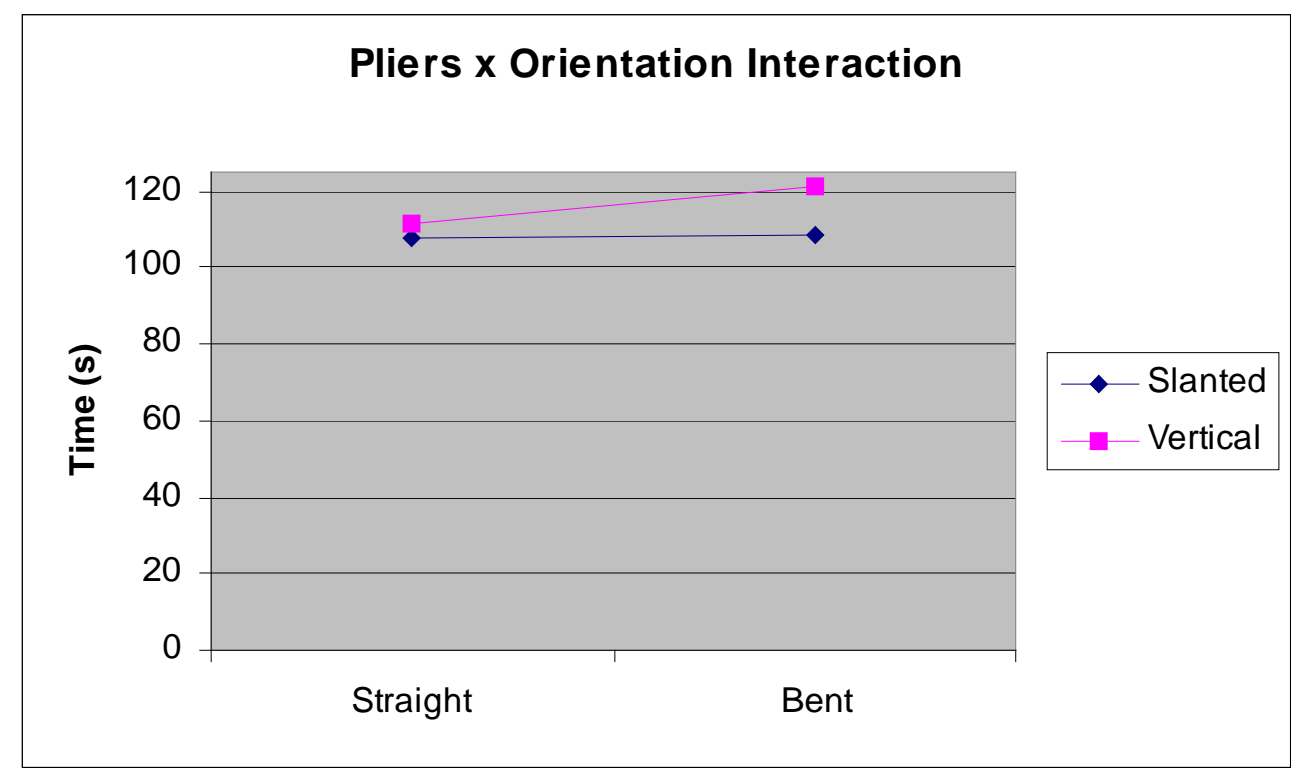

Figure 4.38: Interaction Effect of Pliers x Orientation on Productivity.

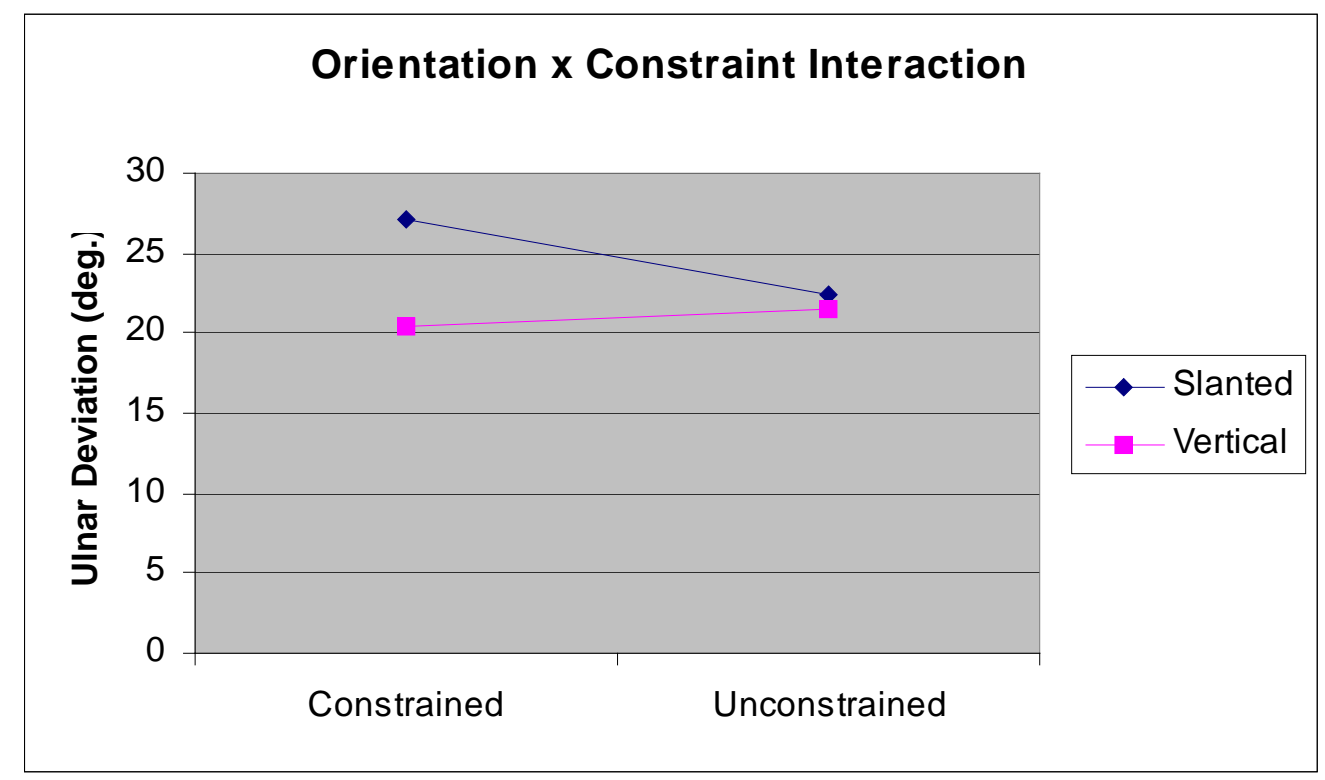

Figure 4.39: Interaction Effect of Orientation x Constraint on Ulnar Deviation. 
Figures 4.40 through 4.45 show the significant main effects for each of the dependent variables (spring task). Note: some of the significant main effects are not plotted because a significant higher order interaction invalidated the interpretation of the main effect.

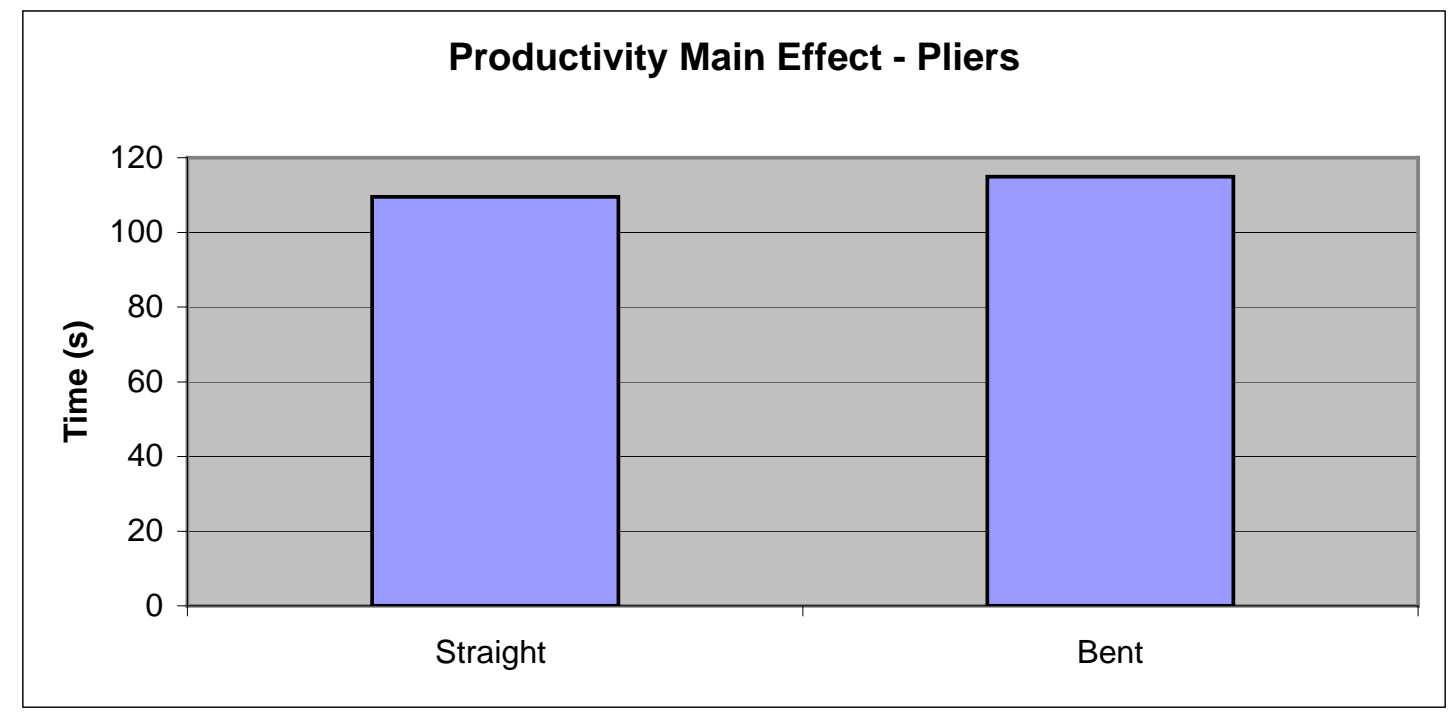

Figure 4.40: Main Effect of Pliers on Productivity.

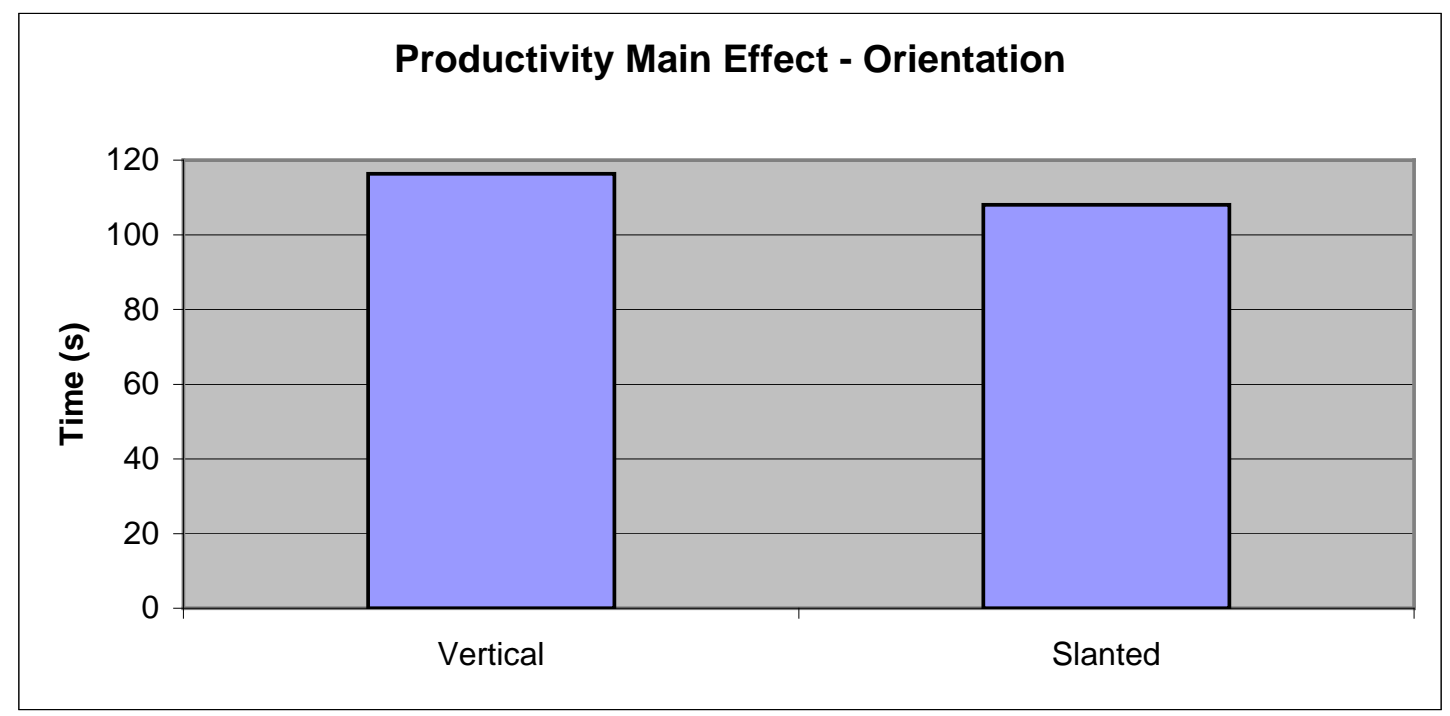

Figure 4.41: Main Effect of Orientation on Productivity. 


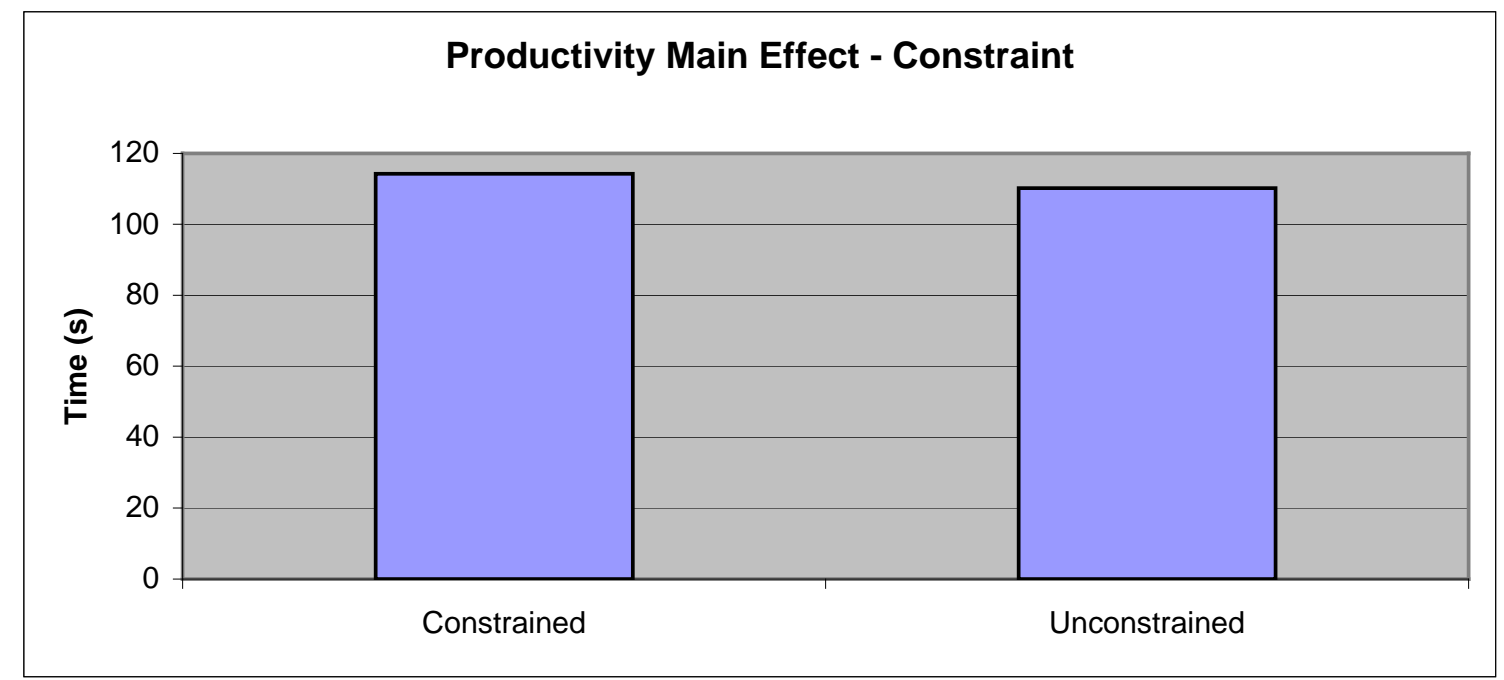

Figure 4.42: Main Effect of Constraint on Productivity.

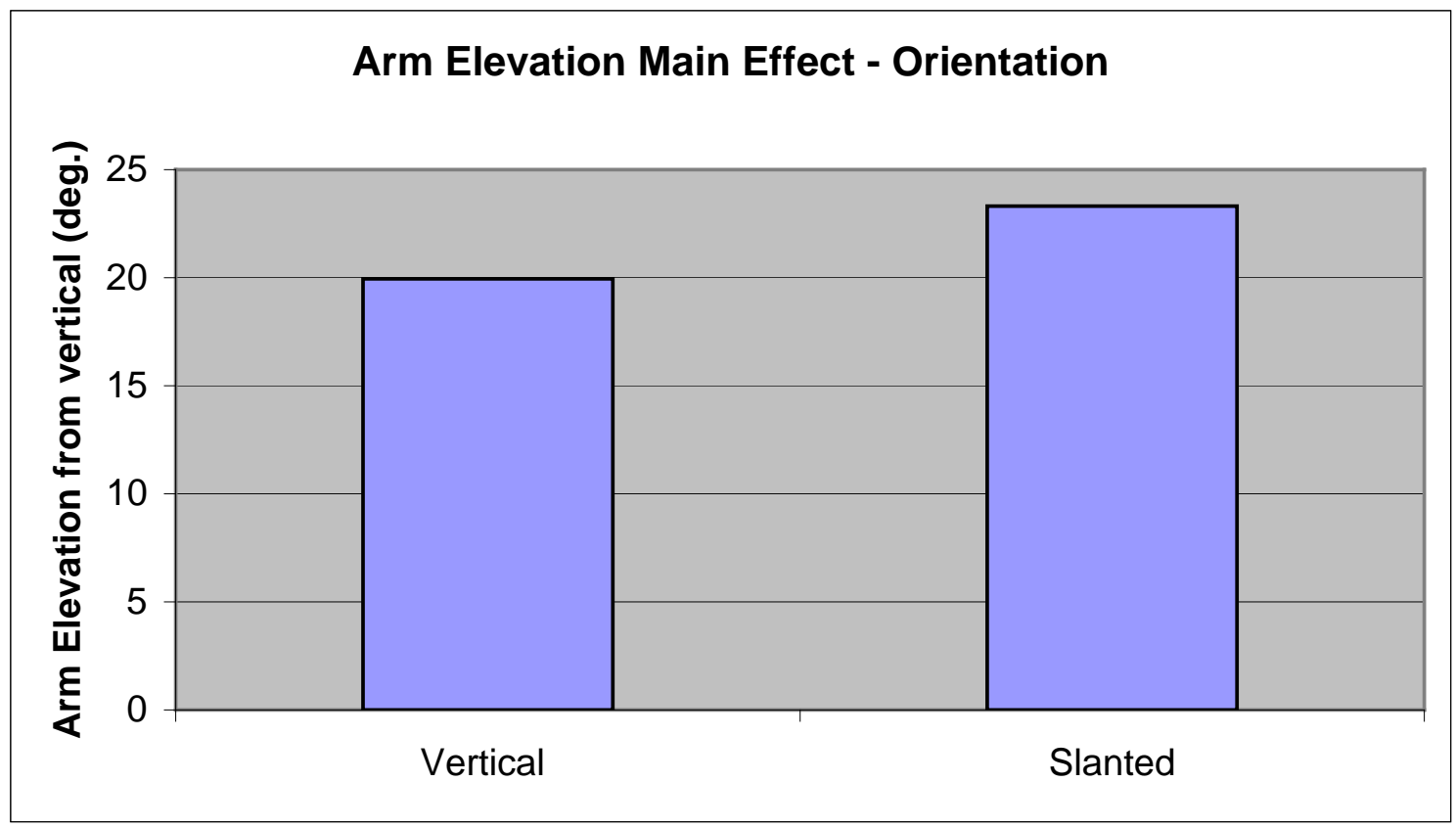

Figure 4.43: Main Effect of Orientation on Arm Elevation. 


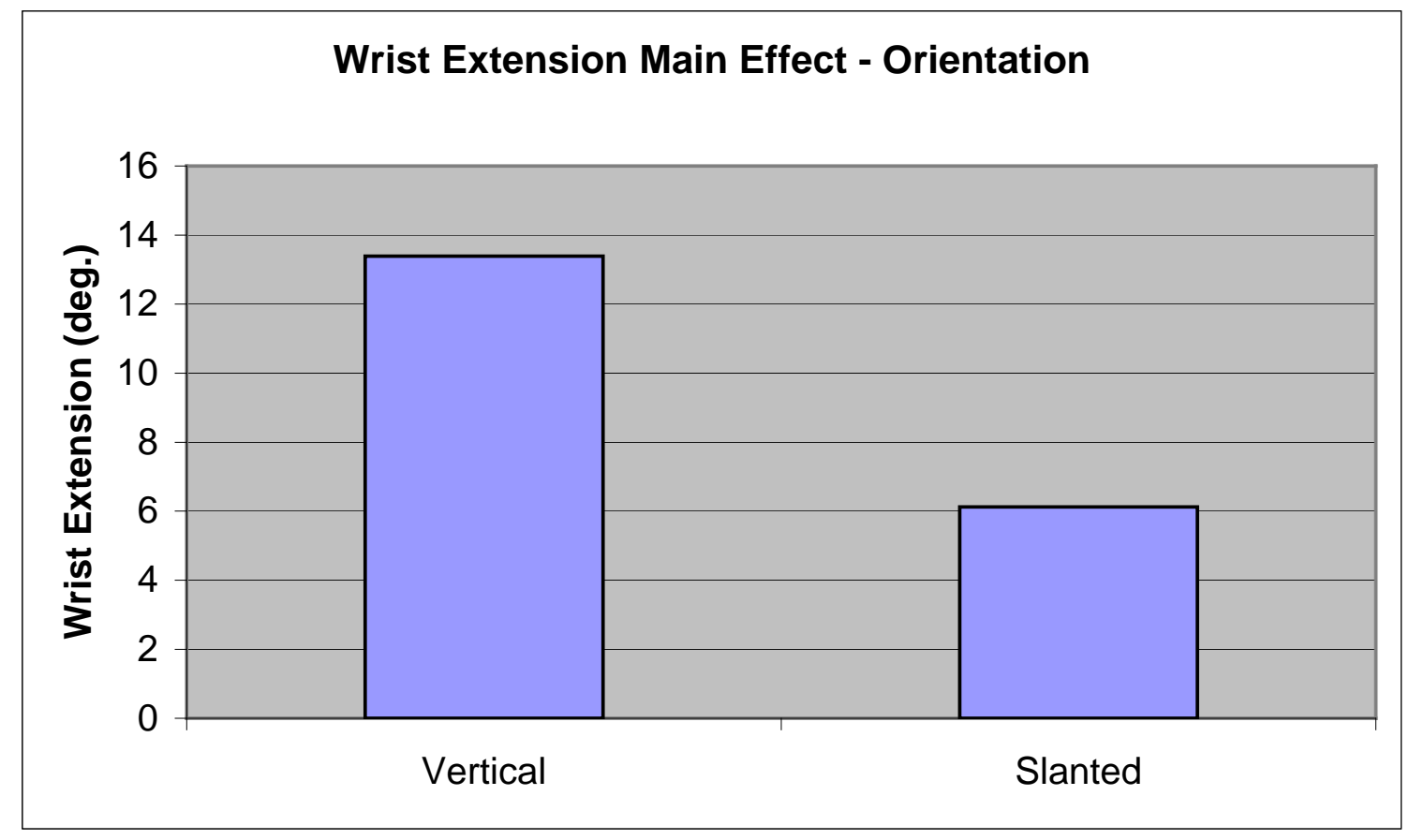

Figure 4.44: Main Effect of Orientation on Wrist Extension.

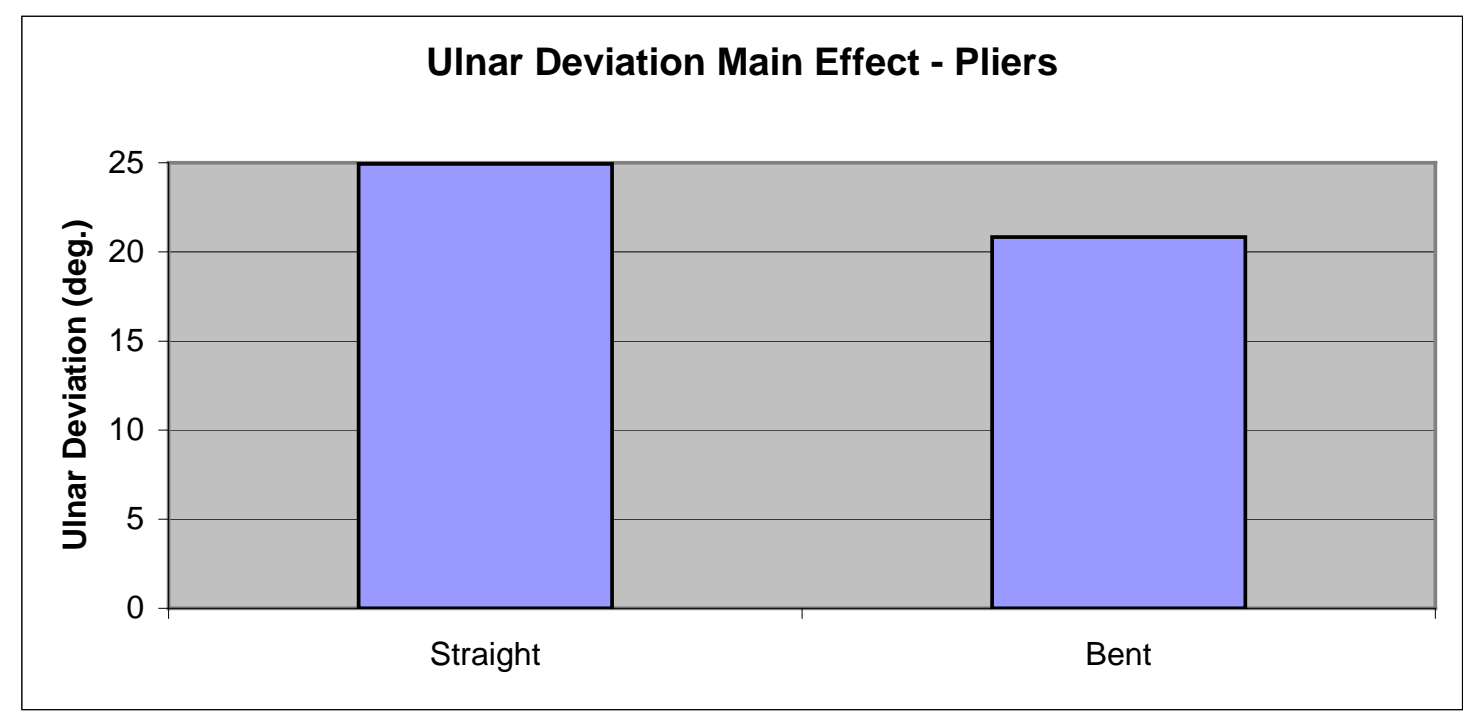

Figure 4.45: Main Effect of Pliers on Radial/Ulnar Deviation. 


\subsection{DISCUSSION}

\subsection{Results Directly Addressing Hypotheses}

Hypothesis \#1: there would be a significant productivity disadvantage with the benthandle pliers due to the constraint of the tool design (lack of symmetry about the long axis).

Both tasks had significant main effects of pliers. The results for the spring task demonstrated a productivity decrement, with the bent-handle pliers taking on average 4.9\% longer to complete the task; this supports hypothesis \#1.

On the other hand, the bent-handle pliers resulted in 5.3\% faster task performance on the jumper task; this refutes hypothesis \#1. It could be that because the jumper task requires no rotation of the pliers, the lack of long-axis symmetry has no detrimental effect on productivity, and in fact there appears to be a productivity advantage to the bent-handle pliers in this case. A likely explanation for this advantage is that there is less visual obstruction of the important task characteristics (the interaction between the pliers jaws and the jumper, and the view of the connector pins) with the bent-handle pliers compared to the straight-handle pliers. Another suggestion is that with the jumper task there were generally postural benefits of the bent-handle pliers (outlined below), and the more awkward postures required of the straight-handle pliers could have a fatiguing effect that reduces productivity.

Therefore as a general rule hypothesis \#1 is shown to be incorrect, but instead it appears that the productivity effect of the bent-handle pliers is task-specific; that is, a task that requires greater manipulation of the tool will have productivity detriments with the bent-handle pliers, but there is the potential for productivity advantages with these pliers if the task requirements resemble those described above. 
Hypothesis \#2: When held with a power grip or oblique grip, postural benefits (less radial/ulnar deviation and less arm elevation) of the bent-handle pliers are task-specific. In particular, it was expected that a task that can be performed with the pliers remaining in the sagittal plane and not requiring rotation out of this plane would have significantly less deviations with the bent-handle pliers. Conversely, tasks requiring manipulation out of this plane will favour the straight-handle pliers.

There was a significant main effect of pliers on arm elevation for the jumper task. The data for the constrained trials alone revealed that with the bent-handle pliers the average arm elevation was $19^{\circ}$, compared to $38^{\circ}$ for the straight-handle pliers; a reduction in arm elevation of 50\%. This result supports hypothesis \#2.

There was a two-way interaction effect of pliers $x$ constraint on ulnar deviation for the jumper task. Analysis of the constrained data alone revealed that ulnar deviation was $30^{\circ}$ with the bent-handle pliers, versus $34^{\circ}$ with the straight-handle pliers; this represents a reduction of $12 \%$. This result also supports hypothesis \#2.

There was a main effect of pliers on ulnar deviation for the spring task. Analysis of the data (for the constrained trials only) revealed that the mean ulnar deviation with the bent-handle pliers was $21^{\circ}$, while for the straight-handle pliers it was $27^{\circ}$ (a $22 \%$ reduction). This result refutes hypothesis \#2. There was no significant effect of pliers on shoulder elevation for the spring task; this lack of significance also refutes hypothesis \#2.

Overall hypothesis \#2 is shown to be incorrect. The results for the jumper task do support the hypothesis suggesting that the bent-handle pliers are better at reducing awkward postures for this task (and presumably for tasks with similar requirements). The results on the spring task indicate that there is still a postural advantage to the benthandle pliers (when the coupling is constrained to the grip implied by the design) for a task that requires greater degrees of end-point manipulation. 
A comparison of the significant effect on arm elevation of pliers between the two tasks shows a 50\% reduction for the jumper task compared to a non-significant result for the spring task (where the overall mean was $21^{\circ}$ ). This difference does support the general theme of this hypothesis that the effects are task-specific, however it further suggests that the difference in task requirements substantially influences the effect of the tool design, and that, at least in this case and likely most, the ability to make generalizations is very limited.

Hypothesis \#3: The specificity of the design of the bent-handle pliers would result in significant postural benefits (less radial/ulnar deviation and less arm elevation) over the straight-handle pliers when the pliers are held (constrained) to a power grip or oblique grip. Conversely, when subjects are able to hold the pliers unconstrained the postural advantage would be lost.

There was an interaction effect of pliers $x$ constraint on arm elevation for the jumper task. The interaction effect was analyzed further to show that in the constrained condition the bent-handle pliers had significantly less arm elevation than the straighthandle pliers, while in the unconstrained condition there was no significant effect of pliers. These results support hypothesis \#3.

There was an interaction effect of pliers $x$ constraint on radial/ulnar deviation for the jumper task. These results demonstrate that in the constrained condition the benthandle pliers resulted in less ulnar deviation, while in the unconstrained condition, the straight-handle pliers were better in reducing ulnar deviation. These results also support hypothesis \#3.

In the case of the spring task there were not significant effects for pliers $x$ constraint on either of the postural measures of interest; these results refute hypothesis \#3. 
Therefore, while as a general rule hypothesis \#3 is shown to be incorrect, the inference is that the effect of the constrained use of the pliers is again highly taskspecific.

\subsection{Other Significant Results}

\subsubsection{Flexion/Extension}

Flexion/extension data were collected, but there were no hypotheses made regarding the expected results since the design of the bent-handle pliers was not meant to address this posture. There was one significant main effect of orientation on flexion/extension angle for the spring task. There was one main effect of constraint on flexion/extension angle for the jumper task, and all two-way interactions were significant for the jumper task. Even though there were significant effects, the largest mean deviation in extension was approximately $11^{\circ}$, while for flexion it was approximately $5^{\circ}$. Of all the literature reviewed for this study, there were none that suggested that deviation of this degree would present any risk for development of a MSD, and so no further discussion is warranted.

\subsubsection{Productivity}

In both tasks in this study there was a significant main effect of work surface orientation on productivity, and in both cases the work surface slanted at $45^{\circ}$ resulted in faster completion times (5.1\% faster for the jumper task and $7.2 \%$ for the spring task). Although not of main interest in this study, these results suggest that with regards to productivity the slanted work surface is better. A likely explanation for this result is that the slanted work surface allows for better visual perception (particularly by eliminating parallax) for task performance. 
There was also a main effect of constraint for both tasks. None of the hypotheses addressed constraint as a main effect, but these results are of interest and will be discussed further in Section 5.3.

For the spring task there was a pliers $x$ orientation interaction, which showed that the bent-handle pliers incurred a greater productivity decrement when changing from the slanted to vertical work surface $(11.7 \%$ more time to complete the task with the benthandle pliers versus $3.7 \%$ longer with the straight-handle pliers). These results cannot be explained without further investigation.

\subsubsection{Arm Elevation (Shoulder Posture)}

For both tasks there was a main effect of orientation on arm elevation, and in both cases the slanted surface resulted in increased arm elevation; in the jumper task the arm elevation was $26 \%$ greater, while in the spring task it was $16.8 \%$ higher. These results are not surprising since as the work surface rotates away from the subject (on an axis perpendicular to the sagittal plane), the common kinematic response is to either elevate the arm and/or deviate the wrist to the ulnar side to allow the jaws of the pliers to be oriented approximately perpendicular to the work surface.

There was a pliers $x$ orientation interaction for the jumper task. This demonstrated the same effect of increased arm elevation in moving from vertical to slanted for both pliers, but the increase in arm elevation was greater for the straighthandle pliers. These results (and others) will be discussed further in Section 5.3. There was a main effect of constraint, as well as a significant interaction of constraint $x$ orientation on arm elevation for the jumper task; these results will also be addressed in Section 5.3. 


\subsubsection{Radial/Ulnar Deviation}

For the spring task there was a significant two-way interaction of orientation $x$ constraint; these results suggest that in this task unconstraining the tool coupling is only effective in changing radial/ulnar posture on the slanted work surface. This explanation can be validated by the observation that on the slanted surface subjects did change handtool coupling 16 of 32 trials, while on the vertical surface a change was made only nine of 32 trials. (These observations of the frequency of hand-coupling change during the unconstrained trials are described in more detail in section 5.3, where they are used to further explain many of the results in this experiment.)

For the jumper task there were significant effects on radial/ulnar deviation due to constraint (main), pliers $x$ orientation interaction, and orientation $x$ constraint interaction; all of these results are explained further in Section 5.3

\subsection{Further Discussion Regarding Tool Coupling}

There was an observation made during data collection that, though not validated statistically, is believed (in the opinion of the investigator) to go a long way in explaining many of the results obtained, and ultimately supports the notion that the design of the bent-handle pliers constrains users.

The design of the study had subjects perform all conditions in a randomized order of presentation of pliers by orientation; within this randomization, however, subjects always performed the constrained condition first, followed immediately by the unconstrained. (This order reflects what would likely happen in a real-world application, where a worker would start using a tool as it was designed to be used, but given the

opportunity, would explore other ways to use the tool.) Though subjects did not have to 
change coupling for the unconstrained conditions, notes taken during the experiment indicated that with the straight-handle pliers subjects did change their coupling with the tool 42 out of 64 times, while with the bent-handle pliers this was done only 18 out of 64 times (for all trials subjects were informed to perform as quickly as possible, so when choosing the coupling used during the unconstrained conditions this remained the objective).

Often the coupling change was minor, for example moving the pliers out from the palm onto the fingertips. However, there were occasions when subjects made "major" changes to the coupling (major is defined here as a departure from holding the pliers in the sagittal plane with the thumb opposed by at least three digits, and the jaws exiting from the radial aspect of the hand), and this was more frequently done with the straighthandle pliers ( 15 of 64 trials) then with the bent-handle pliers ( 6 out of 64 trials). Of these, there was a particularly common "major" coupling change that subjects made: turning the pliers so that the jaws exited from the ulnar aspect of the hand (called here, a "dagger grip"). With the bent-handle pliers subjects tried the dagger grip six out of 64 trials, while with the straight-handle pliers it was attempted 13 of 64 trials. Furthermore, of the six trials used with the bent-handle pliers only two resulted in a subjective preference for the dagger grip over the constrained grip, while with the straight-handle pliers the ratio was ten of 13. (Note: the other two major changes with the straighthandle pliers included one trial where the subject held the pliers in an extremely supinated manner that allowed the shoulder and wrist to be maintained in neutral positions, and one resulted in the pliers being held such that they exited the hand from 
between the third and fourth digits.) Tables 5.1 and 5.2. provide a summary of the coupling changes made during the unconstrained conditions.

Table 5.1: Breakdown of alternative couplings during unconstrained conditions (data from both tasks combined).

\begin{tabular}{|l|c|c|c|c|}
\hline Tool & Minor Coupling Change & Dagger Grip & $\begin{array}{c}\text { Other Major Coupling } \\
\text { Change }\end{array}$ & Total out of 64 \\
\hline Straight-handle & 27 & 13 & 2 & 42 \\
\hline Bent-handle & 12 & 6 & 0 & 18 \\
\hline
\end{tabular}

The alternative coupling numbers can be broken down further by work surface orientation as demonstrated in Table 5.2.

Table5. 2: Number of trials for which a different coupling was used during the unconstrained condition on the two orientations (data from both tasks combined).

\begin{tabular}{|l|c|c|c|}
\hline Tool & Vertical & Slanted & Total \\
\hline Straight-handle & 18 & 24 & 42 \\
\hline Bent-handle & 5 & 13 & 18 \\
\hline Total & 23 & 37 & \\
\hline
\end{tabular}

Table 5.2 demonstrates, that not only were subjects more likely to use the straight-handle pliers with different couplings, but that it was more common to change the coupling on the slanted work surface.

Relating this back to the significant results, it can be seen that for both tasks, orientation had a significant main effect; with the slanted surface the jumper task was performed 5\% faster, while the spring task was performed $7.1 \%$ faster (this was explained as likely being due, at least in part, to better visual input). Also, both tasks had a main effect of constraint on productivity, with the unconstrained conditions resulting in quicker task performance. Taking these main effects into consideration, the suggestion 
from a productivity perspective is that the slanted work surface is superior for productivity, as is the unconstrained condition.

The significant main and interaction effects on radial/ulnar deviation support this design recommendation from an ergonomic perspective. The pliers $x$ constraint interaction (jumper task) demonstrated that deviation was minimized with the unconstrained, straight-handle pliers (47.3\% less than the bent-handle pliers in the unconstrained condition). While for the orientation $x$ constraint interaction (jumper task) deviation was minimized for the unconstrained condition on the slanted work surface (the slanted unconstrained condition was $32.2 \%$ less than the vertical unconstrained). The only interaction effect on posture for the spring task was orientation $x$ constraint on radial/ulnar deviation, but these results do not support this argument.

Similarly, looking at the significant effects on shoulder posture, it can be seen that for the jumper task there was a significant main effect of constraint and that this effect showed reduced shoulder deviation (28.3\% less) when the pliers are used unconstrained. Additionally there was an orientation $x$ constraint interaction effect that showed both orientations are positively impacted by the unconstrained condition, and that the improvement is greater for the slanted orientation (the slanted orientation reduced arm elevation by $39 \%$, while the vertical orientation reduced it by only $12.6 \%$ ).

The consistent theme of these results is that when tool-users hold the pliers unconstrained they are able to maintain more neutral postures, and that this benefit is more pronounced with the straight-handle pliers. Furthermore, it supports performance of the task on a slanted surface, where performance may be quicker. 


\subsection{Relating This Study to Previous Works}

The previous investigations into bent-handle designs for hammers, files, and knives (cited earlier in this document) all found favourable results, in one form or another, of the bent-handle design over the conventional, straight-handle counterpart. However, it is difficult to relate the results when the outcomes of interest are not the same.

This study investigated both the ergonomic impact (via posture) and the productivity impact of the two different designs. Schoenmarklin and Marras (1989a) found postural differences between the different hammer designs in the maximum amount of ulnar deviation, but not in the total range of motion, while they did not find productivity differences. Similarly, Hsu and Chen (1999) found postural benefits to their bent-handle file design, but there were no differences in tests of precision or efficiency (except for the extreme $90^{\circ}$ bend, which was worse than all others, including the straighthandle design). The investigation into bent-handle knives did not explore productivity, and lastly, there is disagreement between the productivity results of Tichauer (1968) and Dempsey and Leamon (1995). This study found a productivity advantage to the benthandle pliers for one task, and a decrement in the other task. It is important to consider the motivational role of productivity, if it is supported or opposed by postural benefits, and if the two conflict, how much does each contribute to the decision regarding which tool to use. These are all factors that are not only task specific, but certainly weigh differently amongst individuals.

Many of the earlier studies relied on subjective evaluation of the two different designs for hand tools. Granada and Konz (1981), Krohn and Konz (1982), and Konz 
and Street (1984), all used college students as subjects to evaluate the tool designs. Only Hsu and Chen (1999) used experienced subjects, and though they did find subjective preferences with the bent-handle design, the results of one study cannot be applied to industry in general. Would we find consistent results if experienced subjects were used in all the studies? It is worth reminding the reader that in their discussion section, Hsu and Chen (1999) noted that workers should have both a bent-handle file and a conventional straight-handle tool at their disposal.

Another consideration is that, as with this investigation, all but one of the works cited here were laboratory studies; the only previous work found in the ergonomics literature that utilized a field study to asses the effects of a bent-handle design was that of Tichauer (1973), and a review by Leamon and Dempsey (1995) indicates "the validity of Tichauer's results needs further support". Overall, the lack of field results for this design issue has major implications on the face validity of the results, and the subsequent recommendation that has been emphasized as a hand-tool design tenet, must be reevaluated.

An article by Dempsey and Leamon (1995) provides further rationale for understanding (and perhaps underplaying) the ergonomic impact that bent-handle tools may have. In their article they outline a model that shows tool design being just one of four major factors (the others being workplace design, operator characteristics, and task characteristics), with handle shape being just one of five tool design characteristics, that ultimately determine wrist deviation. Their model shows tool design interacting with operator characteristics, and as has been clearly emphasized in this paper, it is likely that for real world uses of the pliers (and other tools designed to this tenet) the bent-handle 
design is likely to constrain the operator. Again the reader is reminded that both Schoenmarklin and Marras ((1989a), and Konz and Streets (1984) alluded to this in the respective discussion sections of their bent-handle hammer studies; stating that the changes in the design of the tool handle cannot be done independently of the task, and that the bend in the handle can constrain the user's grip.

\subsection{Future Research}

The motivation for this study was the lack of congruence between the common ergonomic recommendation to "bend the tool" (specifically the example of the pistol-grip pliers) and the lack of industry use of this tool. One of the primary hypotheses was that the lack of symmetry about the long axis of the bent-handle pliers constrained the user of the tool. Further studies should be developed to investigate the relationship between tool design symmetry and user constraints.

Additionally, it was hypothesized that the results were task specific, and that tasks requiring manipulation of the jaws of the pliers outside of the sagittal plane were less suited to use of the bent-handle pliers (as determined by the dependent variables in this study). It would be worthwhile evaluating a task that was identical in all respects (for example: task goal, interaction between part and pliers, equal travel distances, equal visual conditions) but that had as an independent variable tool manipulation in different planes.

Ultimately, what is needed is an example from industry of a task that currently uses the bent-handle pliers (or any of the other bent-handle tools mentioned) that could be used for a field study to analyze the productivity and ergonomic implications of this design recommendation. 


\subsection{Limitations}

The postural data collected in this study were averaged to produce one value per subject per trial. Although pilot data indicated that the range of motion in any of the dependent variables measured was relatively small, there may have been additional information contained in this data that could provide insight into the implications of the bent-handle pliers design.

During the unconstrained trials subjects were not permitted to change their coupling once they started the trial. This was done to reduce the complexity of the study, and to reduce the variability, however it does not reflect the real world where tool users would likely be able to change their coupling (and other task factors) to shift the strain of the task during performance.

A detailed description of the work surface height determination was provided for this study. It is believed that the approach taken for determining this task characteristic was appropriate, however the work surface height has a great deal of influence on the dependent variables and the effect on the study results cannot be known. Expanding the study to include an independent variable of height would potentially provide insight, but would also dramatically increase the scope of the study.

As mentioned in the Introduction, there were no industry tasks found where the bent-handle pliers were being used. This would have provided a superior method for analyzing the effects of the bent-handle pliers compared to the straight-handle pliers. Although it is believed that the fabricated tasks were good representatives of real world tasks, this may not have been the case. 
Subjects used in this study had varying degrees of experience with pliers; this likely introduced more variability into the study, making the identification of significant results more difficult. Similarly, experienced workers (with a real task) may have a different strategy for using the pliers that the recruited subjects in this study would not have necessarily used.

The short duration of the trials may have altered the results, since longer use may require different strategies. For example, subjects may have been able to provide higher productivity over the shorter period of time, and they may have been able to maintain awkward postures for the durations used in this study; not reflecting what may occur in a real work environment. 


\subsection{CONCLUSION}

Although the results of this study did not fully corroborate the original hypotheses, there were many significant results that did support them to varying degrees. Furthermore, a line of logic was developed based indirectly on the results that suggested that the design of the bent-handle pliers constrains users for the tasks used in this study, and that the constraint of the design has the potential to reduce productivity and cause more awkward wrist and shoulder postures.

There is the suggestion that the bent-handle pliers would be better for a task that has very specific characteristics (sagittal plane orientation with no rotation out of this plane), but the difficulty in finding an industry example with these characteristics leaves this question unanswered. Overall, the results suggest that a hand tool designed for specificity can impose constraints on the user, and in such a case a flexible design is better.

A final conclusion to be made from these results, and those of previous works, is that this design recommendation cannot be made in the absence of a clear and complete understanding of the task requirements, the user population, and the environmental conditions. 


\section{REFERENCES}

Armstrong, T.J., \& Chaffin, D.B. (1979). Some Biomechanical Aspects of the Carpal Tunnel. Journal of Biomechanics, 12(7), 567-570.

Bridger, R.S. (1995). Introduction to Ergonomics. New York: McGraw-Hill Inc.

Bureau of Labor Statistics, United States Department of Labor (2000, April 10). Lost-worktime injuries and illnesses: characteristics and resulting time away from work. News Release retrieved May 21, 2002 from the World Wide Web:'inttp://stats.bis. gov/iif/home.htm

Carey EJ, Gallwey TJ. The Relationship of Wrist Posture to Discomfort during Repetitive Exertions. In: Contemporary Ergonomics 2000. McCabe PT, Hanson MA, Robertson SA (Eds.). London: Taylor \& Francis; 2000; 286-290.

Chaffin, D.B., Andersson, G.B.J., \& Martin, B.J. (1999). Occupational Biomechanics ( $3^{\text {rd }}$ Ed.). New York: John Wiley and Sons Inc.

Dempsey, P.G., \& Leamon, T.B. (1995). Bending the tool and the effect on productivity - An investigation of a simulated wire-twisting task. American Industrial Hygiene Association Journal, 56(7), $686-692$.

Dempsey, P.G., \& Leamon, T.B. (1995). Implementing bent-handle tools in the workplace. Ergonomics in Design, 1995; October: 15-21.

Fogleman, M.T., Freivalds, A., \& Goldberg, J.H. (1993). An ergonomic evaluation of knives for two poultry cutting tasks. International Journal of Industrial Ergonomics, 11, 257-265.

Granada, M. \& Konz, S. (1981). Evaluation of bent hammer handles. Proceedings of the Human Factors Society, $25^{\text {th }}$ Annual Meeting, 322-324.

Herberts, P., Kadefors, R., Hogfors, \& Sigholm, G. (1984). Shoulder Pain and Heavy Manual Labour. Clinical Orthopaedic, 191, 166-178.

Hsu, SH., \& Chen, Y.H. (1999). Evaluation of bent-handle files. International Journal of Industrial Ergonomic, 25, 1-10.

Keir, P.J., Bach, J.M., Engstrom, J.W., \& Rempel, D.M. (1996). Carpal Tunnel Pressure: Effects of Wrist Flexion/Extension. Presented at the $20^{\text {th }}$ Annual Meeting of the American Society of Biomechanics, Atlanta. Retrieved May 30, 2002 from the American Society of Biomechanics website:'http://asb-biomech.org/onlineabs/abstracts $96 / \mathrm{keir}$ html

Kolarik, W.J. (1995). Creating Quality: Concepts, Systems, Strategies, and Tools. New York: McGrawHill, Inc.

Konz, S. \& Johnson, S. (2000). Work Design: Industrial Ergonomics (5 $5^{\text {th }}$ Ed.). Scotsdale AZ: Holcomb Hathaway.

Konz, S., \& Streets, B. (1984). Bent hammer handles: Performance and preference. In: Proceedings of the Human Factors Society, 28the Annual Meeting, Santa Monica, CA: The Human Factors Society, 438-440. 
Kroemer, K., Kroemer, H., \& Kroemer-Elbert, K. (1994). Ergonomics: How to Design for Ease and Efficiency. Cliffs, NJ: Prentice Hall, Englewood.

Krohn, R. \& Konz, S. (1982). Bent hammer handles. Proceedings of the Human Factors Society, 26 ${ }^{\text {th }}$ Annual Meeting, 413-417.

Leamon TB, Dempsey PG. The unusual congruence between subjective evaluations and losses associated with inadequate hand tool design. Industrial Ergonomics. 1995; 16: 23-28.

Lewis, W.G., \& Narayan, C.V. (1993). Design and Sizing of Ergonomic Handles for Hand Tools. Applied Ergonomics, 24, 351-356.

Lundborg, G., Gelberman, R.H., Minteer-Convery, M., \& Lee, Y.F. (1982). Median nerve compression in the carpal tunnel - Functional response to experimentally induced controlled pressure. Journal of Hand Surgery, 7(3), 252-259.

Marklin, R.W. (1999). Biomechanical Aspects of CTDs. In Karwowski, W. \& Marras, W.S. (eds.), The Occupational Ergonomics Handbook (pp. 795-832). New York: CRC Press.

Marras, W.S. (1999). Occupational Biomechanics. In Karwowski, W. \& Marras, W.S. (eds.), The Occupational Ergonomics Handbook (pp. 775-794). New York: CRC Press.

Marras, W.S., \& Schoenmarklin, R.W. (1993). Wrist motions in industry. Ergonomics, 36(4), 341-351.

Montgomery, D.C. (1984). Design and Analysis of Experiments (2 ${ }^{\text {nd }}$ Ed.). New York: John Wiley \& Sons.

National Institute for Occupational Safety and Health, United States Department of Health and Human Services, Public Health Service, Centers for Disease Control and Prevention. (1997).

Musculoskeletal disorders (MSDs) and workplace factors: a critical review of the epidemiological evidence for work-related musculoskeletal disorders of the neck, upper extremity, and low back (Second printing). Cincinnati OH: Author.

National Research Council and Institute of Medicine. (2001). Musculoskeletal disorders and the workplace. Panel on musculoskeletal disorders and the workplace. Commission on behavioral and social sciences and education. Washington, D. C.: National Academy Press.

Noah, D., Weiss, Gordon, L., Bloom, T., Yuen So, \& Rempel, D.M. (1995). Position of the Wrist Associated with the Lowest Carpal-Tunnel Pressure: Implications for Splint Design. Journal of Bone and Joint Surgery, 77, 1695-1699.

Occupational Safety \& Health Administration, United States Department of Labor. (1999). Preventing work-related musculoskeletal disorders. Retrieved May 22, 2002 from the World Wide Web (OSHA website):'http:///www.osha-slc.gov/S̄LTC/ergonomics/ergofactnew.html

Pulat, B.M. (1997). Fundamentals of Industrial Ergonomics. Prospect Heights, Il: Waveland Press Inc.

Rempel, D.M., Bach, J.M., Gordon. L,, \& Yuen So. (1998). Effects of Forearm Pronation/Supination on Carpal Tunnel Pressure. Journal of Hand Surgery, 23A (1), 38-42. 
Schoenmarklin, R.W., \& Marras, W.S. (1989a). Effects of Handle Angle and Work Orientation on Hammering: I. Wrist Motion and Hammering Performance. Human Factors, 31(4), 397-411.

Schoenmarklin, R.W., \& Marras, W.S. (1989b). Effects of Handle Angle and Work Orientation on Hammering: II. Muscle Fatigue and Subjective Ratings of Body Discomfort. Human Factors, 31(4), 413-420.

Tichauer, E.R. (1968). Potential of Biomechanics for Solving Specific Hazard Problems. In: Proceedings of 1968 Professional Conference. Park Ridge IL: American Society of Safety Engineers.

Tichauer, E.R. (1973). Ergonomic aspects of biomechanics. In: The Industrial Environment - Its Evaluation and Control (pp. 431-492). U.S. Department of Health, Education and Welfare, National Institute of Occupational Safety and Health, Washington D.C.

Tichauer, E.R. (1975). Occupational biomechanics and the development of work tolerance. In P.V. Komi (Ed.), Biomechanics V-A - Proceedings of the Fifth International Congress of Biomechanics, Jyvaskyla, Finland (pp. 493-505). Baltimore MD: University Park Press.

Tichauer, E.R. (1976). Biomechanics sustains occupational safety and health. Industrial Engineering, $8(2), 46-56$.

Tichauer, E.R. (1977). Ergonomics principles basic to hand tool design. American Industrial Hygiene Association Journal, 38(11), 622-634.

Viikari-Juntura, E. (1999). Occupational Risk Factors for Shoulder Disorders. In Karwowski, W. \& Marras, W.S. (eds.), The Occupational Ergonomics Handbook (pp. 833-849). New York: CRC Press.

Wells, R. (1999). Integrated Analysis of Upper Extremity Disorders. In Karwowski, W. \& Marras, W.S. (eds.), The Occupational Ergonomics Handbook (pp. 775-794). New York: CRC Press. 


\subsection{APPENDICES}

\subsection{Work Surface Height Determination Data}

Table 7.1: Pilot Subject Anthropometry Data.

\begin{tabular}{|l|c|c|c|}
\hline & Pilot Subject \#1 & Pilot Subject \#2 & Pilot Subject \#3 \\
\hline Shoulder Height & 161.4 & 132.8 & 146.5 \\
\hline Elbow Height & 122.6 & 102.6 & 115 \\
\hline Mid-arm & 142 & 117.7 & 130.8 \\
\hline
\end{tabular}

Table 7.2: Pilot Subject Preferred Work Heights and Averages for Jumper Task.

\begin{tabular}{|l|c|c|c|}
\hline Jumper Task & Pilot Subject \#1 & Pilot Subject \#2 & Pilot Subject \#3 \\
\hline Straight/Slanted & 113 & 106.7 & 111.1 \\
\hline Bent/Slanted & 120.7 & 110.5 & 119.4 \\
\hline Slanted Average & $\mathbf{1 1 6 . 9}$ & $\mathbf{1 0 8 . 6}$ & $\mathbf{1 1 5 . 3}$ \\
\hline Straight/Vertical & 137.8 & 114.6 & 125.7 \\
\hline Bent/Vertical & 150.5 & 118.1 & 135.9 \\
\hline Vertical Average & $\mathbf{1 4 4 . 2}$ & $\mathbf{1 1 7 . 5}$ & $\mathbf{1 3 0 . 8}$ \\
\hline
\end{tabular}

Table 7.3: Pilot Subject Preferred Work Heights and Averages for Spring Task.

\begin{tabular}{|l|c|c|c|}
\hline Spring Task & Pilot Subject \#1 & Pilot Subject \#2 & Pilot Subject \#3 \\
\hline Straight/Slanted & 123.5 & 115.6 & 117.5 \\
\hline Bent/Slanted & 127.6 & 113.7 & 121.9 \\
\hline Slanted Average & $\mathbf{1 2 5 . 6}$ & $\mathbf{1 1 4 . 7}$ & $\mathbf{1 1 9 . 7}$ \\
\hline Straight/Vertical & 134.6 & 117.5 & 128 \\
\hline Bent/Vertical & 151.1 & 117.5 & 132.1 \\
\hline Vertical Average & $\mathbf{1 4 2 . 9}$ & $\mathbf{1 1 7 . 5}$ & $\mathbf{1 3 0 . 1}$ \\
\hline
\end{tabular}

Table 7.4: Differences Between Task/Orientation Average Height $\left(\mathrm{H}_{\mathrm{avg}}\right.$ (data from Tables 2 \& 3)) and Three Body Landmarks (data from Table 1); for each pilot subject.

\begin{tabular}{|c|c|c|c|c|}
\hline & & Pilot Subject \#1 & Pilot Subject \#2 & Pilot Subject \#3 \\
\hline \multirow{3}{*}{ Jumper/Slanted } & Shoulder $-\mathrm{H}_{\mathrm{avg}}$ & -44.55 & -24.2 & -31.25 \\
\hline & Elbow $-\mathrm{H}_{\mathrm{avg}}$ & -5.75 & 6 & 0.25 \\
\hline & Mid-arm - $\mathrm{H}_{\mathrm{avg}}$ & -25.15 & -9.1 & -15.5 \\
\hline \multirow{3}{*}{ Jumper/Vertical } & Shoulder $-\mathrm{H}_{\mathrm{avg}}$ & -17.25 & -16.45 & -15.7 \\
\hline & Elbow $-\mathrm{H}_{\mathrm{avg}}$ & 21.55 & 13.75 & 15.8 \\
\hline & Mid-arm - $\mathrm{H}_{\mathrm{avg}}$ & 2.15 & -1.35 & 0.05 \\
\hline \multirow{3}{*}{ Spring/Slanted } & Shoulder $-\mathrm{H}_{\mathrm{avg}}$ & -35.85 & -18.15 & -26.8 \\
\hline & Elbow $-\mathrm{H}_{\mathrm{avg}}$ & 2.95 & 12.05 & 4.7 \\
\hline & Mid-arm - $\mathrm{H}_{\mathrm{avg}}$ & -16.45 & -3.05 & -11.05 \\
\hline \multirow{3}{*}{ Spring/Vertical } & Shoulder $-\mathrm{H}_{\mathrm{avg}}$ & -18.55 & -15.3 & -16.45 \\
\hline & Elbow $-\mathrm{H}_{\mathrm{avg}}$ & 20.25 & 14.9 & 15.05 \\
\hline & Mid-arm - $\mathrm{H}_{\mathrm{avg}}$ & 0.85 & -0.2 & -0.7 \\
\hline
\end{tabular}


Table 7.5: Differences between subjects (body landmark - $\mathrm{H}_{\text {avg }}$ (data from Table 4)) and rank by least difference between subjects.

\begin{tabular}{|l|l|c|c|c|c|}
\hline & & PS\#1 - PS\#2 & PS\#1 - PS\#3 & PS\#3 - PS\#2 & Rank \\
\hline \multirow{3}{*}{ Jumper/Slanted } & Shoulder $-\mathrm{H}_{\text {avg }}$ & -20.35 & -13.3 & -7.05 & 3 \\
\cline { 2 - 6 } & Elbow - $\mathrm{H}_{\text {avg }}$ & -11.75 & -6 & -5.75 & 1 \\
\cline { 2 - 6 } & Mid-arm - $\mathrm{H}_{\text {avg }}$ & -16.05 & -9.65 & -6.4 & 2 \\
\hline \multirow{3}{*}{ Jumper/Vertical } & Shoulder - $\mathrm{H}_{\text {avg }}$ & -0.8 & -1.55 & 0.75 & 1 \\
\cline { 2 - 6 } & Elbow - $\mathrm{H}_{\text {avg }}$ & 7.8 & 5.75 & 2.05 & 3 \\
\cline { 2 - 6 } & Mid-arm - $\mathrm{H}_{\text {avg }}$ & 3.5 & 2.1 & 1.4 & 2 \\
\hline \multirow{3}{*}{ Spring/Slanted } & Shoulder - $\mathrm{H}_{\text {avg }}$ & -17.7 & -9.05 & -8.65 & 3 \\
\cline { 2 - 6 } & Elbow $-\mathrm{H}_{\text {avg }}$ & -9.1 & -1.75 & -7.35 & 1 \\
\cline { 2 - 6 } & Mid-arm - $\mathrm{H}_{\text {avg }}$ & -13.4 & -5.4 & -8 & 2 \\
\hline & Shoulder $-\mathrm{H}_{\text {avg }}$ & -3.25 & -2.1 & -1.15 & 2 \\
\cline { 2 - 6 } & Elbow $-\mathrm{H}_{\text {avg }}$ & 5.35 & 5.2 & 0.15 & 3 \\
\cline { 2 - 6 } & Mid-arm - $\mathrm{H}_{\text {avg }}$ & 1.05 & 1.55 & -0.5 & 1 \\
\hline
\end{tabular}




\subsection{Sample Data Collection}

Table 7.6: Example of major steps for a subject data-collection period.

\begin{tabular}{|c|c|}
\hline Step 1 & Study description and completion of informed consent. \\
\hline Step 2 & Anthropometric data collection. \\
\hline Step 3 & Instrumentation. \\
\hline Step 4 & Neutral-wrist data collection (two files) \\
\hline Step 5 & Explanation and demonstration of spring task. \\
\hline Step 6 & $\begin{array}{l}\text { Practice spring task with bent pliers on vertical work surface, followed by practice with } \\
\text { straight pliers on vertical work surface }{ }^{1} \text {. }\end{array}$ \\
\hline Step 7 & $\begin{array}{l}\text { Practice spring task with bent pliers on slanted work surface, followed by practice with straight } \\
\text { pliers on slanted work surface }{ }^{1} \text {. }\end{array}$ \\
\hline Step 8 & $\begin{array}{l}\text { Data collection trial for spring task; sample order of performance }{ }^{2}: \\
\text { - } \quad \text { Bent pliers, vertical work surface, constrained. } \\
\text { - } \quad \text { Bent pliers, vertical work surface, unconstrained. } \\
\text { - } \text { Bent pliers, slanted work surface, constrained. } \\
\text { - } \quad \text { Straight pliers, slanted work surface, unconstrained. } \\
\text { - } \quad \text { Straight pliers, slanted worface, constrained. } \\
\text { - } \quad \text { Straight pliers, vertical work surface, consonstrained. } \\
\text { Straight pliers, vertical work surface, unconstrained. }\end{array}$ \\
\hline Step 9 & Explanation and demonstration of Jumper task. \\
\hline Step 10 & $\begin{array}{l}\text { Practice jumper task with bent pliers on vertical work surface, followed by practice with } \\
\text { straight pliers on vertical work surface }{ }^{1} \text {. }\end{array}$ \\
\hline Step 11 & $\begin{array}{l}\text { Practice jumper task with bent pliers on slanted work surface, followed by practice with } \\
\text { straight pliers on slanted work surface }{ }^{1} \text {. }\end{array}$ \\
\hline Step 12 & $\begin{array}{l}\text { Data collection trial for jumper task; sample order of performance }{ }^{2}: \\
\text { - } \quad \text { Straight pliers, slanted work surface, constrained. } \\
\text { - } \text { Straight pliers, slanted work surface, unconstrained. } \\
\text { - } \text { Bent pliers, vertical work surface, constrained. } \\
\text { - } \text { Straight pliers, vertical work surface, constrained. } \\
\text { - } \text { Straight pliers, vertical work surface, unconstrained. } \\
\text { - } \text { Bent pliers, slanted work surface, constrained. } \\
\text { Bent pliers, slanted work surface, unconstrained. }\end{array}$ \\
\hline Step 13 & Final neutral-wrist data collection. \\
\hline Step 14 & noval of instrumentation and end of subject data collection. \\
\hline
\end{tabular}

1. Order of performance was alternated between subjects.

2. Order of performance of pliers by orientation was randomized among subjects, but constrained condition always immediately preceded unconstrained condition for all combinations of pliers by orientation. 


\subsection{Informed Consent Form}

\section{North Carolina State University INFORMED CONSENT FORM}

Productivity and Ergonomic Investigation of Bent-Handle Pliers.

Principal Investigator: Kelly Duke

Faculty Sponsor: Gary Mirka

You are invited to participate in a research study. The purpose of this study is to determine if there are benefits to using benthandled needle-nose pliers versus straight pliers in certain tasks.

\section{INFORMATION}

In preparation for the experiment motion sensors will be placed on your dominant arm and secured using hypoallergenic tape. You will then be asked to move your hands through their full range of motion for calibration purposes. During this experiment you will be asked to perform two different tasks as quickly as possible. The first task will require you to use two different kinds of pliers to move computer jumpers between mounting pins on a board. The second task will require you to use two different kinds of pliers to stretch a small spring and attach it to a post on the work surface. Both of the tasks will be performed on work surfaces in two different orientations (vertical and $45^{\circ}$ ) with both the straight pliers and the bent-handle pliers. The entire session will be videotaped.

This study will occur in one session and will require approximately 90 total minutes of your time.

\section{RISKS}

Your participation in this study involves minimal risk. This experiment will require the use of the upper extremity and may cause discomfort in those individuals with chronic or acute problems of the dominant side arm. If you have a history of chronic problems of the shoulder, arm, elbow, forearm or hand wrist please tell the researchers. If you do not have such problems, please mark your initials here: ___ . There is some risk of skin irritation to people with very sensitive skin, even though all adhesive tapes used in the experiment are hypoallergenic. If you have very sensitive skin, please tell the researchers now. If you do not have such sensitivities, please mark your initials here: ___ . Additionally, due to the repetitive nature of the tasks, you may experience slight, discomfort of the hands and/or wrists for a period of up to approximately a day following the trial.

BENEFITS

This study will provide no direct benefits to you, the subject, but it will contribute to the ergonomic tool-design body of knowledge.

\section{CONFIDENTIALITY}

The information in the study records will be kept strictly confidential. Data will be stored securely and will be made available only to persons conducting the study unless you specifically give permission in writing to do otherwise. No reference will be made in oral or written reports that could link you to the study. All videotapes will be destroyed upon completion of data analysis.

\section{COMPENSATION}

For participating in this study you will receive an "Industrial Engineering - Ergonomics" T-shirt. If you withdraw from the study prior to its completion, you will still receive the T-shirt.

EMERGENCY MEDICAL TREATMENT (if applicable)

No compensation for medical treatment will be provided in the event of an injury or illness.

CONTACT

If you have questions at any time about the study or the procedures, you may contact the researcher, Kelly Duke, at 328

Riddick Labs, or 515-7210. If you feel you have not been treated according to the descriptions in this form, or your rights as a participant in research have been violated during the course of this project, you may contact Dr. Matthew Zingraff, Chair of the NCSU IRB for the Use of Human Subjects in Research Committee, Box 7514, NCSU Campus (919/513-1834) or Mr.

Matthew Ronning, Assistant Vice Chancellor, Research Administration, Box 7514, NCSU Campus (919/513-2148)

\section{PARTICIPATION}

Your participation in this study is voluntary; you may decline to participate without penalty. If you decide to participate, you may withdraw from the study at any time without penalty and without loss of benefits to which you are otherwise entitled. If you withdraw from the study before data collection is completed your data will be returned to you or destroyed.

\section{CONSENT}

I have read and understand the above information. I have received a copy of this form. I agree to participate in this study.

Subject's signature

Investigator's signature
Date

Date 